\title{
SPECTRAL ENERGY DISTRIBUTIONS OF LOCAL LUMINOUS AND ULTRALUMINOUS INFRARED GALAXIES
}

\author{
Vivian U ${ }^{1,2,14,15}$, D. B. Sanders ${ }^{1}$, J. M. Mazzarella ${ }^{3}$, A. S. Evans ${ }^{4,5}$, J. H. Howell ${ }^{3,6}$, J. A. Surace ${ }^{6}$, L. Armus ${ }^{6}$, \\ K. Iwasawa ${ }^{7}$, D.-C. $\mathrm{Kim}^{4,5}$, C. M. Casey ${ }^{1}$, T. Vavilkin ${ }^{8}$, M. Dufault ${ }^{9}$, K. L. Larson ${ }^{1}$, J. E. Barnes ${ }^{1}$, B. H. P. Chan ${ }^{3}$, \\ D. T. Frayer ${ }^{10}$, S. HaAN $^{11}$, H. Inami ${ }^{12}$, C. M. Ishida ${ }^{1}$, J. S. Kartaltepe ${ }^{12}$, J. L. Melbourne ${ }^{13}$, And A. O. Petric ${ }^{6}$ \\ ${ }^{1}$ Institute for Astronomy, University of Hawaii, 2680 Woodlawn Drive, Honolulu, HI 96822, USA; vivian@ ifa.hawaii.edu \\ ${ }_{2}^{2}$ Harvard-Smithsonian Center for Astrophysics, Cambridge, MA 02138, USA \\ ${ }^{3}$ Infrared Processing and Analysis Center, California Institute of Technology, 1200 E. California Blvd., Pasadena, CA 91125, USA \\ ${ }^{4}$ Department of Astronomy, University of Virginia, 530 McCormick Road, Charlottesville, VA 22904, USA \\ ${ }^{5}$ National Radio Astronomy Observatory, 520 Edgemont Road, Charlottesville, VA 22903, USA \\ ${ }^{6}$ Spitzer Science Center, 1200 E. California Blvd., MS 314-6, California Institute of Technology, Pasadena, CA 91125, USA \\ ${ }^{7}$ ICREA and Institut del Ciències del Cosmos, Universitat de Barcelona (IEEC-UB), Martí i Franquès, 1, E-08028 Barcelona, Spain \\ ${ }^{8}$ Department of Physics and Astronomy, Stony Brook University, Stony Brook, NY 11794, USA \\ ${ }^{9}$ Department of Astronomy, Yale University, P.O. Box 208101, New Haven, CT 06511, USA \\ ${ }^{10}$ National Radio Astronomy Observatory, P.O. Box 2, Green Bank, WV 24944, USA \\ ${ }^{11}$ CSIRO Astronomy and Space Science, Marsfield NSW, 2122, Australia \\ 12 National Optical Astronomy Observatory, 950 N. Cherry Ave., Tucson, AZ 85719, USA \\ ${ }^{13}$ Caltech Optical Observatories, Division of Physics, Mathematics and Astronomy, MS 320-47, California Institute of Technology, Pasadena, CA 91125, USA \\ Received 2011 October 1; accepted 2012 September 2; published 2012 November 1
}

\begin{abstract}
Luminous (LIRGs; $\left.\log \left(L_{\mathrm{IR}} / L_{\odot}\right)=11.00-11.99\right)$ and ultraluminous infrared galaxies $\left(\mathrm{ULIRGs} ; \log \left(L_{\mathrm{IR}} / L_{\odot}\right)=\right.$ 12.00-12.99) are the most extreme star-forming galaxies in the universe. The local (U)LIRGs provide a unique opportunity to study their multi-wavelength properties in detail for comparison with their more numerous counterparts at high redshifts. We present common large aperture photometry at radio through X-ray wavelengths and spectral energy distributions (SEDs) for a sample of 53 nearby $(z<0.083)$ LIRGs and 11 ULIRGs spanning log $\left(L_{\mathrm{IR}} / L_{\odot}\right)=11.14-12.57$ from the flux-limited $\left(f_{60 \mu \mathrm{m}}>5.24 \mathrm{Jy}\right)$ Great Observatories All-sky LIRG Survey. The SEDs for all objects are similar in that they show a broad, thermal stellar peak $(\sim 0.3-2 \mu \mathrm{m})$, and a dominant FIR $(\sim 40-200 \mu \mathrm{m})$ thermal dust peak, where $v L_{v}(60 \mu \mathrm{m}) / \nu L_{v}(V)$ increases from $\sim 2$ to 30 with increasing $L_{\mathrm{IR}}$. When normalized at IRAS $60 \mu \mathrm{m}$, the largest range in the luminosity ratio, $R(\lambda) \equiv \log \left[\nu L_{\nu}(\lambda) / \nu L_{v}(60 \mu \mathrm{m})\right]$, observed over the full sample is seen in the hard X-rays $(\mathrm{HX}=2-10 \mathrm{keV})$, where $\Delta R_{\mathrm{HX}}=3.73\left(\bar{R}_{\mathrm{HX}}=-3.10\right)$. A small range is found in the radio $(1.4 \mathrm{GHz}), \Delta R_{1.4 \mathrm{GHz}}=1.75$, where the mean ratio is largest, $\left(\bar{R}_{1.4 \mathrm{GHz}}=-5.81\right)$. Total infrared luminosities, $L_{\mathrm{IR}}(8-1000 \mu \mathrm{m})$, dust temperatures, and dust masses were computed from fitting thermal dust emission modified blackbodies to the mid-infrared (MIR) through submillimeter SEDs. The new results reflect an overall $\sim 0.02$ dex lower luminosity than the original IRAS values. Total stellar masses were computed by fitting stellar population synthesis models to the observed near-infrared (NIR) through ultraviolet (UV) SEDs. Mean stellar masses are found to be $\log \left(M_{\star} / M_{\odot}\right)=10.79 \pm 0.40$. Star formation rates have been determined from the infrared $\left(\mathrm{SFR}_{\mathrm{IR}} \sim 45 M_{\odot} \mathrm{yr}^{-1}\right)$ and from the monochromatic UV luminosities $\left(\mathrm{SFR}_{\mathrm{UV}} \sim 1.3 M_{\odot} \mathrm{yr}^{-1}\right)$, respectively. Multi-wavelength active galactic nucleus (AGN) indicators have be used to select putative AGNs: About $60 \%$ of the ULIRGs would have been classified as an AGN by at least one of the selection criteria.
\end{abstract}

Key words: galaxies: active - galaxies: interactions - galaxies: photometry - infrared: galaxies

\section{INTRODUCTION}

Luminous infrared galaxies (LIRGs; $L_{\mathrm{IR}}[8-1000 \mu \mathrm{m}] \geqslant$ $\left.10^{11} L_{\odot}\right)$ are an important class of extragalactic objects. Although relatively rare in the local universe, they still outnumber optically selected starburst and Seyfert galaxies at comparable bolometric luminosity (Soifer et al. 1987), and at the highest luminosities, ultraluminous infrared galaxies, (ULIRGs; $L_{\text {IR }}[8-1000 \mu \mathrm{m}] \geqslant 10^{12} L_{\odot}$ ), exceed the space density of optically selected quasars by a factor of $\sim 3$ (Sanders et al. 1988). Extensive follow-up observations at radio through X-ray wavelengths of complete samples of objects first discovered in the IRAS All-Sky survey, show that (U)LIRGs appear to be powered by a mixture of starburst and active galactic nucleus (AGN) activity, triggered by strong interactions and mergers of gas-

\footnotetext{
${ }^{14}$ NASA Jenkins Predoctoral Fellow.

${ }^{15}$ SAO Predoctoral Fellow.
}

rich spirals (see Sanders \& Mirabel 1996, for a more complete review).

Despite the nearly two decades since the publication of the first IRAS catalogs of (U)LIRGs, there is surprisingly little published photometry that can be used to construct accurate spectral energy distributions (SEDs) for even the nearest and best-studied sources. The majority of the nearby (U)LIRGs are often "messy" systems that do not lend themselves to simple single aperture measurements. Published data not only suffer from different apertures used at both the same and different wavelengths, but also from inconsistent definitions of the true extent and shape of the interacting galaxies, which are often characterized by highly irregular tidal debris fields. The Great Observatories All-sky LIRG Survey (GOALS; Armus et al. 2009) has made one of its top priorities the compilation of a consistent set of photometric images of all LIRGs in the IRAS Revised Bright Galaxy Sample (RBGS; Sanders et al. 2003) by 
reanalyzing existing archival data and obtaining new images at radio through $\mathrm{X}$-ray wavelengths. ${ }^{16}$

This paper presents photometric radio through X-ray SEDs for 64 of the nearest and best-studied (U)LIRGs, using common aperture "masks" to compute accurate total fluxes (including the extended tidal debris fields) for each source. These SEDs are then used to compute basic properties for each source-the total infrared (IR) luminosity, dust temperature, dust mass, and total stellar mass. We also compare SEDs of individual sources in order to understand the expected variation in spectral shapes and colors as well as AGN diagnostics for the complete sample of (U)LIRGs. Understanding the range of spectral and physical properties for this class of objects is critical before any direct comparison to their high- $z$ counterparts can be made.

The paper is organized as follows. Descriptions of the (U)LIRG sample and of the multi-wavelength data sets are provided in Sections 2 and 3, respectively. The complete SEDs and spectral properties are presented in Section 4. Derived and adopted properties, such as the infrared luminosity, $L_{\mathrm{IR}}(8-1000 \mu \mathrm{m})$, dust temperature, dust mass, and stellar mass, $M_{\star}$, are given in Section 5. Our conclusions are summarized in Section 6. Throughout this paper, we adopt a flat model of the universe with a Hubble constant $H_{0}=70 \mathrm{~km} \mathrm{~s}^{-1} \mathrm{Mpc}^{-1}$, and $\Omega_{\mathrm{m}}=0.28$, and $\Omega_{\Lambda}=0.72$ (Komatsu et al. 2009).

\section{SAMPLE}

Our sample of $64(\mathrm{U}) \mathrm{LIRG}$ represents the nearest and brightest infrared-luminous extragalactic sources observable from the northern hemisphere. It is a complete subset of all objects in the IRAS Bright Galaxy Sample (Soifer et al. 1987), with $L_{\mathrm{IR}}>10^{11.14} L_{\odot}$, originally chosen to satisfy the constraints $|b|>30^{\circ}$ and $\delta>-30^{\circ}$ in order to minimize Galactic extinction and to be observable from Mauna Kea, respectively. ${ }^{17}$ Our subsample represents $30 \%$ of all LIRGs and $50 \%$ of all ULIRGs in GOALS. The median infrared luminosity of our subsample is $\log \left(L_{\mathrm{IR}} / L_{\odot}\right)=11.60$, with a luminosity range $\log \left(L_{\mathrm{IR}} / L_{\odot}\right)=11.14-12.57$. The redshift range is $z=0.012-0.083$, corresponding to a luminosity distance, $D_{L}=50.7-387 \mathrm{Mpc}$, with median $z=0.028$ $\left(D_{L}=119.0 \mathrm{Mpc}\right)$. Basic properties of our subsample of GOALS objects are listed in Table 1.

\section{DATA}

The multi-wavelength images for our targets have been assembled from a variety of sources, including our own extensive archive of ground- and space-based observations of (U)LIRGs, as well as data obtained from various data archives. The sources for the images used in our analysis is detailed in Tables 2 and 3. All images were reprocessed and analyzed using a consistent set of criteria. The procedures used to prepare the photometry masks are described in Section 3.3.

\subsection{Space-based Observations}

Observations of the majority of our targets at X-ray, ultraviolet, and infrared wavelengths were obtained as part of major GOALS observing campaigns. The Chandra-GOALS program

\footnotetext{
16 A complete description of the multi-wavelength data can be found on the GOALS Web site at http://www.goals.ipac.caltech.edu.

17 NGC 1068 would have been a part of this sample. However, its proximity, and thus large angular extent, pose a challenge for the UH $2.2 \mathrm{~m}$ Telescope and its limited field of view to capture the entire galaxy and its full debris field. Therefore, it has been left out of the current sample.
}

(Iwasawa et al. 2011) provides X-ray photometry in the soft $\mathrm{X}$-ray (SX; 0.5-2 KeV) and hard X-ray (HX; 2-10 KeV) bands. The GALEX-GOALS program (Howell et al. 2010) provides observations of the majority of our targets in the far-UV (FUV; $\left.\lambda_{\text {eff }}=0.1528 \mu \mathrm{m}\right)$ and near-UV (NUV; $\lambda_{\text {eff }}=0.2271 \mu \mathrm{m}$ ) bands. The Spitzer-GOALS programs (J. M. Mazzarella et al. 2012, in preparation; J. A. Surace et al. 2012, in preparation) obtained MIR (3.6, 4.6, 5.4, and $8.0 \mu \mathrm{m})$ and FIR (24, 70, and $160 \mu \mathrm{m}$ ) images with IRAC and MIPS, respectively. The Herschel-GOALS programs (PIs: Sanders and Armus) involving PACS and SPIRE observations are currently ongoing and will be completed in late 2012. IRAS data at 12, 25, 60, and $100 \mu \mathrm{m}$ as published in the RBGS (Sanders et al. 2003) have also been incorporated into our analysis for completeness.

\subsection{Ground-based Observations}

Most of the observations in the optical bands were taken with the Tektronix $2048 \times 2048$ camera (hereafter, Tek2048) at the University of Hawaii (UH) $2.2 \mathrm{~m}$ Telescope on Mauna Kea. $B V I$-band images for 53 LIRGs +1 ULIRG were originally obtained as part of a PhD thesis that studied a complete subsample of LIRGs from the IRAS BGS (see Ishida 2004, for details on the observations and reduction). The primary source of the $R$-band images is the RBGS $R$ - $K^{\prime}$ Atlas (J. M. Mazzarella et al. 2012, in preparation), while Surace et al. $(1998,2000)$ and Surace \& Sanders (2000) provide UBI-band data for many of the ULIRGs, all imaged with either the Tek2048 or the Orbit $2048 \times 2048$ cameras on the UH 2.2 m Telescope.

The remaining optical, near-infrared (NIR), submillimeter, and radio data have been compiled from the literature and the NASA/IPAC Extragalactic Database (NED). Most notably, $J H K_{S}$ images were extracted from the Two Micron All Sky Survey (2MASS; Skrutskie et al. 2006) Extended Source Image Server, and in the case of large systems spanning multiple frames, the Large Galaxy Atlas (Jarrett et al. 2003) via the InfraRed Science Archive. Submillimeter data at $850 \mu \mathrm{m}$ and $450 \mu \mathrm{m}$, obtained using the Submillimeter CommonUser Bolometer Array (SCUBA) at the James Clerk Maxwell Telescope were taken from Dunne et al. (2000) and Dunne \& Eales (2001), while $1.49 \mathrm{GHz}$ radio data obtained at the Very Large Array (VLA) were taken from Condon et al. (1990, 1996). All of our archival data sources are summarized in Table 2.

The remaining gaps in our optical photometry table were filled by observed with the Tek2048 camera on the UH $2.2 \mathrm{~m}$ Telescope during two observing runs, totaling eight partially photometric nights in February and August of 2008. Each exposure was typically 2-6 minutes, with seeing ranging between $00^{\prime \prime} .5$ and 0.8 , (see Table 3 for a summary). A dither pattern of $80^{\prime \prime} .0 \times 80^{\prime} .0$ was used during the observations. The data were reduced and calibrated using various packages provided by the IDL astron library. The reduction involved several standard steps: All images were bias subtracted using a median bias computed from bias frames taken at the beginning of each night. There is a location-dependent shutter correction time of $0.18 \mathrm{~s}$ (H. Courtois 2008, private communication) for the Tek2048; this is a significant contribution for the images with short exposure times (calibration stars in particular), so all of the data were corrected by this factor. For each of the UVRI filters during each night of the run, a master flat, created from median-combining normalized flats, was used to flat field all the corresponding science images. After adding astrometry information (using IDL routine STARAST) to the image headers, science images observed close in time within 
Table 1

Local (U)LIRG Sample

\begin{tabular}{|c|c|c|c|c|c|}
\hline RBGS Name & $\begin{array}{c}\text { R.A. } \\
(\mathrm{J} 2000)\end{array}$ & $\begin{array}{c}\text { Decl. } \\
(\mathrm{J} 2000)\end{array}$ & $\begin{array}{c}\log \left(L_{\mathrm{IR}}\right)_{I R A S} \\
\left(L_{\odot}\right)\end{array}$ & $\begin{array}{c}D_{L} \\
(\mathrm{Mpc})\end{array}$ & Other Names \\
\hline NGC 0034 & 001106.5 & -120626 & 11.49 & 84.1 & NGC 0017, VV 860, Mrk 0938 \\
\hline MCG -02-01-051/2 & 001850.4 & -102208 & 11.48 & 117.5 & Arp 256, VV 352 \\
\hline NGC 0232 & 004245.8 & -233341 & 11.44 & 95.2 & VV 830 \\
\hline IC $1623 \mathrm{~A} / \mathrm{B}$ & 010747.2 & -173025 & 11.71 & 85.5 & Arp 236, VV 114 \\
\hline MCG -03-04-014 & 011008.9 & -165110 & 11.65 & 144.0 & \\
\hline CGCG 436-030 & 012002.7 & +142143 & 11.69 & 134.0 & \\
\hline IRAS F01364-1042 & 013852.9 & -102711 & 11.85 & 210.0 & \\
\hline III Zw 035 & 014430.5 & +170605 & 11.64 & 119.0 & \\
\hline NGC 0695 & 015114.2 & +223457 & 11.68 & 139.0 & UGC 01315, V Zw 123 \\
\hline NGC 0958 & 023042.8 & -025620 & 11.20 & 80.6 & \\
\hline UGC 02238 & 024617.5 & +130544 & 11.33 & 92.4 & \\
\hline UGC 02369 & 025401.8 & +145825 & 11.67 & 136.0 & \\
\hline IRAS F03359+1523 & 033846.7 & +153255 & 11.55 & 152.0 & \\
\hline UGC 02982 & 041222.4 & +053251 & 11.20 & 74.9 & \\
\hline ESO 550-IG025 & 042120.0 & -184848 & 11.51 & 138.5 & \\
\hline NGC 1614 & 043359.8 & -083444 & 11.65 & 67.8 & Arp 186, Mrk 0617, II Zw 015 \\
\hline IRAS F05189-2524 & 052101.5 & -252145 & 12.16 & 187.0 & \\
\hline NGC 2623 & 083824.1 & +254517 & 11.60 & 84.1 & UGC 04509, Arp 243, VV 079 \\
\hline IRAS F08572+3915 & 090025.4 & +390354 & 12.16 & 264.0 & \\
\hline UGC 04881 & 091555.1 & +441955 & 11.74 & 178.0 & Arp 055, VV 155 \\
\hline UGC 05101 & 093551.6 & +612111 & 12.01 & 177.0 & \\
\hline $\mathrm{MCG}+08-18-012 / 3$ & 093637.2 & +482828 & 11.34 & 117.0 & CGCG 239-011 \\
\hline IC $0563 / 4$ & 094620.6 & +030330 & 11.23 & 92.9 & UGC 05230, Arp 303 \\
\hline NGC 3110 & 100402.1 & -062829 & 11.37 & 79.5 & NGC 3122, NGC 3518 \\
\hline IRAS F10173+0828 & 102000.2 & +081334 & 11.86 & 224.0 & \\
\hline IRAS F10565+2448 & 105918.1 & +243235 & 12.08 & 197.0 & \\
\hline MCG +07-23-019 & 110353.2 & +405057 & 11.62 & 158.0 & Arp 148, VV 032 \\
\hline CGCG 011-076 & 112112.2 & -025903 & 11.43 & 117.0 & \\
\hline IC $2810 A / B$ & 112545.0 & +144036 & 11.64 & 157.0 & UGC 06436 \\
\hline NGC 3690/IC 694 & 112830.4 & +583410 & 11.93 & 50.7 & Arp 299, VV 118 \\
\hline IRAS F12112+0305 & 121346.0 & +024838 & 12.36 & 340.0 & \\
\hline IRAS F12224-0624 & 122503.9 & -064053 & 11.36 & 125.0 & \\
\hline UGC 08058 & 125614.2 & +565225 & 12.57 & 192.0 & Mrk 0231, VII Zw 490 \\
\hline NGC 4922 & 130124.9 & +291840 & 11.38 & 111.0 & UGC 08135, VV 609 \\
\hline ESO 507-G070 & 130252.3 & -235518 & 11.56 & 106.0 & \\
\hline IC 0860 & 131503.5 & +243708 & 11.14 & 56.8 & \\
\hline VV 250 & 131532.8 & +620737 & 11.81 & 142.0 & UGC 08335, Arp 238, VII Zw 506 \\
\hline UGC 08387 & 132035.3 & +340822 & 11.73 & 110.0 & IC 0883, Arp 193, VV 821, I Zw 056 \\
\hline NGC 5104 & 132123.1 & +002033 & 11.27 & 90.8 & UGC 08391 \\
\hline MCG -03-34-064 & 132224.4 & -164343 & 11.28 & 82.2 & \\
\hline NGC 5135 & 132544.0 & -295001 & 11.30 & 60.9 & \\
\hline NGC 5256 & 133817.5 & +481637 & 11.56 & 129.0 & UGC 08632, Mrk 0266, I Zw 067 \\
\hline NGC 5257/8 & 133955.2 & +005013 & 11.62 & 108.5 & Arp 240, VV 055 \\
\hline UGC 08696 & 134442.1 & +555313 & 12.21 & 173.0 & Mrk 0273, VV 851, I Zw 071 \\
\hline CGCG 247-020 & 141943.2 & +491412 & 11.39 & 120.0 & Mrk 1490 \\
\hline IRAS F14348-1447 & 143738.3 & -150023 & 12.39 & 387.0 & \\
\hline VV 340 & 145700.4 & +243644 & 11.74 & 157.0 & UGC 09618, Arp 302 \\
\hline CGCG 049-057 & 151313.1 & +071332 & 11.35 & 65.4 & \\
\hline VV 705 & 151806.3 & +424437 & 11.92 & 183.0 & Mrk 0848, I Zw 107 \\
\hline IRAS F15250+3608 & 152659.4 & +355838 & 12.08 & 254.0 & \\
\hline UGC 09913 & 153457.1 & +233011 & 12.28 & 87.9 & Arp 220, VV 540 \\
\hline NGC 6090 & 161140.7 & +522724 & 11.58 & 137.0 & UGC 10267, VV 626, Mrk 0496, I Zw 135 \\
\hline CGCG 052-037 & 163056.5 & +040458 & 11.45 & 116.0 & \\
\hline NGC 6285/6 & 165827.8 & +585648 & 11.37 & 85.7 & Arp 293 \\
\hline IRAS F17132+5313 & 171420.0 & +531030 & 11.96 & 232.0 & \\
\hline ESO 602-G025 & 223125.5 & -190204 & 11.34 & 110.0 & \\
\hline IRAS F22491-1808 & 225149.2 & -175223 & 12.20 & 351.0 & \\
\hline NGC 7469/IC 5283 & 230316.9 & +085301 & 11.65 & 70.8 & Arp 298, UGC 12332 \\
\hline CGCG 453-062 & 230456.5 & +193308 & 11.38 & 109.0 & \\
\hline IC 5298 & 231600.7 & +253324 & 11.60 & 119.0 & \\
\hline NGC 7592 & 231822.2 & +042501 & 11.40 & 106.0 & VV 731 \\
\hline NGC 7674 & 232757.8 & +084651 & 11.56 & 125.0 & UGC 12608, Arp 182, VV 343, Mrk 0533 \\
\hline NGC $7770 / 1$ & 235124.9 & +200643 & 11.40 & 61.2 & UGC 12815, Mrk 9006 \\
\hline MRK 0331 & 235126.8 & +203510 & 11.50 & 70.5 & \\
\hline
\end{tabular}

Note. The $L_{\mathrm{IR}}$ column indicates infrared luminosities based on IRAS measurements only. 
Table 2

Major Data Sets

\begin{tabular}{|c|c|c|c|}
\hline Telescope & No. of Objects & Bands & Reference \\
\hline Chandra & 22 & $0.5-2 \mathrm{keV}, 2-10 \mathrm{keV}$ & Iwasawa et al. (2011) \\
\hline$G A L E X$ & 57 & NUV, FUV & Howell et al. (2010) \\
\hline $\mathrm{UH} 2.2 \mathrm{~m}$ & 64 & $B, V, I$ & Ishida (2004) \\
\hline $\mathrm{UH} 2.2 \mathrm{~m}$ & 8 & $U, B$ & Surace et al. (1998, 2000); Surace \& Sanders (2000) \\
\hline $\mathrm{UH} 2.2 \mathrm{~m}$ & 46 & $R$ & J. M. Mazzarella et al. (2012, in preparation) \\
\hline 2MASS & 64 & $J, H, K_{\mathrm{s}}$ & Skrutskie et al. (2006); Jarrett et al. (2003) \\
\hline Spitzer & 64 & $3.6,4.5,5.8,8.0,24,70,160 \mu \mathrm{m}$ & J. M. Mazzarella et al. (2012, in preparation); J. A. Surace et al. (2012, in preparation) \\
\hline IRAS & 64 & $12,25,60,100 \mu \mathrm{m}$ & Sanders et al. (2003) \\
\hline JCMT/SCUBA & 42 & $450,850 \mu \mathrm{m}$ & Dunne et al. (2000); Dunne \& Eales (2001) \\
\hline VLA & 64 & $1.49,4.85 \mathrm{GHz}$ & Condon et al. (1990, 1996) \\
\hline
\end{tabular}

Table 3

New UH 2.2 m Observations

\begin{tabular}{|c|c|c|c|c|}
\hline RBGS Name & $\begin{array}{l}\text { Exp. Time } \\
\text { (s) }\end{array}$ & $\begin{array}{c}\text { Filter } \\
\text { (Johnson) }\end{array}$ & $\begin{array}{l}\text { Seeing } \\
(\operatorname{arcsec})\end{array}$ & $\begin{array}{l}\text { Obs. Date } \\
\text { (UT) }\end{array}$ \\
\hline CGCG 436-030 & 180 & $R$ & 0.7 & 2008 Sep 1 \\
\hline IRAS F01364-1042 & 360 & $U$ & 0.6 & 2008 Aug 28 \\
\hline NGC 0695 & 180 & $R$ & 0.7 & 2008 Sep 1 \\
\hline \multirow[t]{2}{*}{ IRAS F05189-2524 } & 180 & $V$ & 0.6 & 2008 Sep 1 \\
\hline & 180 & $I$ & 0.6 & 2008 Sep 1 \\
\hline IRAS F08572+3915 & 180 & $V$ & 0.7 & 2008 Feb 15 \\
\hline \multirow[t]{2}{*}{ IRAS F10565+2448 } & 330 & $U$ & 0.6 & 2008 Feb 18 and 19 \\
\hline & 180 & $V$ & 0.7 & 2008 Feb 18 \\
\hline IRAS F12112+0305 & 180 & $V$ & 0.5 & 2008 Feb 19 \\
\hline UGC 08058 & 120 & $I$ & 0.8 & 2008 Feb 16 \\
\hline UGC 08696 & 120 & $V$ & 0.5 & 2008 Feb 17 \\
\hline IRAS F14348-1447 & 180 & $V$ & 0.7 & 2008 Feb 15 \\
\hline IRAS F22491-1808 & 180 & $V$ & 0.7 & 2008 Aug 28 \\
\hline
\end{tabular}

the same night were co-added to increase the signal-to-noise ratio for each target frame. The data were subsequently calibrated with standard stars in the corresponding optical bands (Landolt 1983), taking into account airmass corrections. To ensure that consistent apertures were used to find the total flux from each galaxy system, photometry was subsequently measured using previously constructed "masks," as described in Section 3.3.

\subsection{Photometry Masks}

When assembling SEDs for our sources, we constructed a single photometric mask for each source that was designed to incorporate the total flux from a galaxy system whether it consists of a single galaxy with disturbed morphology or two separate interacting galaxies. The masks have been defined based on isophotes in the median- and boxcar-smoothed I-band images at the surface brightness limit of $24.5 \mathrm{mag} \operatorname{arcsec}^{-2}$ (Vavilkin et al. 2011). They are intended to encapsulate the global flux from tidal debris as well as individual components within these merger systems. The more luminous 43 objects in our sample $\left(L_{\mathrm{IR}}>11.4\right)$ have masks defined using HST-ACS images (A. S. Evans et al. 2012, in preparation); the other 21 masks were generated using the same technique from groundbased $I$-band images taken with the UH $2.2 \mathrm{~m}$ Telescope. We have tested for systematic bias between the masks made from Hubble Space Telescope (HST) and ground-based images and found that the difference in measured photometry is less than $0.2 \%$, within photometric uncertainties. The photometry masks for all 64 (U)LIRGs are shown in Figure 1.

Masked photometry has been extracted from images taken at effective wavelength $0.15 \mu \mathrm{m}<\lambda_{\text {eff }}<8 \mu \mathrm{m}$ and at MIPS
$24 \mu \mathrm{m}$ band. The short wavelength limit has been imposed due to the fact that the X-ray photon counts are fairly localized, whereas on the longward side, the images are lacking in resolution ( $\gtrsim$ '.0 point spread function). At either end of the wavelength range, therefore, masks would not improve the precision of the total flux measurement over that of circular or elliptical apertures. Differential emission will be taken into account when deep optical/NIR images probing the extended debris field are acquired in the near future (Spitzer snapshot program; PI: Sanders). Within the wavelength regime where masks have been applied, the masks are very large relative to the beam size and hence aperture corrections are not needed.

\section{SPECTRAL ENERGY DISTRIBUTION OF THE GOALS SAMPLE}

In this section, we present SEDs (radio through X-ray) for our complete sample of 64 (U)LIRGs. The common aperture mask photometry data are provided in Table 4 (X-ray to NIR) and Table 5 (MIR to radio). The complete radio through X-ray SEDs $\left(\log v L_{v}\right.$ versus $\left.\log v\right)$ for each source are shown in the panels of Figure 2. The photometric data points are overlaid with the best-fit model SEDs, which will be discussed in more detail in Section 5.1.

(U)LIRGs are known to exhibit several common features in their SEDs, e.g., a drop-off in the UV flux, an optical-NIR stellar thermal "bump," and a thermal dust "bump" at FIR wavelengths (Sanders \& Mirabel 1996). However, photometry compiled for our complete local sample displays varied spectral shapes across the electromagnetic spectrum. The main goal of our current study is to characterize the full SED and spectral properties of (U)LIRGs as follows.

\subsection{Spectral Shapes}

Before we employ the SEDs of these (U)LIRGs as local templates, we must first understand the variations in the spectral shapes across the range of various attributes. In particular, Figure 3 shows the radio through X-ray SEDs of all 64 (U)LIRGs. Normalized at $J$ band, this plot shows the bestfit modeled fluxes at the observed filters and brings out the variations in the optical (UV-NIR) regime, the shortward end of which at FUV is characterized by the young stellar populations in the galaxies. No attempt has been made to connect the observed X-ray emission to the UV flux given our lack of data in the nearly two decade wavelength range between the FUV and SX measurements.

To better demonstrate both the range and mean of the object SEDs, we show in Figure 4 the mean SED along with the 25\%, $75 \%$, and $\mathrm{max} / \mathrm{min}$ values for the 11 ULIRGs and 53 LIRGs 
Table 4

Local (U)LIRG Photometry (Jy; $\lambda<3 \mu \mathrm{m}$ )

\begin{tabular}{|c|c|c|c|c|c|c|c|c|c|c|c|c|}
\hline RBGS Name & $\begin{array}{c}\mathrm{HX} \\
2-10 \mathrm{KeV} \\
(2)\end{array}$ & $\begin{array}{c}\text { SX } \\
0.5-2 \mathrm{KeV} \\
(3)\end{array}$ & $\begin{array}{c}\text { FUV } \\
152.8 \AA \\
(4)\end{array}$ & $\begin{array}{c}\text { NUV } \\
227.1 \AA \\
(5)\end{array}$ & $\begin{array}{c}U \\
3655 \AA \\
(6)\end{array}$ & $\begin{array}{c}B \\
4582 \AA \\
(7)\end{array}$ & $\begin{array}{c}V \\
5377 \AA \\
(8)\end{array}$ & $\begin{array}{c}R \\
6484 \AA \\
(9)\end{array}$ & $\begin{array}{c}I \\
8570 \AA \\
(10)\end{array}$ & $\begin{array}{c}J \\
1.241 \mu \mathrm{m} \\
(11)\end{array}$ & $\begin{array}{c}H \\
1.651 \mu \mathrm{m} \\
(12)\end{array}$ & $\begin{array}{c}K_{\mathrm{S}} \\
2.165 \mu \mathrm{m} \\
(13)\end{array}$ \\
\hline NGC 0034 & $\begin{array}{c}1.586 \mathrm{E}-08^{\mathrm{a}} \\
(\ldots)\end{array}$ & $\begin{array}{c}2.318 \mathrm{E}-08^{\mathrm{a}} \\
(\ldots)\end{array}$ & $\begin{array}{c}5.249 \mathrm{E}-04 \\
(6.480 \mathrm{E}-05)\end{array}$ & $\begin{array}{c}1.262 \mathrm{E}-03 \\
(7.126 \mathrm{E}-05)\end{array}$ & $(\ldots)$ & $\begin{array}{c}1.076 \mathrm{E}-02 \\
(5.949 \mathrm{E}-04)\end{array}$ & $\begin{array}{c}1.629 \mathrm{E}-02 \\
(9.004 \mathrm{E}-04)\end{array}$ & $\begin{array}{c}1.740 \mathrm{E}-02 \\
(1.122 \mathrm{E}-03)\end{array}$ & $\begin{array}{c}2.679 \mathrm{E}-02 \\
(1.481 \mathrm{E}-03)\end{array}$ & $\begin{array}{c}5.225 \mathrm{E}-02 \\
(1.357 \mathrm{E}-04)\end{array}$ & $\begin{array}{c}6.529 \mathrm{E}-02 \\
(2.097 \mathrm{E}-04)\end{array}$ & $\begin{array}{c}6.557 \mathrm{E}-02 \\
(1.783 \mathrm{E}-04)\end{array}$ \\
\hline MCG -02-01-051/2 & $\begin{array}{l}\ldots \\
(\ldots)\end{array}$ & $\begin{array}{l}\ldots \\
(\ldots)\end{array}$ & $\begin{array}{c}2.255 \mathrm{E}-03 \\
(2.784 \mathrm{E}-04)\end{array}$ & $\begin{array}{c}3.279 \mathrm{E}-03 \\
(1.851 \mathrm{E}-04)\end{array}$ & $\begin{array}{l}\ldots \\
(\ldots)\end{array}$ & $\begin{array}{c}1.028 \mathrm{E}-02 \\
(1.136 \mathrm{E}-03)\end{array}$ & $\begin{array}{c}1.368 \mathrm{E}-02 \\
(1.512 \mathrm{E}-03)\end{array}$ & $\begin{array}{l}\ldots \\
(\ldots)\end{array}$ & $\begin{array}{c}2.032 \mathrm{E}-02 \\
(2.246 \mathrm{E}-03)\end{array}$ & $\begin{array}{c}3.552 \mathrm{E}-02 \\
(1.287 \mathrm{E}-04)\end{array}$ & $\begin{array}{c}3.808 \mathrm{E}-02 \\
(3.044 \mathrm{E}-04)\end{array}$ & $\begin{array}{c}3.247 \mathrm{E}-02 \\
(2.060 \mathrm{E}-04)\end{array}$ \\
\hline NGC 0232 & $\begin{array}{l}\ldots \\
(\ldots)\end{array}$ & $\begin{array}{l}\ldots \\
(\ldots)\end{array}$ & $\begin{array}{c}2.038 \mathrm{E}-04 \\
(1.538 \mathrm{E}-05)\end{array}$ & $\begin{array}{c}3.880 \mathrm{E}-04 \\
(1.637 \mathrm{E}-05)\end{array}$ & $\begin{array}{l}\ldots \\
(\ldots)\end{array}$ & $\begin{array}{c}1.535 \mathrm{E}-02 \\
(2.097 \mathrm{E}-03)\end{array}$ & $\begin{array}{c}3.071 \mathrm{E}-02 \\
(2.220 \mathrm{E}-03)\end{array}$ & $\begin{array}{c}1.461 \mathrm{E}-02 \\
(9.417 \mathrm{E}-04)\end{array}$ & $\begin{array}{c}6.925 \mathrm{E}-02 \\
(1.148 \mathrm{E}-02)\end{array}$ & $\begin{array}{c}6.128 \mathrm{E}-02 \\
(9.594 \mathrm{E}-04)\end{array}$ & $\begin{array}{c}8.037 \mathrm{E}-02 \\
(1.480 \mathrm{E}-03)\end{array}$ & $\begin{array}{c}\text { 7.487E-02 } \\
(1.448 \mathrm{E}-03)\end{array}$ \\
\hline IC $1623 \mathrm{~A} / \mathrm{B}$ & $\begin{array}{l}\ldots \\
(\ldots)\end{array}$ & $\begin{array}{l}\ldots \\
(\ldots)\end{array}$ & $\begin{array}{c}5.624 \mathrm{E}-03 \\
(6.942 \mathrm{E}-04)\end{array}$ & $\begin{array}{c}7.898 \mathrm{E}-03 \\
(4.459 \mathrm{E}-04)\end{array}$ & $\begin{array}{l}\ldots \\
(\ldots)\end{array}$ & $\begin{array}{c}1.871 \mathrm{E}-02 \\
(1.034 \mathrm{E}-03)\end{array}$ & $\begin{array}{c}2.489 \mathrm{E}-02 \\
(1.375 \mathrm{E}-03)\end{array}$ & $\begin{array}{c}3.569 \mathrm{E}-02 \\
(2.301 \mathrm{E}-03)\end{array}$ & $\begin{array}{c}3.467 \mathrm{E}-02 \\
(1.916 \mathrm{E}-03)\end{array}$ & $\begin{array}{c}6.292 \mathrm{E}-02 \\
(1.304 \mathrm{E}-04)\end{array}$ & $\begin{array}{c}7.755 \mathrm{E}-02 \\
(1.906 \mathrm{E}-04)\end{array}$ & $\begin{array}{c}7.716 \mathrm{E}-02 \\
(1.958 \mathrm{E}-04)\end{array}$ \\
\hline MCG -03-04-014 & $\begin{array}{l}\ldots \\
(\ldots)\end{array}$ & $\begin{array}{l}\ldots \\
(\ldots)\end{array}$ & $\begin{array}{c}2.402 \mathrm{E}-04 \\
(2.965 \mathrm{E}-05)\end{array}$ & $\begin{array}{c}5.692 \mathrm{E}-04 \\
(3.213 \mathrm{E}-05)\end{array}$ & $(\ldots)$ & $\begin{array}{c}4.093 \mathrm{E}-03 \\
(2.639 \mathrm{E}-04)\end{array}$ & $\begin{array}{c}6.792 \mathrm{E}-03 \\
(4.379 \mathrm{E}-04)\end{array}$ & $\begin{array}{c}8.801 \mathrm{E}-03 \\
(5.674 \mathrm{E}-04)\end{array}$ & $\begin{array}{c}1.169 \mathrm{E}-02 \\
(6.463 \mathrm{E}-04)\end{array}$ & $\begin{array}{c}3.336 \mathrm{E}-02 \\
(1.085 \mathrm{E}-04)\end{array}$ & $\begin{array}{c}4.045 \mathrm{E}-02 \\
(1.456 \mathrm{E}-04)\end{array}$ & $\begin{array}{c}3.829 \mathrm{E}-02 \\
(1.494 \mathrm{E}-04)\end{array}$ \\
\hline CGCG 436-030 & $\begin{array}{l}\ldots \\
(\ldots)\end{array}$ & $\begin{array}{l}\ldots \\
(\ldots)\end{array}$ & $\begin{array}{c}4.304 \mathrm{E}-04 \\
(5.313 \mathrm{E}-05)\end{array}$ & $\begin{array}{c}7.831 \mathrm{E}-04 \\
(4.421 \mathrm{E}-05)\end{array}$ & $\begin{array}{l}\ldots \\
(\ldots)\end{array}$ & $\begin{array}{c}4.130 \mathrm{E}-03 \\
(3.424 \mathrm{E}-04)\end{array}$ & $\begin{array}{c}6.310 \mathrm{E}-03 \\
(4.649 \mathrm{E}-04)\end{array}$ & $\begin{array}{c}8.075 \mathrm{E}-03 \\
(1.487 \mathrm{E}-03)\end{array}$ & $\begin{array}{c}1.019 \mathrm{E}-02 \\
(2.064 \mathrm{E}-03)\end{array}$ & $\begin{array}{c}1.702 \mathrm{E}-02 \\
(9.198 \mathrm{E}-05)\end{array}$ & $\begin{array}{c}2.365 \mathrm{E}-02 \\
(1.101 \mathrm{E}-04)\end{array}$ & $\begin{array}{c}2.286 \mathrm{E}-02 \\
(1.610 \mathrm{E}-04)\end{array}$ \\
\hline IRAS F01364-1042 & $\begin{array}{c}1.961 \mathrm{E}-09 \\
(\ldots)\end{array}$ & $\begin{array}{c}1.506 \mathrm{E}-09 \\
(\ldots)\end{array}$ & $\begin{array}{c}2.456 \mathrm{E}-05 \\
(2.738 \mathrm{E}-06)\end{array}$ & $\begin{array}{c}5.314 \mathrm{E}-05 \\
(2.811 \mathrm{E}-06)\end{array}$ & $\begin{array}{c}4.786 \mathrm{E}-05 \\
(2.047 \mathrm{E}-05)\end{array}$ & $\begin{array}{c}5.989 \mathrm{E}-04 \\
(7.347 \mathrm{E}-05)\end{array}$ & $\begin{array}{c}1.223 \mathrm{E}-03 \\
(8.546 \mathrm{E}-05)\end{array}$ & $\begin{array}{l}9.056 \mathrm{E}-04 \\
(5.839 \mathrm{E}-05)\end{array}$ & $\begin{array}{c}2.239 \mathrm{E}-03 \\
(3.013 \mathrm{E}-04)\end{array}$ & $\begin{array}{c}6.269 \mathrm{E}-03 \\
(5.634 \mathrm{E}-05)\end{array}$ & $\begin{array}{c}7.249 \mathrm{E}-03 \\
(7.102 \mathrm{E}-05)\end{array}$ & $\begin{array}{c}8.223 \mathrm{E}-03 \\
(7.910 \mathrm{E}-05)\end{array}$ \\
\hline III Zw 035 & $\begin{array}{l}\ldots \\
(\ldots)\end{array}$ & $\begin{array}{l}\ldots \\
(\ldots)\end{array}$ & $\begin{array}{c}5.246 \mathrm{E}-05 \\
(6.476 \mathrm{E}-06)\end{array}$ & $\begin{array}{c}1.132 \mathrm{E}-04 \\
(6.390 \mathrm{E}-06)\end{array}$ & $\begin{array}{l}\ldots \\
(\ldots)\end{array}$ & $\begin{array}{c}1.570 \mathrm{E}-03 \\
(1.012 \mathrm{E}-04)\end{array}$ & $\begin{array}{c}2.911 \mathrm{E}-03 \\
(1.877 \mathrm{E}-04)\end{array}$ & $\begin{array}{c}2.111 \mathrm{E}-03 \\
(1.361 \mathrm{E}-04)\end{array}$ & $\begin{array}{c}5.495 \mathrm{E}-03 \\
(6.074 \mathrm{E}-04)\end{array}$ & $\begin{array}{c}1.176 \mathrm{E}-02 \\
(5.151 \mathrm{E}-05)\end{array}$ & $\begin{array}{c}1.484 \mathrm{E}-02 \\
(6.033 \mathrm{E}-05)\end{array}$ & $\begin{array}{c}1.485 \mathrm{E}-02 \\
(6.564 \mathrm{E}-05)\end{array}$ \\
\hline NGC 0695 & $\begin{array}{l}\ldots \\
(\ldots)\end{array}$ & $\begin{array}{l}\ldots \\
(\ldots)\end{array}$ & $\begin{array}{c}3.526 \mathrm{E}-04 \\
(4.352 \mathrm{E}-05)\end{array}$ & $\begin{array}{c}7.967 \mathrm{E}-04 \\
(4.498 \mathrm{E}-05)\end{array}$ & $\begin{array}{l}\ldots \\
(\ldots)\end{array}$ & $\begin{array}{c}7.727 \mathrm{E}-03 \\
(7.828 \mathrm{E}-04)\end{array}$ & $\begin{array}{c}1.343 \mathrm{E}-02 \\
(3.710 \mathrm{E}-04)\end{array}$ & $\begin{array}{c}1.830 \mathrm{E}-02 \\
(3.370 \mathrm{E}-03)\end{array}$ & $\begin{array}{c}2.655 \mathrm{E}-02 \\
(7.335 \mathrm{E}-04)\end{array}$ & $\begin{array}{c}4.630 \mathrm{E}-02 \\
(1.001 \mathrm{E}-04)\end{array}$ & $\begin{array}{c}5.962 \mathrm{E}-02 \\
(1.188 \mathrm{E}-04)\end{array}$ & $\begin{array}{c}5.437 \mathrm{E}-02 \\
(1.226 \mathrm{E}-04)\end{array}$ \\
\hline NGC 0958 & $\begin{array}{l}\ldots \\
(\ldots)\end{array}$ & $\begin{array}{l}\ldots \\
(\ldots)\end{array}$ & $\begin{array}{l}1.084 \mathrm{E}-03^{\mathrm{a}} \\
(7.689 \mathrm{E}-06)\end{array}$ & $\begin{array}{c}2.079 \mathrm{E}-03 \\
(6.549 \mathrm{E}-06)\end{array}$ & $\begin{array}{c}1.959 \mathrm{E}-02 \\
(2.526 \mathrm{E}-03)\end{array}$ & $\begin{array}{c}2.128 \mathrm{E}-02 \\
(1.176 \mathrm{E}-03)\end{array}$ & $\begin{array}{c}3.837 \mathrm{E}-02 \\
(2.120 \mathrm{E}-03)\end{array}$ & $\begin{array}{l}\ldots \\
(\ldots)\end{array}$ & $\begin{array}{c}8.318 \mathrm{E}-02 \\
(3.830 \mathrm{E}-03)\end{array}$ & $\begin{array}{c}1.798 \mathrm{E}-01 \\
(1.988 \mathrm{E}-03)\end{array}$ & $\begin{array}{c}2.335 \mathrm{E}-01 \\
(3.226 \mathrm{E}-03)\end{array}$ & $\begin{array}{c}2.013 \mathrm{E}-01 \\
(3.709 \mathrm{E}-03)\end{array}$ \\
\hline UGC 02238 & $\begin{array}{l}\ldots \\
(\ldots)\end{array}$ & $\begin{array}{l}\ldots \\
(\ldots)\end{array}$ & $\begin{array}{c}1.092 \mathrm{E}-04 \\
(5.382 \mathrm{E}-06)\end{array}$ & $\begin{array}{c}2.096 \mathrm{E}-04 \\
(4.912 \mathrm{E}-06)\end{array}$ & $(\ldots)$ & $\begin{array}{c}3.873 \mathrm{E}-03 \\
(1.427 \mathrm{E}-04)\end{array}$ & $\begin{array}{c}7.311 \mathrm{E}-03 \\
(2.020 \mathrm{E}-04)\end{array}$ & $(\ldots)$ & $\begin{array}{c}1.660 \mathrm{E}-02 \\
(4.586 \mathrm{E}-04)\end{array}$ & $\begin{array}{c}3.430 \mathrm{E}-02 \\
(8.845 \mathrm{E}-04)\end{array}$ & $\begin{array}{c}4.897 \mathrm{E}-02 \\
(1.037 \mathrm{E}-03)\end{array}$ & $\begin{array}{c}5.267 \mathrm{E}-02 \\
(1.407 \mathrm{E}-03)\end{array}$ \\
\hline UGC 02369 & $\begin{array}{l}\ldots \\
(\ldots)\end{array}$ & $\begin{array}{l}\ldots \\
(\ldots)\end{array}$ & $\begin{array}{l}\ldots \\
(\ldots)\end{array}$ & $\begin{array}{l}\ldots \\
(\ldots)\end{array}$ & $\begin{array}{l}\ldots \\
(\ldots)\end{array}$ & $\begin{array}{c}5.058 \mathrm{E}-03 \\
(1.398 \mathrm{E}-04)\end{array}$ & $\begin{array}{c}1.138 \mathrm{E}-02 \\
(2.096 \mathrm{E}-04)\end{array}$ & $\begin{array}{c}1.344 \mathrm{E}-02 \\
(8.668 \mathrm{E}-04)\end{array}$ & $\begin{array}{c}2.704 \mathrm{E}-02 \\
(1.245 \mathrm{E}-03)\end{array}$ & $\begin{array}{c}5.274 \mathrm{E}-02 \\
(1.330 \mathrm{E}-04)\end{array}$ & $\begin{array}{c}6.806 \mathrm{E}-02 \\
(2.199 \mathrm{E}-04)\end{array}$ & $\begin{array}{c}6.325 \mathrm{E}-02 \\
(1.806 \mathrm{E}-04)\end{array}$ \\
\hline IRAS F03359+1523 & $\begin{array}{l}\ldots \\
(\ldots)\end{array}$ & $\begin{array}{l}\ldots \\
(\ldots)\end{array}$ & $\begin{array}{c}1.054 \mathrm{E}-04 \\
(1.302 \mathrm{E}-05)\end{array}$ & $\begin{array}{c}1.905 \mathrm{E}-04 \\
(1.075 \mathrm{E}-05)\end{array}$ & $\begin{array}{l}\ldots \\
(\ldots)\end{array}$ & $\begin{array}{c}1.019 \mathrm{E}-03 \\
(8.443 \mathrm{E}-05)\end{array}$ & $\begin{array}{c}1.770 \mathrm{E}-03 \\
(1.630 \mathrm{E}-04)\end{array}$ & $\begin{array}{l}\ldots \\
(\ldots)\end{array}$ & $\begin{array}{c}3.404 \mathrm{E}-03 \\
(2.195 \mathrm{E}-04)\end{array}$ & $\begin{array}{c}7.850 \mathrm{E}-03 \\
(4.966 \mathrm{E}-05)\end{array}$ & $\begin{array}{c}1.001 \mathrm{E}-02 \\
(5.567 \mathrm{E}-05)\end{array}$ & $\begin{array}{l}\ldots \\
(\ldots)\end{array}$ \\
\hline UGC 02982 & $\begin{array}{l}\ldots \\
(\ldots)\end{array}$ & $\begin{array}{l}\ldots \\
(\ldots)\end{array}$ & $\begin{array}{c}3.468 \mathrm{E}-05 \\
(3.076 \mathrm{E}-05)\end{array}$ & $\begin{array}{c}4.028 \mathrm{E}-05 \\
(1.637 \mathrm{E}-05)\end{array}$ & $\begin{array}{l}\ldots \\
(\ldots)\end{array}$ & $\begin{array}{c}1.690 \mathrm{E}-03 \\
(1.090 \mathrm{E}-04)\end{array}$ & $\begin{array}{c}4.699 \mathrm{E}-03 \\
(1.731 \mathrm{E}-04)\end{array}$ & $\begin{array}{c}9.651 \mathrm{E}-03 \\
(6.222 \mathrm{E}-04)\end{array}$ & $\begin{array}{c}1.259 \mathrm{E}-02 \\
(4.638 \mathrm{E}-04)\end{array}$ & $\begin{array}{c}5.635 \mathrm{E}-02 \\
(1.349 \mathrm{E}-03)\end{array}$ & $\begin{array}{c}7.398 \mathrm{E}-02 \\
(1.976 \mathrm{E}-03)\end{array}$ & $\begin{array}{c}7.920 \mathrm{E}-02 \\
(2.261 \mathrm{E}-03)\end{array}$ \\
\hline ESO 550-IG025 & $\begin{array}{l}\ldots \\
(\ldots)\end{array}$ & $\begin{array}{l}\ldots \\
(\ldots)\end{array}$ & $\begin{array}{c}1.992 \mathrm{E}-04 \\
(2.459 \mathrm{E}-05)\end{array}$ & $\begin{array}{c}4.222 \mathrm{E}-04 \\
(2.384 \mathrm{E}-05)\end{array}$ & $\begin{array}{l}\ldots \\
(\ldots)\end{array}$ & $\begin{array}{c}3.873 \mathrm{E}-03 \\
(2.140 \mathrm{E}-04)\end{array}$ & $\begin{array}{c}7.178 \mathrm{E}-03 \\
(3.967 \mathrm{E}-04)\end{array}$ & $\begin{array}{c}7.187 \mathrm{E}-03 \\
(4.634 \mathrm{E}-04)\end{array}$ & $\begin{array}{c}1.445 \mathrm{E}-02 \\
(7.988 \mathrm{E}-04)\end{array}$ & $\begin{array}{c}3.564 \mathrm{E}-02 \\
(1.120 \mathrm{E}-04)\end{array}$ & $\begin{array}{c}4.766 \mathrm{E}-02 \\
(3.408 \mathrm{E}-04)\end{array}$ & $\begin{array}{c}3.978 \mathrm{E}-02 \\
(1.785 \mathrm{E}-04)\end{array}$ \\
\hline NGC 1614 & $\begin{array}{l}\ldots \\
(\ldots)\end{array}$ & $\begin{array}{l}\ldots \\
(\ldots)\end{array}$ & $\begin{array}{l}\ldots \\
(\ldots)\end{array}$ & $\begin{array}{l}\ldots \\
(\ldots)\end{array}$ & $\begin{array}{l}1.307 \mathrm{E}-02^{\mathrm{a}} \\
(1.686 \mathrm{E}-04)\end{array}$ & $\begin{array}{c}1.330 \mathrm{E}-02 \\
(9.803 \mathrm{E}-04)\end{array}$ & $\begin{array}{c}2.188 \mathrm{E}-02 \\
(1.209 \mathrm{E}-03)\end{array}$ & $\begin{array}{l}3.255 \mathrm{E}-02^{\mathrm{a}} \\
(5.996 \mathrm{E}-04)\end{array}$ & $\begin{array}{c}4.246 \mathrm{E}-02 \\
(2.347 \mathrm{E}-03)\end{array}$ & $\begin{array}{c}9.204 \mathrm{E}-02 \\
(1.678 \mathrm{E}-04)\end{array}$ & $\begin{array}{c}1.173 \mathrm{E}-01 \\
(2.079 \mathrm{E}-04)\end{array}$ & $\begin{array}{c}1.127 \mathrm{E}-01 \\
(2.372 \mathrm{E}-04)\end{array}$ \\
\hline IRAS F05189-2524 & $\begin{array}{c}2.143 \mathrm{E}-07 \\
(\ldots)\end{array}$ & $\begin{array}{c}2.691 \mathrm{E}-08 \\
(\ldots)\end{array}$ & $\begin{array}{c}8.816 \mathrm{E}-05 \\
(1.088 \mathrm{E}-05)\end{array}$ & $\begin{array}{c}2.079 \mathrm{E}-04 \\
(1.174 \mathrm{E}-05)\end{array}$ & $\begin{array}{l}1.905 \mathrm{E}-03^{\mathrm{b}} \\
(3.510 \mathrm{E}-04)\end{array}$ & $\begin{array}{l}2.291 \mathrm{E}-03^{\mathrm{b}} \\
(4.220 \mathrm{E}-04)\end{array}$ & $\begin{array}{c}2.589 \mathrm{E}-03 \\
(4.770 \mathrm{E}-04)\end{array}$ & $\begin{array}{c}5.065 \mathrm{E}-03 \\
(3.265 \mathrm{E}-04)\end{array}$ & $\begin{array}{c}7.805 \mathrm{E}-03 \\
(3.595 \mathrm{E}-03)\end{array}$ & $\begin{array}{c}1.698 \mathrm{E}-02 \\
(8.018 \mathrm{E}-05)\end{array}$ & $\begin{array}{c}2.655 \mathrm{E}-02 \\
(1.383 \mathrm{E}-04)\end{array}$ & $\begin{array}{c}4.770 \mathrm{E}-02 \\
(1.273 \mathrm{E}-04)\end{array}$ \\
\hline NGC 2623 & $\begin{array}{l}\ldots \\
(\ldots)\end{array}$ & $\begin{array}{l}\ldots \\
(\ldots)\end{array}$ & $\begin{array}{c}3.904 \mathrm{E}-04 \\
(4.820 \mathrm{E}-05)\end{array}$ & $\begin{array}{c}7.948 \mathrm{E}-04 \\
(4.487 \mathrm{E}-05)\end{array}$ & $\begin{array}{l}5.592 \mathrm{E}-03^{\mathrm{a}} \\
(1.082 \mathrm{E}-04)\end{array}$ & $\begin{array}{c}7.656 \mathrm{E}-03 \\
(2.115 \mathrm{E}-04)\end{array}$ & $\begin{array}{c}1.282 \mathrm{E}-02 \\
(3.543 \mathrm{E}-04)\end{array}$ & $\begin{array}{l}1.797 \mathrm{E}-02^{\mathrm{a}} \\
(3.476 \mathrm{E}-04)\end{array}$ & $\begin{array}{c}2.188 \mathrm{E}-02 \\
(4.030 \mathrm{E}-04)\end{array}$ & $\begin{array}{c}3.795 \mathrm{E}-02 \\
(1.127 \mathrm{E}-04)\end{array}$ & $\begin{array}{c}5.127 \mathrm{E}-02 \\
(2.758 \mathrm{E}-04)\end{array}$ & $\begin{array}{c}4.667 \mathrm{E}-02 \\
(1.670 \mathrm{E}-04)\end{array}$ \\
\hline IRAS F08572+3915 & $\begin{array}{c}\text { 1.654E-09 } \\
(\ldots)\end{array}$ & $\begin{array}{c}3.177 \mathrm{E}-09 \\
(\ldots)\end{array}$ & $\begin{array}{c}8.223 \mathrm{E}-05 \\
(1.015 \mathrm{E}-05)\end{array}$ & $\begin{array}{c}1.269 \mathrm{E}-04 \\
(7.164 \mathrm{E}-06)\end{array}$ & $\begin{array}{l}6.918 \mathrm{E}-04^{\mathrm{b}} \\
(1.274 \mathrm{E}-04)\end{array}$ & $\begin{array}{l}6.710 \mathrm{E}-04^{\mathrm{b}} \\
(1.233 \mathrm{E}-05)\end{array}$ & $\begin{array}{c}8.158 \mathrm{E}-04 \\
(2.254 \mathrm{E}-04)\end{array}$ & $\begin{array}{c}9.651 \mathrm{E}-04 \\
(6.222 \mathrm{E}-05)\end{array}$ & $\begin{array}{l}1.710 \mathrm{E}-03^{\mathrm{a}} \\
(1.353 \mathrm{E}-05)\end{array}$ & $\begin{array}{c}3.113 \mathrm{E}-03 \\
(3.883 \mathrm{E}-05)\end{array}$ & $\begin{array}{c}3.342 \mathrm{E}-03 \\
(5.757 \mathrm{E}-05)\end{array}$ & $\begin{array}{c}4.581 \mathrm{E}-03 \\
(5.571 \mathrm{E}-05)\end{array}$ \\
\hline UGC 04881 & $\begin{array}{c}9.824 \mathrm{E}-10 \\
(\ldots)\end{array}$ & $\begin{array}{c}4.542 \mathrm{E}-09 \\
(\ldots)\end{array}$ & $\begin{array}{c}2.150 \mathrm{E}-04 \\
(2.654 \mathrm{E}-05)\end{array}$ & $\begin{array}{c}4.792 \mathrm{E}-04 \\
(2.705 \mathrm{E}-05)\end{array}$ & $\begin{array}{l}\ldots \\
(\ldots)\end{array}$ & $\begin{array}{c}4.325 \mathrm{E}-03 \\
(2.390 \mathrm{E}-04)\end{array}$ & $\begin{array}{c}7.586 \mathrm{E}-03 \\
(4.891 \mathrm{E}-04)\end{array}$ & $\begin{array}{l}\ldots \\
(\ldots)\end{array}$ & $\begin{array}{c}1.486 \mathrm{E}-02 \\
(8.212 \mathrm{E}-04)\end{array}$ & $\begin{array}{c}3.011 \mathrm{E}-02 \\
(1.041 \mathrm{E}-04)\end{array}$ & $\begin{array}{c}3.465 \mathrm{E}-02 \\
(1.203 \mathrm{E}-04)\end{array}$ & $\begin{array}{c}3.689 \mathrm{E}-02 \\
(1.406 \mathrm{E}-04)\end{array}$ \\
\hline UGC 05101 & $\begin{array}{c}8.647 \mathrm{E}-09 \\
(\ldots)\end{array}$ & $\begin{array}{c}6.183 \mathrm{E}-09 \\
(\ldots)\end{array}$ & $\begin{array}{c}5.977 \mathrm{E}-05 \\
(7.378 \mathrm{E}-06)\end{array}$ & $\begin{array}{c}1.800 \mathrm{E}-04 \\
(1.016 \mathrm{E}-05)\end{array}$ & $\begin{array}{l}1.586 \mathrm{E}-03^{\mathrm{a}} \\
(5.990 \mathrm{E}-05)\end{array}$ & $\begin{array}{c}2.874 \mathrm{E}-03^{\mathrm{a}} \\
(4.9097 \mathrm{E}-05)\end{array}$ & $\begin{array}{l}5.124 \mathrm{E}-03^{\mathrm{a}} \\
(1.463 \mathrm{E}-04)\end{array}$ & $\begin{array}{c}1.434 \mathrm{E}-02 \\
(9.245 \mathrm{E}-04)\end{array}$ & $\begin{array}{l}1.0547 \mathrm{E}-02^{\mathrm{a}} \\
(9.7144 \mathrm{E}-05)\end{array}$ & $\begin{array}{c}2.233 \mathrm{E}-02 \\
(9.444 \mathrm{E}-05)\end{array}$ & $\begin{array}{c}3.087 \mathrm{E}-02 \\
(1.312 \mathrm{E}-04)\end{array}$ & $\begin{array}{c}3.960 \mathrm{E}-02 \\
(1.357 \mathrm{E}-04)\end{array}$ \\
\hline MCG +08-18-013 & $\begin{array}{l}\ldots \\
(\ldots)\end{array}$ & $(\ldots)$ & $\begin{array}{c}7.505 \mathrm{E}-04 \\
(3.076 \mathrm{E}-05)\end{array}$ & $\begin{array}{c}1.063 \mathrm{E}-03 \\
(1.637 \mathrm{E}-05)\end{array}$ & $(\ldots)$ & $\begin{array}{c}5.808 \mathrm{E}-03 \\
(5.349 \mathrm{E}-04)\end{array}$ & $\begin{array}{c}8.954 \mathrm{E}-03 \\
(6.597 \mathrm{E}-04)\end{array}$ & $\begin{array}{l}\ldots \\
(\ldots)\end{array}$ & $\begin{array}{c}1.675 \mathrm{E}-02 \\
(1.388 \mathrm{E}-03)\end{array}$ & $\begin{array}{c}2.152 \mathrm{E}-03 \\
(2.696 \mathrm{E}-04)\end{array}$ & $\begin{array}{c}3.133 \mathrm{E}-03 \\
(4.414 \mathrm{E}-04)\end{array}$ & $\begin{array}{c}2.288 \mathrm{E}-03 \\
(4.342 \mathrm{E}-04)\end{array}$ \\
\hline IC $0563 / 4$ & $\begin{array}{l}\ldots \\
(\ldots)\end{array}$ & $\begin{array}{l}\ldots \\
(\ldots)\end{array}$ & $\begin{array}{c}3.575 \mathrm{E}-04 \\
(2.307 \mathrm{E}-05)\end{array}$ & $\begin{array}{c}6.942 \mathrm{E}-04 \\
(1.637 \mathrm{E}-05)\end{array}$ & $\begin{array}{l}\ldots \\
(\ldots)\end{array}$ & $\begin{array}{c}1.247 \mathrm{E}-02 \\
(8.042 \mathrm{E}-04)\end{array}$ & $\begin{array}{c}2.148 \mathrm{E}-02 \\
(1.187 \mathrm{E}-03)\end{array}$ & $\begin{array}{l}3.1685 \mathrm{E}-02 \\
(5.837 \mathrm{E}-04)\end{array}$ & $\begin{array}{c}4.571 \mathrm{E}-02 \\
(2.526 \mathrm{E}-03)\end{array}$ & $\begin{array}{c}8.131 \mathrm{E}-02 \\
(3.162 \mathrm{E}-04)\end{array}$ & $\begin{array}{c}1.042 \mathrm{E}-01 \\
(4.702 \mathrm{E}-04)\end{array}$ & $\begin{array}{c}9.629 \mathrm{E}-02 \\
(3.714 \mathrm{E}-04)\end{array}$ \\
\hline
\end{tabular}


Table 4

Continued)

\begin{tabular}{|c|c|c|c|c|c|c|c|c|c|c|c|c|}
\hline RBGS Name & $\begin{array}{c}\mathrm{HX} \\
2-10 \mathrm{KeV} \\
(2) \\
\end{array}$ & $\begin{array}{c}\mathrm{SX} \\
0.5-2 \mathrm{KeV} \\
(3) \\
\end{array}$ & $\begin{array}{c}\text { FUV } \\
152.8 \AA \\
(4) \\
\end{array}$ & $\begin{array}{c}\text { NUV } \\
227.1 \AA \\
(5) \\
\end{array}$ & $\begin{array}{c}U \\
3655 \AA \\
(6) \\
\end{array}$ & $\begin{array}{c}B \\
4582 \AA \\
(7) \\
\end{array}$ & $\begin{array}{c}V \\
5377 \AA \\
(8)\end{array}$ & $\begin{array}{c}R \\
6484 \AA \\
(9) \\
\end{array}$ & $\begin{array}{c}I \\
8570 \AA \\
(10) \\
\end{array}$ & $\begin{array}{c}J \\
1.241 \mu \mathrm{m} \\
(11)\end{array}$ & $\begin{array}{c}H \\
1.651 \mu \mathrm{m} \\
(12)\end{array}$ & $\begin{array}{c}K_{\mathrm{s}} \\
2.165 \mu \mathrm{m} \\
(13)\end{array}$ \\
\hline NGC 3110 & $(\ldots)$ & $(\ldots)$ & $\begin{array}{c}1.384 \mathrm{E}-03 \\
(4.613 \mathrm{E}-05)\end{array}$ & $\begin{array}{c}2.734 \mathrm{E}-03 \\
(3.275 \mathrm{E}-05)\end{array}$ & $(\ldots)$ & $\begin{array}{c}1.794 \mathrm{E}-02 \\
(3.605 \mathrm{E}-03)\end{array}$ & $\begin{array}{c}2.731 \mathrm{E}-02 \\
(3.018 \mathrm{E}-03)\end{array}$ & $\begin{array}{l}1.517 \mathrm{E}-02^{\mathrm{a}} \\
(1.397 \mathrm{E}-03)\end{array}$ & $\begin{array}{c}5.077 \mathrm{E}-02 \\
(5.423 \mathrm{E}-03)\end{array}$ & $\begin{array}{c}9.130 \mathrm{E}-02 \\
(1.850 \mathrm{E}-03)\end{array}$ & $\begin{array}{c}1.142 \mathrm{E}-01 \\
(2.418 \mathrm{E}-03)\end{array}$ & $\begin{array}{c}1.031 \mathrm{E}-01 \\
(2.943 \mathrm{E}-03)\end{array}$ \\
\hline IRAS F10173+0828 & $\begin{array}{c}7.582 \mathrm{E}-11 \\
(\ldots)\end{array}$ & $\begin{array}{c}6.067 \mathrm{E}-10 \\
(\ldots)\end{array}$ & $\begin{array}{c}1.678 \mathrm{E}-06 \\
(2.072 \mathrm{E}-07)\end{array}$ & $\begin{array}{c}8.517 \mathrm{E}-06 \\
(4.809 \mathrm{E}-07)\end{array}$ & $\begin{array}{l}\ldots \\
(\ldots)\end{array}$ & $\begin{array}{c}4.406 \mathrm{E}-04 \\
(2.840 \mathrm{E}-05)\end{array}$ & $\begin{array}{c}1.009 \mathrm{E}-03 \\
(5.577 \mathrm{E}-05)\end{array}$ & $\begin{array}{c}2.758 \mathrm{E}-03 \\
(1.778 \mathrm{E}-04)\end{array}$ & $\begin{array}{c}2.249 \mathrm{E}-03 \\
(1.036 \mathrm{E}-04)\end{array}$ & $\begin{array}{c}3.817 \mathrm{E}-03 \\
(4.416 \mathrm{E}-05)\end{array}$ & $\begin{array}{c}4.703 \mathrm{E}-03 \\
(5.791 \mathrm{E}-05)\end{array}$ & $\begin{array}{c}3.315 \mathrm{E}-03 \\
(6.045 \mathrm{E}-05)\end{array}$ \\
\hline IRAS F10565+2448 & $\begin{array}{c}2.376 \mathrm{E}-09 \\
(\ldots)\end{array}$ & $\begin{array}{c}8.557 \mathrm{E}-09 \\
(\ldots)\end{array}$ & $\begin{array}{l}\ldots \\
(\ldots)\end{array}$ & $\begin{array}{l}\ldots \\
(\ldots)\end{array}$ & $\begin{array}{c}2.160 \mathrm{E}-03 \\
(5.968 \mathrm{E}-04)\end{array}$ & $\begin{array}{c}3.862 \mathrm{E}-03^{\mathrm{a}} \\
(1.067 \mathrm{E}-03)\end{array}$ & $\begin{array}{c}5.541 \mathrm{E}-03 \\
(1.531 \mathrm{E}-03)\end{array}$ & $\begin{array}{c}8.483 \mathrm{E}-03 \\
(5.469 \mathrm{E}-04)\end{array}$ & $\begin{array}{l}1.319 \mathrm{E}-02^{\mathrm{a}} \\
(3.646 \mathrm{E}-03)\end{array}$ & $\begin{array}{c}1.949 \mathrm{E}-02 \\
(9.129 \mathrm{E}-05)\end{array}$ & $\begin{array}{c}2.313 \mathrm{E}-02 \\
(1.236 \mathrm{E}-04)\end{array}$ & $\begin{array}{c}2.584 \mathrm{E}-02 \\
(1.211 \mathrm{E}-04)\end{array}$ \\
\hline MCG +07-23-019 & $\begin{array}{l}\ldots \\
(\ldots)\end{array}$ & $\begin{array}{l}\ldots \\
(\ldots)\end{array}$ & $\begin{array}{c}4.708 \mathrm{E}-04 \\
(5.812 \mathrm{E}-05)\end{array}$ & $\begin{array}{c}7.601 \mathrm{E}-04 \\
(4.291 \mathrm{E}-05)\end{array}$ & $\begin{array}{l}\ldots \\
(\ldots)\end{array}$ & $\begin{array}{c}5.861 \mathrm{E}-03 \\
(3.239 \mathrm{E}-04)\end{array}$ & $\begin{array}{c}8.472 \mathrm{E}-03 \\
(4.682 \mathrm{E}-04)\end{array}$ & $\begin{array}{c}8.801 \mathrm{E}-03 \\
(5.674 \mathrm{E}-04)\end{array}$ & $\begin{array}{c}1.542 \mathrm{E}-02 \\
(8.520 \mathrm{E}-04)\end{array}$ & $\begin{array}{c}1.508 \mathrm{E}-02 \\
(6.866 \mathrm{E}-05)\end{array}$ & $\begin{array}{c}1.931 \mathrm{E}-02 \\
(1.031 \mathrm{E}-04)\end{array}$ & $\begin{array}{c}1.971 \mathrm{E}-02 \\
(8.964 \mathrm{E}-05)\end{array}$ \\
\hline CGCG 011-076 & $\begin{array}{l}\ldots \\
(\ldots)\end{array}$ & $\begin{array}{l}\ldots \\
(\ldots)\end{array}$ & $\begin{array}{c}4.537 \mathrm{E}-05 \\
(7.689 \mathrm{E}-06)\end{array}$ & $\begin{array}{c}1.541 \mathrm{E}-04 \\
(9.824 \mathrm{E}-06)\end{array}$ & $\begin{array}{l}\ldots \\
(\ldots)\end{array}$ & $\begin{array}{c}3.311 \mathrm{E}-03 \\
(1.220 \mathrm{E}-04)\end{array}$ & $\begin{array}{c}7.112 \mathrm{E}-03 \\
(1.965 \mathrm{E}-04)\end{array}$ & $\begin{array}{l}9.921 \mathrm{E}-03 \\
(6.396 \mathrm{E}-04)\end{array}$ & $\begin{array}{c}1.871 \mathrm{E}-02 \\
(5.169 \mathrm{E}-04)\end{array}$ & $\begin{array}{c}3.645 \mathrm{E}-02 \\
(6.378 \mathrm{E}-04)\end{array}$ & $\begin{array}{c}4.807 \mathrm{E}-02 \\
(9.741 \mathrm{E}-04)\end{array}$ & $\begin{array}{c}4.524 \mathrm{E}-02 \\
(1.208 \mathrm{E}-03)\end{array}$ \\
\hline IC 2810 & $\begin{array}{l}\ldots \\
(\ldots)\end{array}$ & $\begin{array}{l}\ldots \\
(\ldots)\end{array}$ & $\begin{array}{c}1.164 \mathrm{E}-04 \\
(1.437 \mathrm{E}-05)\end{array}$ & $\begin{array}{c}3.110 \mathrm{E}-04 \\
(1.756 \mathrm{E}-05)\end{array}$ & $\begin{array}{l}\ldots \\
(\ldots)\end{array}$ & $\begin{array}{c}4.325 \mathrm{E}-03 \\
(5.179 \mathrm{E}-04)\end{array}$ & $\begin{array}{c}9.036 \mathrm{E}-03 \\
(1.082 \mathrm{E}-03)\end{array}$ & $\begin{array}{c}2.151 \mathrm{E}-02 \\
(1.387 \mathrm{E}-03)\end{array}$ & $\begin{array}{c}1.837 \mathrm{E}-02 \\
(2.030 \mathrm{E}-03)\end{array}$ & $\begin{array}{c}3.454 \mathrm{E}-02 \\
(8.969 \mathrm{E}-05)\end{array}$ & $\begin{array}{c}4.451 \mathrm{E}-02 \\
(1.505 \mathrm{E}-04)\end{array}$ & $\begin{array}{c}3.866 \mathrm{E}-02 \\
(1.220 \mathrm{E}-04)\end{array}$ \\
\hline NGC 3690/IC 694 & $\begin{array}{c}5.830 \mathrm{E}-08 \\
(\ldots)\end{array}$ & $\begin{array}{c}2.047 \mathrm{E}-07 \\
(\ldots)\end{array}$ & $\begin{array}{c}8.798 \mathrm{E}-03 \\
(1.086 \mathrm{E}-03)\end{array}$ & $\begin{array}{c}1.373 \mathrm{E}-02 \\
(7.750 \mathrm{E}-04)\end{array}$ & (..) & $\begin{array}{c}4.966 \mathrm{E}-02 \\
(9.148 \mathrm{E}-04)\end{array}$ & $\begin{array}{c}7.244 \mathrm{E}-02 \\
(1.334 \mathrm{E}-03)\end{array}$ & $\begin{array}{c}7.056 \mathrm{E}-02 \\
(4.549 \mathrm{E}-03)\end{array}$ & $\begin{array}{c}1.294 \mathrm{E}-01 \\
(2.384 \mathrm{E}-03)\end{array}$ & $\begin{array}{c}2.170 \mathrm{E}-01 \\
(2.525 \mathrm{E}-04)\end{array}$ & $\begin{array}{c}3.064 \mathrm{E}-01 \\
(3.995 \mathrm{E}-04)\end{array}$ & $\begin{array}{c}2.810 \mathrm{E}-01 \\
(3.149 \mathrm{E}-04)\end{array}$ \\
\hline IRAS F12112+0305 & $\begin{array}{c}1.994 \mathrm{E}-09 \\
(\ldots)\end{array}$ & $\begin{array}{c}3.112 \mathrm{E}-09 \\
(\ldots)\end{array}$ & $\begin{array}{l}\ldots \\
(\ldots)\end{array}$ & $\begin{array}{l}\ldots \\
(\ldots)\end{array}$ & $\begin{array}{l}8.318 \mathrm{E}-04^{\mathrm{b}} \\
(1.532 \mathrm{E}-04)\end{array}$ & $\begin{array}{l}8.250 \mathrm{E}-04^{\mathrm{b}} \\
(1.520 \mathrm{E}-05)\end{array}$ & $\begin{array}{c}1.133 \mathrm{E}-03 \\
(3.132 \mathrm{E}-04)\end{array}$ & $\begin{array}{c}2.211 \mathrm{E}-03 \\
(1.425 \mathrm{E}-04)\end{array}$ & $\begin{array}{l}2.398 \mathrm{E}-03^{\mathrm{b}} \\
(2.398 \mathrm{E}-05)\end{array}$ & $\begin{array}{c}4.835 \mathrm{E}-03 \\
(5.406 \mathrm{E}-05)\end{array}$ & $\begin{array}{l}5.941 \mathrm{E}-03 \\
(6.844 \mathrm{E}-05)\end{array}$ & $\begin{array}{c}5.278 \mathrm{E}-03 \\
(8.116 \mathrm{E}-05)\end{array}$ \\
\hline IRAS F12224-0624 & $\begin{array}{l}\ldots \\
(\ldots)\end{array}$ & $\begin{array}{l}\ldots \\
(\ldots)\end{array}$ & $\begin{array}{l}\ldots \\
(\ldots)\end{array}$ & $\begin{array}{l}\ldots \\
(\ldots)\end{array}$ & $\begin{array}{l}\ldots \\
(\ldots)\end{array}$ & $\begin{array}{c}8.166 \mathrm{E}-04 \\
(4.513 \mathrm{E}-05)\end{array}$ & $\begin{array}{c}1.660 \mathrm{E}-03 \\
(7.643 \mathrm{E}-05)\end{array}$ & $\begin{array}{c}3.024 \mathrm{E}-03 \\
(1.950 \mathrm{E}-04)\end{array}$ & $\begin{array}{c}3.162 \mathrm{E}-03 \\
(1.165 \mathrm{E}-04)\end{array}$ & $\begin{array}{c}5.045 \mathrm{E}-03 \\
(4.507 \mathrm{E}-04)\end{array}$ & $\begin{array}{c}6.431 \mathrm{E}-03 \\
(4.620 \mathrm{E}-04)\end{array}$ & $\begin{array}{c}5.474 \mathrm{E}-03 \\
(5.950 \mathrm{E}-04)\end{array}$ \\
\hline UGC 08058 & $\begin{array}{c}4.691 \mathrm{E}-08 \\
(\ldots)\end{array}$ & $\begin{array}{c}2.928 \mathrm{E}-08 \\
(\ldots)\end{array}$ & $\begin{array}{l}\ldots \\
(\ldots)\end{array}$ & $\begin{array}{l}\ldots \\
(\ldots)\end{array}$ & $\begin{array}{l}7.586 \mathrm{E}-03^{b} \\
(1.397 \mathrm{E}-03)\end{array}$ & $\begin{array}{l}1.202 \mathrm{E}-02^{\mathrm{b}} \\
(2.215 \mathrm{E}-03)\end{array}$ & $\begin{array}{l}1.282 \mathrm{E}-02^{\mathrm{a}} \\
(2.008 \mathrm{E}-03)\end{array}$ & $\begin{array}{c}1.616 \mathrm{E}-02 \\
(1.042 \mathrm{E}-03)\end{array}$ & $\begin{array}{c}2.585 \mathrm{E}-02 \\
(1.190 \mathrm{E}-02)\end{array}$ & $\begin{array}{c}6.412 \mathrm{E}-02 \\
(1.214 \mathrm{E}-04)\end{array}$ & $\begin{array}{c}1.147 \mathrm{E}-01 \\
(1.747 \mathrm{E}-04)\end{array}$ & $\begin{array}{c}1.991 \mathrm{E}-01 \\
(1.944 \mathrm{E}-04)\end{array}$ \\
\hline NGC 4922 & $\begin{array}{l}\ldots \\
(\ldots)\end{array}$ & $\begin{array}{l}\ldots \\
(\ldots)\end{array}$ & $\begin{array}{c}1.076 \mathrm{E}-04 \\
(3.845 \mathrm{E}-06)\end{array}$ & $\begin{array}{c}3.586 \mathrm{E}-04 \\
(3.275 \mathrm{E}-06)\end{array}$ & $\begin{array}{l}\ldots \\
(\ldots)\end{array}$ & $\begin{array}{c}1.213 \mathrm{E}-02 \\
(4.470 \mathrm{E}-04)\end{array}$ & $\begin{array}{c}2.377 \mathrm{E}-02 \\
(8.757 \mathrm{E}-04)\end{array}$ & $\begin{array}{l}\ldots \\
(\ldots)\end{array}$ & $\begin{array}{c}1.038 \mathrm{E}-01 \\
(2.867 \mathrm{E}-03)\end{array}$ & $\begin{array}{c}7.266 \mathrm{E}-02 \\
(1.205 \mathrm{E}-03)\end{array}$ & $\begin{array}{c}9.356 \mathrm{E}-02 \\
(1.982 \mathrm{E}-03)\end{array}$ & $\begin{array}{c}7.761 \mathrm{E}-02 \\
(1.859 \mathrm{E}-03)\end{array}$ \\
\hline ESO 507-G070 & $\begin{array}{l}\ldots \\
(\ldots)\end{array}$ & $\begin{array}{l}\ldots \\
(\ldots)\end{array}$ & $\begin{array}{c}1.360 \mathrm{E}-04 \\
(1.679 \mathrm{E}-05)\end{array}$ & $\begin{array}{c}2.331 \mathrm{E}-04 \\
(1.316 \mathrm{E}-05)\end{array}$ & $\begin{array}{l}\ldots \\
(\ldots)\end{array}$ & $\begin{array}{c}4.571 \mathrm{E}-03 \\
(3.368 \mathrm{E}-04)\end{array}$ & $\begin{array}{c}9.204 \mathrm{E}-03 \\
(5.087 \mathrm{E}-04)\end{array}$ & $\begin{array}{l}1.558 \mathrm{E}-02^{\mathrm{a}} \\
(1.291 \mathrm{E}-03)\end{array}$ & $\begin{array}{c}2.148 \mathrm{E}-02 \\
(1.187 \mathrm{E}-03)\end{array}$ & $\begin{array}{c}4.512 \mathrm{E}-02 \\
(1.472 \mathrm{E}-04)\end{array}$ & $\begin{array}{c}6.025 \mathrm{E}-02 \\
(2.671 \mathrm{E}-04)\end{array}$ & $\begin{array}{c}5.426 \mathrm{E}-02 \\
(2.081 \mathrm{E}-04)\end{array}$ \\
\hline IC 0860 & $\begin{array}{l}\ldots \\
(\ldots)\end{array}$ & $\begin{array}{l}\ldots \\
(\ldots)\end{array}$ & $\begin{array}{l}\ldots \\
(\ldots)\end{array}$ & $\begin{array}{l}\ldots \\
(\ldots)\end{array}$ & $\begin{array}{l}\ldots \\
(\ldots)\end{array}$ & $\begin{array}{c}6.368 \mathrm{E}-03 \\
(1.760 \mathrm{E}-04)\end{array}$ & $\begin{array}{c}1.180 \mathrm{E}-02 \\
(3.261 \mathrm{E}-04)\end{array}$ & $\begin{array}{c}1.740 \mathrm{E}-02 \\
(1.122 \mathrm{E}-03)\end{array}$ & $\begin{array}{c}2.228 \mathrm{E}-02 \\
(4.105 \mathrm{E}-04)\end{array}$ & $\begin{array}{c}3.778 \mathrm{E}-02 \\
(6.264 \mathrm{E}-04)\end{array}$ & $\begin{array}{c}4.856 \mathrm{E}-02 \\
(8.946 \mathrm{E}-04)\end{array}$ & $\begin{array}{c}3.865 \mathrm{E}-02 \\
(9.967 \mathrm{E}-04)\end{array}$ \\
\hline VV 250a & $\begin{array}{c}8.933 \mathrm{E}-09 \\
(\ldots)\end{array}$ & $\begin{array}{c}1.825 \mathrm{E}-08 \\
(\ldots)\end{array}$ & $\begin{array}{c}6.253 \mathrm{E}-04 \\
(7.719 \mathrm{E}-05)\end{array}$ & $\begin{array}{c}1.051 \mathrm{E}-03 \\
(5.931 \mathrm{E}-05)\end{array}$ & $\begin{array}{l}\ldots \\
(\ldots)\end{array}$ & $\begin{array}{c}6.310 \mathrm{E}-03 \\
(5.811 \mathrm{E}-04)\end{array}$ & $\begin{array}{c}8.954 \mathrm{E}-03 \\
(6.597 \mathrm{E}-04)\end{array}$ & $\begin{array}{l}\ldots \\
(\ldots)\end{array}$ & $\begin{array}{c}1.486 \mathrm{E}-02 \\
(8.212 \mathrm{E}-04)\end{array}$ & $\begin{array}{c}2.408 \mathrm{E}-02 \\
(8.872 \mathrm{E}-05)\end{array}$ & $\begin{array}{c}3.223 \mathrm{E}-02 \\
(1.484 \mathrm{E}-04)\end{array}$ & $\begin{array}{l}\ldots \\
(\ldots)\end{array}$ \\
\hline UGC 08387 & $\begin{array}{c}3.049 \mathrm{E}-09 \\
(\ldots)\end{array}$ & $\begin{array}{c}1.235-08 \\
(\ldots)\end{array}$ & $\begin{array}{c}3.117 \mathrm{E}-04 \\
(3.847 \mathrm{E}-05)\end{array}$ & $\begin{array}{c}6.781 \mathrm{E}-04 \\
(3.829 \mathrm{E}-05)\end{array}$ & $\begin{array}{l}\ldots \\
(\ldots)\end{array}$ & $\begin{array}{c}6.792 \mathrm{E}-03 \\
(3.128 \mathrm{E}-04)\end{array}$ & $\begin{array}{c}9.908 \mathrm{E}-03 \\
(2.738 \mathrm{E}-04)\end{array}$ & $\begin{array}{c}1.068 \mathrm{E}-02 \\
(6.885 \mathrm{E}-04)\end{array}$ & $\begin{array}{c}1.941 \mathrm{E}-02 \\
(5.363 \mathrm{E}-04)\end{array}$ & $\begin{array}{c}3.084 \mathrm{E}-02 \\
(1.035 \mathrm{E}-04)\end{array}$ & $\begin{array}{c}3.846 \mathrm{E}-02 \\
(1.231 \mathrm{E}-04)\end{array}$ & $\begin{array}{c}3.581 \mathrm{E}-02 \\
(1.431 \mathrm{E}-04)\end{array}$ \\
\hline NGC 5104 & $\begin{array}{l}\ldots \\
(\ldots)\end{array}$ & $\begin{array}{l}\ldots \\
(\ldots)\end{array}$ & $\begin{array}{c}2.284 \mathrm{E}-04 \\
(1.538 \mathrm{E}-05)\end{array}$ & $\begin{array}{c}4.732 \mathrm{E}-04 \\
(1.637 \mathrm{E}-05)\end{array}$ & $\begin{array}{l}\ldots \\
(\ldots)\end{array}$ & $\begin{array}{c}7.727 \mathrm{E}-03 \\
(2.135 \mathrm{E}-04)\end{array}$ & $\begin{array}{c}1.500 \mathrm{E}-02 \\
(4.144 \mathrm{E}-04)\end{array}$ & $\begin{array}{l}\ldots \\
(\ldots)\end{array}$ & $\begin{array}{c}3.631 \mathrm{E}-02 \\
(1.003 \mathrm{E}-03)\end{array}$ & $\begin{array}{c}7.153 \mathrm{E}-02 \\
(1.976 \mathrm{E}-03)\end{array}$ & $\begin{array}{c}8.935 \mathrm{E}-02 \\
(2.963 \mathrm{E}-03)\end{array}$ & $\begin{array}{c}8.440 \mathrm{E}-02 \\
(2.177 \mathrm{E}-03)\end{array}$ \\
\hline MCG -03-34-064 & $\begin{array}{c}1.448 \mathrm{E}-07^{\mathrm{a}} \\
(\ldots)\end{array}$ & $\begin{array}{c}1.192 \mathrm{E}-07^{\mathrm{a}} \\
(\ldots)\end{array}$ & $\begin{array}{c}2.337 \mathrm{E}-04 \\
(2.307 \mathrm{E}-05)\end{array}$ & $\begin{array}{c}4.011 \mathrm{E}-04 \\
(1.637 \mathrm{E}-05)\end{array}$ & (... & $\begin{array}{c}1.330 \mathrm{E}-02 \\
(1.470 \mathrm{E}-03)\end{array}$ & $\begin{array}{c}2.704 \mathrm{E}-02 \\
(2.989 \mathrm{E}-03)\end{array}$ & $\begin{array}{l}\ldots \\
(\ldots)\end{array}$ & $\begin{array}{c}5.702 \mathrm{E}-02 \\
(6.302 \mathrm{E}-03)\end{array}$ & $\begin{array}{c}7.650 \mathrm{E}-02 \\
(1.409 \mathrm{E}-03)\end{array}$ & $\begin{array}{c}9.689 \mathrm{E}-02 \\
(1.785 \mathrm{E}-03)\end{array}$ & $\begin{array}{c}8.401 \mathrm{E}-02 \\
(2.089 \mathrm{E}-03)\end{array}$ \\
\hline NGC 5135 & $\begin{array}{c}3.840 \mathrm{E}-08^{\mathrm{a}} \\
(\ldots)\end{array}$ & $\begin{array}{c}1.775 \mathrm{E}-07^{\mathrm{a}} \\
(\ldots)\end{array}$ & $\begin{array}{c}1.384 \mathrm{E}-03 \\
(4.613 \mathrm{E}-05)\end{array}$ & $\begin{array}{c}3.209 \mathrm{E}-03 \\
(3.275 \mathrm{E}-05)\end{array}$ & $\begin{array}{l}2.070 \mathrm{E}-02^{\mathrm{a}} \\
(2.669 \mathrm{E}-03)\end{array}$ & $\begin{array}{c}2.858 \mathrm{E}-02 \\
(7.896 \mathrm{E}-04)\end{array}$ & $\begin{array}{c}5.445 \mathrm{E}-02 \\
(1.505 \mathrm{E}-03)\end{array}$ & $\begin{array}{l}\ldots \\
(\ldots)\end{array}$ & $\begin{array}{c}1.202 \mathrm{E}-01 \\
(2.215 \mathrm{E}-03)\end{array}$ & $\begin{array}{c}1.797 \mathrm{E}-01 \\
(3.641 \mathrm{E}-03)\end{array}$ & $\begin{array}{c}2.615 \mathrm{E}-01 \\
(5.058 \mathrm{E}-03)\end{array}$ & $\begin{array}{c}1.959 \mathrm{E}-01 \\
(5.772 \mathrm{E}-03)\end{array}$ \\
\hline NGC 5256 & $\begin{array}{c}2.483 \mathrm{E}-08^{\mathrm{a}} \\
(\ldots)\end{array}$ & $\begin{array}{c}9.934 \mathrm{E}-09^{\mathrm{a}} \\
(\ldots)\end{array}$ & $\begin{array}{c}8.090 \mathrm{E}-04 \\
(9.987 \mathrm{E}-05)\end{array}$ & $\begin{array}{c}1.419 \mathrm{E}-03 \\
(8.014 \mathrm{E}-05)\end{array}$ & $\begin{array}{l}\ldots \\
(\ldots)\end{array}$ & $\begin{array}{c}1.038 \mathrm{E}-02 \\
(1.911 \mathrm{E}-04)\end{array}$ & $\begin{array}{c}1.871 \mathrm{E}-02 \\
(3.446 \mathrm{E}-04)\end{array}$ & $\begin{array}{l}2.538 \mathrm{E}-02^{\mathrm{a}} \\
(4.676 \mathrm{E}-04)\end{array}$ & $\begin{array}{c}3.311 \mathrm{E}-02 \\
(6.100 \mathrm{E}-04)\end{array}$ & $\begin{array}{c}5.266 \mathrm{E}-02 \\
(1.486 \mathrm{E}-04)\end{array}$ & $\begin{array}{c}6.512 \mathrm{E}-02 \\
(2.356 \mathrm{E}-04)\end{array}$ & $\begin{array}{c}6.266 \mathrm{E}-02 \\
(2.117 \mathrm{E}-04)\end{array}$ \\
\hline NGC 5257/8 & $\begin{array}{l}\ldots \\
(\ldots)\end{array}$ & $\begin{array}{l}\ldots \\
(\ldots)\end{array}$ & $\begin{array}{c}3.319 \mathrm{E}-03 \\
(4.097 \mathrm{E}-04)\end{array}$ & $\begin{array}{c}5.743 \mathrm{E}-03 \\
(3.243 \mathrm{E}-04)\end{array}$ & $\begin{array}{l}\ldots \\
(\ldots)\end{array}$ & $\begin{array}{c}2.679 \mathrm{E}-02 \\
(2.961 \mathrm{E}-03)\end{array}$ & $\begin{array}{c}4.325 \mathrm{E}-02 \\
(4.780 \mathrm{E}-03)\end{array}$ & $\begin{array}{c}5.978 \mathrm{E}-02 \\
(3.854 \mathrm{E}-03)\end{array}$ & $\begin{array}{c}\text { 7.447E-02 } \\
(8.231 \mathrm{E}-03)\end{array}$ & $\begin{array}{c}1.314 \mathrm{E}-01 \\
(2.502 \mathrm{E}-04)\end{array}$ & $\begin{array}{c}1.680 \mathrm{E}-01 \\
(3.197 \mathrm{E}-04)\end{array}$ & $\begin{array}{c}1.477 \mathrm{E}-01 \\
(3.580 \mathrm{E}-04)\end{array}$ \\
\hline UGC 08696 & $\begin{array}{c}4.815 \mathrm{E}-08 \\
(\ldots)\end{array}$ & $\begin{array}{c}2.866 \mathrm{E}-07 \\
(\ldots)\end{array}$ & $\begin{array}{c}2.039 \mathrm{E}-04 \\
(2.517 \mathrm{E}-05)\end{array}$ & $\begin{array}{c}4.057 \mathrm{E}-04 \\
(2.291 \mathrm{E}-05)\end{array}$ & $\begin{array}{l}3.020 \mathrm{E}-03^{\mathrm{b}} \\
(5.563 \mathrm{E}-04)\end{array}$ & $\begin{array}{l}5.297 \mathrm{E}-03^{\mathrm{b}} \\
(3.415 \mathrm{E}-04)\end{array}$ & $\begin{array}{c}9.036 \mathrm{E}-03 \\
(2.497 \mathrm{E}-03)\end{array}$ & $\begin{array}{c}9.301 \mathrm{E}-03 \\
(5.997 \mathrm{E}-04)\end{array}$ & $\begin{array}{l}1.380 \mathrm{E}-02^{\mathrm{b}} \\
(8.900 \mathrm{E}-04)\end{array}$ & $\begin{array}{c}3.042 \mathrm{E}-02 \\
(1.055 \mathrm{E}-04)\end{array}$ & $\begin{array}{c}3.385 \mathrm{E}-02 \\
(1.462 \mathrm{E}-04)\end{array}$ & $\begin{array}{c}3.581 \mathrm{E}-02 \\
(1.410 \mathrm{E}-04)\end{array}$ \\
\hline CGCG 247-020 & $\begin{array}{l}\ldots \\
(\ldots)\end{array}$ & $\begin{array}{l}\ldots \\
(\ldots)\end{array}$ & $\begin{array}{c}3.591 \mathrm{E}-05 \\
(1.538 \mathrm{E}-05)\end{array}$ & $\begin{array}{c}5.878 \mathrm{E}-05 \\
(1.146 \mathrm{E}-05)\end{array}$ & $\begin{array}{l}\ldots \\
(\ldots)\end{array}$ & $\begin{array}{c}1.941 \mathrm{E}-03 \\
(7.150 \mathrm{E}-05)\end{array}$ & $\begin{array}{c}3.664 \mathrm{E}-03 \\
(4.388 \mathrm{E}-04)\end{array}$ & $\begin{array}{l}\ldots \\
(\ldots)\end{array}$ & $\begin{array}{c}7.727 \mathrm{E}-03 \\
(7.828 \mathrm{E}-04)\end{array}$ & $\begin{array}{c}1.518 \mathrm{E}-02 \\
(4.754 \mathrm{E}-04)\end{array}$ & $\begin{array}{c}2.097 \mathrm{E}-02 \\
(7.145 \mathrm{E}-04)\end{array}$ & $\begin{array}{c}2.010 \mathrm{E}-02 \\
(8.144 \mathrm{E}-04)\end{array}$ \\
\hline IRAS F14348-1447 & $\begin{array}{c}2.886 \mathrm{E}-09 \\
(\ldots)\end{array}$ & $\begin{array}{c}5.359 \mathrm{E}-09 \\
(\ldots)\end{array}$ & $\begin{array}{c}6.417 \mathrm{E}-05 \\
(7.921 \mathrm{E}-06)\end{array}$ & $\begin{array}{c}1.288 \mathrm{E}-04 \\
(7.274 \mathrm{E}-06)\end{array}$ & $\begin{array}{l}5.754 \mathrm{E}-04^{\mathrm{b}} \\
(1.060 \mathrm{E}-04)\end{array}$ & $\begin{array}{l}7.922 \mathrm{E}-04^{\mathrm{b}} \\
(1.678 \mathrm{E}-05)\end{array}$ & $\begin{array}{c}1.265 \mathrm{E}-03 \\
(3.495 \mathrm{E}-04)\end{array}$ & $\begin{array}{c}2.424 \mathrm{E}-03 \\
(1.563 \mathrm{E}-04)\end{array}$ & $\begin{array}{l}3.217 \mathrm{E}-03^{\mathrm{b}} \\
(5.926 \mathrm{E}-05)\end{array}$ & $\begin{array}{c}5.621 \mathrm{E}-03 \\
(5.092 \mathrm{E}-05)\end{array}$ & $\begin{array}{c}7.847 \mathrm{E}-03 \\
(7.111 \mathrm{E}-05)\end{array}$ & $\begin{array}{c}9.000 \mathrm{E}-03 \\
(8.989 \mathrm{E}-05)\end{array}$ \\
\hline
\end{tabular}




\begin{tabular}{|c|c|c|c|c|c|c|c|c|c|c|c|c|}
\hline RBGS Name & $\begin{array}{c}\mathrm{HX} \\
2-10 \mathrm{KeV} \\
(2)\end{array}$ & $\begin{array}{c}\text { SX } \\
0.5-2 \mathrm{KeV} \\
\text { (3) }\end{array}$ & $\begin{array}{c}\text { FUV } \\
152.8 \AA \\
(4)\end{array}$ & $\begin{array}{l}\text { NUV } \\
227.1 \AA \\
(5)\end{array}$ & $\begin{array}{c}U \\
3655 \AA \\
(6)\end{array}$ & $\begin{array}{c}B \\
4582 \AA \\
(7)\end{array}$ & $\begin{array}{c}V \\
5377 \AA \\
(8)\end{array}$ & $\begin{array}{c}R \\
6484 \AA \\
(9)\end{array}$ & $\begin{array}{c}I \\
8570 \AA ̊ \\
(10)\end{array}$ & $\begin{array}{c}J \\
1.241 \mu \mathrm{m} \\
(11)\end{array}$ & $\begin{array}{c}H \\
1.651 \mu \mathrm{m} \\
(12)\end{array}$ & $\begin{array}{c}K_{\mathrm{S}} \\
2.165 \mu \mathrm{m} \\
(13)\end{array}$ \\
\hline VV 340a & $\begin{array}{c}7.015 \mathrm{E}-09 \\
(\ldots)\end{array}$ & $\begin{array}{c}1.684 \mathrm{E}-08 \\
(\ldots)\end{array}$ & $\begin{array}{c}4.185 \mathrm{E}-04 \\
(5.166 \mathrm{E}-05)\end{array}$ & $\begin{array}{c}7.972 \mathrm{E}-04 \\
(4.501 \mathrm{E}-05)\end{array}$ & $(\ldots)$ & $\begin{array}{c}7.112 \mathrm{E}-03 \\
(7.861 \mathrm{E}-04)\end{array}$ & $\begin{array}{c}1.406 \mathrm{E}-02 \\
(1.554 \mathrm{E}-03)\end{array}$ & $\begin{array}{c}1.369 \mathrm{E}-02 \\
(8.829 \mathrm{E}-04)\end{array}$ & $\begin{array}{c}2.679 \mathrm{E}-02 \\
(2.961 \mathrm{E}-03)\end{array}$ & $\begin{array}{c}6.066 \mathrm{E}-02 \\
(1.117 \mathrm{E}-04)\end{array}$ & $\begin{array}{c}9.391 \mathrm{E}-02 \\
(2.595 \mathrm{E}-04)\end{array}$ & $\begin{array}{c}7.571 \mathrm{E}-02 \\
(2.092 \mathrm{E}-04)\end{array}$ \\
\hline CGCG 049-057 & $(\ldots)$ & $(\ldots)$ & $\begin{array}{c}6.051 \mathrm{E}-05 \\
(1.538 \mathrm{E}-06)\end{array}$ & $\begin{array}{c}1.524 \mathrm{E}-04 \\
(1.474 \mathrm{E}-05)\end{array}$ & $\begin{array}{l}\ldots \\
(\ldots)\end{array}$ & $\begin{array}{c}2.228 \mathrm{E}-03 \\
(8.210 \mathrm{E}-05)\end{array}$ & $\begin{array}{c}4.786 \mathrm{E}-03 \\
(1.763 \mathrm{E}-04)\end{array}$ & $\begin{array}{l}\ldots \\
(\ldots)\end{array}$ & $\begin{array}{c}1.086 \mathrm{E}-02 \\
(3.002 \mathrm{E}-04)\end{array}$ & $\begin{array}{c}2.140 \mathrm{E}-02 \\
(6.703 \mathrm{E}-04)\end{array}$ & $\begin{array}{c}2.875 \mathrm{E}-02 \\
(8.210 \mathrm{E}-04)\end{array}$ & $\begin{array}{c}2.366 \mathrm{E}-02 \\
(1.002 \mathrm{E}-03)\end{array}$ \\
\hline VV 705 & $\begin{array}{c}1.308 \mathrm{E}-09 \\
(\ldots)\end{array}$ & $\begin{array}{c}9.917 \mathrm{E}-09 \\
(\ldots)\end{array}$ & $\begin{array}{c}3.411 \mathrm{E}-04 \\
(4.210 \mathrm{E}-05)\end{array}$ & $\begin{array}{c}6.693 \mathrm{E}-04 \\
(3.779 \mathrm{E}-05)\end{array}$ & $\begin{array}{l}\ldots \\
(\ldots)\end{array}$ & $\begin{array}{c}4.325 \mathrm{E}-03 \\
(1.992 \mathrm{E}-04)\end{array}$ & $\begin{array}{c}6.368 \mathrm{E}-03 \\
(1.760 \mathrm{E}-04)\end{array}$ & $\begin{array}{c}9.651 \mathrm{E}-03 \\
(6.222 \mathrm{E}-04)\end{array}$ & $\begin{array}{c}1.247 \mathrm{E}-02 \\
(3.447 \mathrm{E}-04)\end{array}$ & $\begin{array}{c}1.907 \mathrm{E}-02 \\
(8.558 \mathrm{E}-05)\end{array}$ & $\begin{array}{c}2.473 \mathrm{E}-02 \\
(1.259 \mathrm{E}-04)\end{array}$ & $\begin{array}{c}2.244 \mathrm{E}-02 \\
(1.190 \mathrm{E}-04)\end{array}$ \\
\hline IRAS F15250+3608 & $\begin{array}{c}4.020 \mathrm{E}-10 \\
(\ldots)\end{array}$ & $\begin{array}{c}2.660 \mathrm{E}-09 \\
(\ldots)\end{array}$ & $\begin{array}{l}\ldots \\
(\ldots)\end{array}$ & $\begin{array}{l}\ldots \\
(\ldots)\end{array}$ & $\begin{array}{l}1.096 \mathrm{E}-03^{b} \\
(2.020 \mathrm{E}-04)\end{array}$ & $\begin{array}{c}1.247 \mathrm{E}-03 \\
(5.744 \mathrm{E}-05)\end{array}$ & $\begin{array}{c}1.905 \mathrm{E}-03 \\
(8.775 \mathrm{E}-05)\end{array}$ & $\begin{array}{c}2.707 \mathrm{E}-03 \\
(1.746 \mathrm{E}-04)\end{array}$ & $\begin{array}{c}2.606 \mathrm{E}-03 \\
(3.529 \mathrm{E}-04)\end{array}$ & $\begin{array}{c}5.308 \mathrm{E}-03 \\
(4.821 \mathrm{E}-05)\end{array}$ & $\begin{array}{c}7.152 \mathrm{E}-03 \\
(7.816 \mathrm{E}-05)\end{array}$ & $\begin{array}{c}6.044 \mathrm{E}-03 \\
(7.617 \mathrm{E}-05)\end{array}$ \\
\hline UGC 09913 & $\begin{array}{c}5.073 \mathrm{E}-09 \\
(\ldots)\end{array}$ & $\begin{array}{c}7.164 \mathrm{E}-09 \\
(\ldots)\end{array}$ & $\begin{array}{c}1.313 \mathrm{E}-04 \\
(1.620 \mathrm{E}-05)\end{array}$ & $\begin{array}{c}4.371 \mathrm{E}-04 \\
(2.468 \mathrm{E}-05)\end{array}$ & $\begin{array}{l}6.076 \mathrm{E}-03^{\mathrm{a}} \\
(1.287 \mathrm{E}-04)\end{array}$ & $\begin{array}{l}9.272 \mathrm{E}-03^{\mathrm{b}} \\
(1.708 \mathrm{E}-04)\end{array}$ & $\begin{array}{l}1.893 \mathrm{E}-02^{\mathrm{a}} \\
(3.261 \mathrm{E}-04)\end{array}$ & $\begin{array}{c}2.131 \mathrm{E}-02 \\
(1.374 \mathrm{E}-03)\end{array}$ & $\begin{array}{l}3.619 \mathrm{E}-02^{\mathrm{b}} \\
(3.333 \mathrm{E}-04)\end{array}$ & $\begin{array}{c}7.260 \mathrm{E}-02 \\
(1.669 \mathrm{E}-04)\end{array}$ & $\begin{array}{c}8.793 \mathrm{E}-02 \\
(2.400 \mathrm{E}-04)\end{array}$ & $\begin{array}{c}9.187 \mathrm{E}-02 \\
(2.459 \mathrm{E}-04)\end{array}$ \\
\hline NGC & $\begin{array}{l}\ldots \\
(\ldots)\end{array}$ & $\begin{array}{l}\ldots \\
(\ldots)\end{array}$ & $\begin{array}{c}1.094 \mathrm{E}-03 \\
(1.350 \mathrm{E}-04)\end{array}$ & $\begin{array}{c}1.915 \mathrm{E}-03 \\
(1.081 \mathrm{E}-04)\end{array}$ & $\begin{array}{l}\ldots \\
(\ldots)\end{array}$ & $\begin{array}{c}7.379 \mathrm{E}-03 \\
(6.796 \mathrm{E}-04)\end{array}$ & $\begin{array}{c}1.057 \mathrm{E}-02 \\
(5.840 \mathrm{E}-04)\end{array}$ & $\begin{array}{c}1.789 \mathrm{E}-02 \\
(1.153 \mathrm{E}-03)\end{array}$ & $\begin{array}{c}1.820 \mathrm{E}-02 \\
(5.028 \mathrm{E}-04)\end{array}$ & $\begin{array}{c}2.933 \mathrm{E}-02 \\
(1.180 \mathrm{E}-04)\end{array}$ & $\begin{array}{c}3.433 \mathrm{E}-02 \\
(1.637 \mathrm{E}-04)\end{array}$ & $\begin{array}{c}3.492 \mathrm{E}-02 \\
(1.673 \mathrm{E}-04)\end{array}$ \\
\hline CGCG & $\begin{array}{l}\ldots \\
(\ldots)\end{array}$ & $\begin{array}{l}\ldots \\
(\ldots)\end{array}$ & $\begin{array}{c}1.192 \mathrm{E}-04 \\
(1.538 \mathrm{E}-05)\end{array}$ & $\begin{array}{c}3.029 \mathrm{E}-04 \\
(1.474 \mathrm{E}-05)\end{array}$ & $\begin{array}{l}\ldots \\
(\ldots)\end{array}$ & & $\begin{array}{c}8.790 \mathrm{E}-03 \\
(3.238 \mathrm{E}-04)\end{array}$ & $\begin{array}{c}1.344 \mathrm{E}-02 \\
(8.668 \mathrm{E}-04)\end{array}$ & $\begin{array}{c}1.854 \mathrm{E}-02 \\
(5.121 \mathrm{E}-04)\end{array}$ & $\begin{array}{c}3.655 \mathrm{E}-02 \\
(7.069 \mathrm{E}-04)\end{array}$ & $\begin{array}{c}4.583 \mathrm{E}-02 \\
(1.266 \mathrm{E}-04)\end{array}$ & $\begin{array}{c}4.516 \mathrm{E}-02 \\
(1.206 \mathrm{E}-03)\end{array}$ \\
\hline NGC & $(\ldots)$ & $(\ldots)$ & $\begin{array}{c}5.628 \mathrm{E}-04 \\
(2.307 \mathrm{E}-05)\end{array}$ & $\begin{array}{c}1.058 \mathrm{E}-03 \\
(1.637 \mathrm{E}-05)\end{array}$ & $\begin{array}{l}\ldots \\
(\ldots)\end{array}$ & $\begin{array}{c}1.542 \mathrm{E}-02 \\
(1.988 \mathrm{E}-03)\end{array}$ & $\begin{array}{c}2.443 \mathrm{E}-02 \\
(3.151 \mathrm{E}-03)\end{array}$ & $\begin{array}{c}6.260 \mathrm{E}-02 \\
(4.036 \mathrm{E}-03)\end{array}$ & $\begin{array}{c}5.058 \mathrm{E}-02 \\
(6.056 \mathrm{E}-03)\end{array}$ & $\begin{array}{c}5.687 \mathrm{E}-02 \\
(8.381 \mathrm{E}-04)\end{array}$ & $\begin{array}{c}8.224 \mathrm{E}-02 \\
(1.363 \mathrm{E}-03)\end{array}$ & $\begin{array}{c}8.301 \mathrm{E}-02 \\
(3.364 \mathrm{E}-03)\end{array}$ \\
\hline IRAS & $\begin{array}{c}9.210 \mathrm{E}-10 \\
(\ldots)\end{array}$ & $\begin{array}{c}4.268 \mathrm{E}-09 \\
(\ldots)\end{array}$ & $\begin{array}{c}3.538 \mathrm{E}-05 \\
(4.367 \mathrm{E}-06)\end{array}$ & $\begin{array}{c}9.769 \mathrm{E}-05 \\
(5.515 \mathrm{E}-06)\end{array}$ & $\begin{array}{l}\ldots \\
(\ldots)\end{array}$ & $\begin{array}{c}7.311 \mathrm{E}-04 \\
(3.367 \mathrm{E}-05)\end{array}$ & $\begin{array}{c}1.486 \mathrm{E}-03 \\
(5.474 \mathrm{E}-05)\end{array}$ & $\begin{array}{c}2.941 \mathrm{E}-03 \\
(1.896 \mathrm{E}-04)\end{array}$ & $\begin{array}{c}2.679 \mathrm{E}-03 \\
(9.870 \mathrm{E}-05)\end{array}$ & $\begin{array}{r}1.168 \mathrm{I} \\
(5.672 \mathrm{I}\end{array}$ & $\begin{array}{c}1.593 \mathrm{E}-02 \\
(7.121 \mathrm{E}-05)\end{array}$ & $\begin{array}{c}1.576 \mathrm{E}-02 \\
(7.730 \mathrm{E}-05)\end{array}$ \\
\hline ESO & $\begin{array}{l}\ldots \\
(\ldots)\end{array}$ & $\begin{array}{l}\ldots \\
(\ldots)\end{array}$ & $\begin{array}{c}2.791 \mathrm{E}-04 \\
(3.076 \mathrm{E}-05)\end{array}$ & $\begin{array}{c}5.534 \mathrm{E}-04 \\
(3.275 \mathrm{E}-05)\end{array}$ & $\begin{array}{l}\ldots \\
(\ldots)\end{array}$ & $\begin{array}{c}6.668 \mathrm{E}-03 \\
(1.842 \mathrm{E}-04)\end{array}$ & $\begin{array}{c}1.159 \mathrm{E}-02 \\
(3.202 \mathrm{E}-04)\end{array}$ & $\begin{array}{c}1.772 \mathrm{E}-02 \\
(1.143 \mathrm{E}-03)\end{array}$ & $\begin{array}{c}2.400 \mathrm{E}-02 \\
(1.547 \mathrm{E}-03)\end{array}$ & $\begin{array}{c}4.930 \mathrm{E}-02 \\
(1.135 \mathrm{E}-03)\end{array}$ & $\begin{array}{c}6.419 \mathrm{E}-02 \\
(1.774 \mathrm{E}-03)\end{array}$ & $\begin{array}{c}5.785 \mathrm{E}-02 \\
(1.865 \mathrm{E}-03)\end{array}$ \\
\hline IRAS F22491-1808 & $\begin{array}{c}2.807 \mathrm{E}-10 \\
(\ldots)\end{array}$ & $\begin{array}{c}2.920 \mathrm{E}-09 \\
(\ldots)\end{array}$ & $\begin{array}{c}1.673 \mathrm{E}-04 \\
(2.066 \mathrm{E}-05)\end{array}$ & $\begin{array}{c}2.348 \mathrm{E}-04 \\
(1.326 \mathrm{E}-05)\end{array}$ & $\begin{array}{l}4.365 \mathrm{E}-04^{\mathrm{b}} \\
(8.041 \mathrm{E}-05)\end{array}$ & $\begin{array}{l}9.543 \mathrm{E}-04^{\mathrm{b}} \\
(1.758 \mathrm{E}-05)\end{array}$ & $\begin{array}{c}8.551 \mathrm{E}-04 \\
(1.575 \mathrm{E}-04)\end{array}$ & $\begin{array}{c}2.131 \mathrm{E}-03 \\
(1.374 \mathrm{E}-04)\end{array}$ & $\begin{array}{l}2.399 \mathrm{E}-03^{\mathrm{b}} \\
(1.547 \mathrm{E}-04)\end{array}$ & $\begin{array}{c}3.972 \mathrm{E}-03 \\
(3.651 \mathrm{E}-05)\end{array}$ & $\begin{array}{c}4.923 \mathrm{E}-03 \\
(5.112 \mathrm{E}-05)\end{array}$ & $\begin{array}{c}4.808 \mathrm{E}-03 \\
(5.686 \mathrm{E}-05)\end{array}$ \\
\hline NGC 7 & $\begin{array}{c}1.906 \mathrm{E}-06^{\mathrm{a}} \\
(\ldots)\end{array}$ & $\begin{array}{l}\ldots \\
(\ldots)\end{array}$ & $\begin{array}{c}4.322 \mathrm{E}-03 \\
(5.335 \mathrm{E}-04)\end{array}$ & $\begin{array}{c}5.948 \mathrm{E}-03 \\
(3.358 \mathrm{E}-04)\end{array}$ & $\begin{array}{l}3.020 \mathrm{E}-02^{\mathrm{b}} \\
(5.563 \mathrm{E}-03)\end{array}$ & $\begin{array}{c}2.890 \mathrm{E}-02 \\
(1.863 \mathrm{E}-03)\end{array}$ & $\begin{array}{c}4.713 \mathrm{E}-02 \\
(2.605 \mathrm{E}-03)\end{array}$ & $\begin{array}{c}6.202 \mathrm{E}-02 \\
(3.999 \mathrm{E}-03)\end{array}$ & $\begin{array}{c}9.290 \mathrm{E}-02 \\
(3.422 \mathrm{E}-03)\end{array}$ & $\begin{array}{c}1.915 \mathrm{E}-01 \\
(2.486 \mathrm{E}-04)\end{array}$ & $\begin{array}{c}2.369 \mathrm{E}-01 \\
(3.390 \mathrm{E}-04)\end{array}$ & $\begin{array}{c}2.356 \mathrm{E}-01 \\
(3.269 \mathrm{E}-04)\end{array}$ \\
\hline CGCG & $\begin{array}{l}\ldots \\
(\ldots)\end{array}$ & $\begin{array}{l}\ldots \\
(\ldots)\end{array}$ & $\begin{array}{c}5.774 \mathrm{E}-05 \\
(1.538 \mathrm{E}-05)\end{array}$ & $\begin{array}{c}2.112 \mathrm{E}-04 \\
(1.474 \mathrm{E}-05)\end{array}$ & $\begin{array}{l}\ldots \\
(\ldots)\end{array}$ & $\begin{array}{c}3.436 \mathrm{E}-03 \\
(1.266 \mathrm{E}-04)\end{array}$ & $\begin{array}{c}6.427 \mathrm{E}-03 \\
(1.776 \mathrm{E}-04)\end{array}$ & $\begin{array}{l}\ldots \\
(\ldots)\end{array}$ & $\begin{array}{c}1.330 \mathrm{E}-02 \\
(3.676 \mathrm{E}-04)\end{array}$ & $\begin{array}{c}3.219 \mathrm{E}-02 \\
(6.522 \mathrm{E}-04)\end{array}$ & $\begin{array}{c}4.111 \mathrm{E}-02 \\
(8.708 \mathrm{E}-04)\end{array}$ & $\begin{array}{c}3.861 \mathrm{E}-02 \\
(1.174 \mathrm{E}-03)\end{array}$ \\
\hline IC 5298 & $\begin{array}{l}\ldots \\
(\ldots)\end{array}$ & $\begin{array}{l}\ldots \\
(\ldots)\end{array}$ & $\begin{array}{c}1.220 \mathrm{E}-04 \\
(1.506 \mathrm{E}-05)\end{array}$ & $\begin{array}{c}2.934 \mathrm{E}-04 \\
(1.656 \mathrm{E}-05)\end{array}$ & $\begin{array}{l}\ldots \\
(\ldots)\end{array}$ & $\begin{array}{c}3.597 \mathrm{E}-03 \\
(1.325 \mathrm{E}-04)\end{array}$ & $\begin{array}{c}8.166 \mathrm{E}-03 \\
(2.256 \mathrm{E}-04)\end{array}$ & $\begin{array}{c}1.182 \mathrm{E}-02 \\
(7.619 \mathrm{E}-04)\end{array}$ & $\begin{array}{c}1.854 \mathrm{E}-02 \\
(6.829 \mathrm{E}-04)\end{array}$ & $\begin{array}{c}3.481 \mathrm{E}-02 \\
(1.130 \mathrm{E}-04)\end{array}$ & $\begin{array}{c}4.963 \mathrm{E}-02 \\
(1.563 \mathrm{E}-04)\end{array}$ & $\begin{array}{c}4.860 \mathrm{E}-02 \\
(1.678 \mathrm{E}-04)\end{array}$ \\
\hline NGC 7592 & $\begin{array}{l}\ldots \\
(\ldots)\end{array}$ & $\begin{array}{l}\ldots \\
(\ldots)\end{array}$ & $\begin{array}{c}1.784 \mathrm{E}-03 \\
(4.613 \mathrm{E}-05)\end{array}$ & $\begin{array}{c}2.259 \mathrm{E}-03 \\
(3.275 \mathrm{E}-05)\end{array}$ & $\begin{array}{l}\ldots \\
(\ldots)\end{array}$ & $\begin{array}{c}9.638 \mathrm{E}-03 \\
(3.551 \mathrm{E}-04)\end{array}$ & $\begin{array}{c}1.393 \mathrm{E}-02 \\
(3.849 \mathrm{E}-04)\end{array}$ & $\begin{array}{c}1.926 \mathrm{E}-02 \\
(1.241 \mathrm{E}-03)\end{array}$ & $\begin{array}{c}2.606 \mathrm{E}-02 \\
(7.201 \mathrm{E}-04)\end{array}$ & $\begin{array}{c}4.509 \mathrm{E}-02 \\
(6.645 \mathrm{E}-04)\end{array}$ & $\begin{array}{c}5.325 \mathrm{E}-02 \\
(1.275 \mathrm{E}-03)\end{array}$ & $\begin{array}{c}3.351 \mathrm{E}-02 \\
(1.142 \mathrm{E}-03)\end{array}$ \\
\hline NGC 7674 & $\begin{array}{c}4.828 \mathrm{E}-08^{\mathrm{a}} \\
(\ldots)\end{array}$ & $\begin{array}{c}7.430 \mathrm{E}-08^{\mathrm{a}} \\
(\ldots)\end{array}$ & $\begin{array}{c}1.325 \mathrm{E}-03 \\
(1.636 \mathrm{E}-04)\end{array}$ & $\begin{array}{c}1.937 \mathrm{E}-03 \\
(1.094 \mathrm{E}-04)\end{array}$ & $\begin{array}{l}9.638 \mathrm{E}-03^{\mathrm{a}} \\
(9.765 \mathrm{E}-04)\end{array}$ & $\begin{array}{c}1.228 \mathrm{E}-02 \\
(6.783 \mathrm{E}-04)\end{array}$ & $\begin{array}{c}2.109 \mathrm{E}-02 \\
(5.826 \mathrm{E}-04)\end{array}$ & $\begin{array}{c}3.196 \mathrm{E}-02 \\
(2.060 \mathrm{E}-03)\end{array}$ & $\begin{array}{c}3.908 \mathrm{E}-02 \\
(7.200 \mathrm{E}-04)\end{array}$ & $\begin{array}{c}7.315 \mathrm{E}-02 \\
(1.672 \mathrm{E}-04)\end{array}$ & $\begin{array}{c}1.114 \mathrm{E}-01 \\
(2.620 \mathrm{E}-04)\end{array}$ & $\begin{array}{c}8.746 \mathrm{E}-02 \\
(2.150 \mathrm{E}-04)\end{array}$ \\
\hline NGC 7770/1 & $\begin{array}{l}\ldots \\
(\ldots)\end{array}$ & $\begin{array}{l}\ldots \\
(\ldots)\end{array}$ & $\begin{array}{c}2.607 \mathrm{E}-03 \\
(6.151 \mathrm{E}-06)\end{array}$ & $\begin{array}{c}3.848 \mathrm{E}-03 \\
(3.275 \mathrm{E}-06)\end{array}$ & $\begin{array}{l}1.419 \mathrm{E}-02^{\mathrm{a}} \\
(1.307 \mathrm{E}-03)\end{array}$ & $\begin{array}{c}3.076 \mathrm{E}-02 \\
(8.500 \mathrm{E}-04)\end{array}$ & $\begin{array}{c}6.194 \mathrm{E}-02 \\
(1.141 \mathrm{E}-03)\end{array}$ & $\begin{array}{c}9.562 \mathrm{E}-02 \\
(6.165 \mathrm{E}-03)\end{array}$ & $\begin{array}{c}1.486 \mathrm{E}-01 \\
(2.737 \mathrm{E}-03)\end{array}$ & $\begin{array}{c}2.609 \mathrm{E}-01 \\
(2.643 \mathrm{E}-03)\end{array}$ & $\begin{array}{c}3.335 \mathrm{E}-01 \\
(4.300 \mathrm{E}-03)\end{array}$ & $\begin{array}{c}3.053 \mathrm{E}-01 \\
(4.780 \mathrm{E}-03)\end{array}$ \\
\hline MRK 0331 & (... & $\begin{array}{c}4.000 \mathrm{E}-08^{\mathrm{a}} \\
(\ldots)\end{array}$ & $\begin{array}{c}6.128 \mathrm{E}-05 \\
(4.215 \mathrm{E}-06)\end{array}$ & $\begin{array}{c}2.691 \mathrm{E}-04 \\
(8.629 \mathrm{E}-06)\end{array}$ & $\begin{array}{l}\ldots \\
(\ldots)\end{array}$ & $\begin{array}{c}5.740 \mathrm{E}-03 \\
(4.170 \mathrm{E}-04)\end{array}$ & $\begin{array}{c}1.110 \mathrm{E}-02 \\
(8.060 \mathrm{E}-04)\end{array}$ & $\begin{array}{c}1.732 \mathrm{E}-02 \\
(1.117 \mathrm{E}-03)\end{array}$ & $\begin{array}{c}2.584 \mathrm{E}-02 \\
(7.650 \mathrm{E}-04)\end{array}$ & $\begin{array}{c}6.500 \mathrm{E}-02 \\
(1.197 \mathrm{E}-04)\end{array}$ & $\begin{array}{c}8.684 \mathrm{E}-02 \\
(1.600 \mathrm{E}-04)\end{array}$ & $\begin{array}{c}8.355 \mathrm{E}-02 \\
(1.539 \mathrm{E}-04)\end{array}$ \\
\hline
\end{tabular}

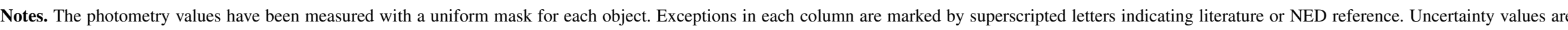

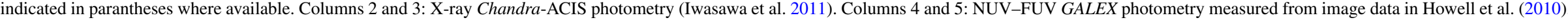

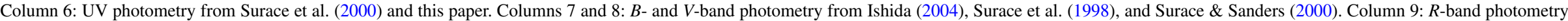
from J. M. Mazzarella et al. (2012, in preparation). Column 10: $I$-band photometry from Ishida (2004), Surace et al. (1998), and Surace \& Sanders (2000). Columns $11-13: J$, $H, K_{\mathrm{S}}$ photometry from 2MASS.

${ }^{a} \mathrm{NED}$

${ }^{b}$ Surace et al. $(1998,2000)$ and Surace \& Sanders (2000) 
Table 5

Local (U)LIRG Photometry (Jy; $\lambda>3 \mu \mathrm{m}$ )

\begin{tabular}{|c|c|c|c|c|c|c|c|c|c|c|c|c|c|c|c|}
\hline Object & $\begin{array}{c}\text { IRAC1 } \\
3.6 \mu \mathrm{m} \\
(2)\end{array}$ & $\begin{array}{c}\text { IRAC2 } \\
4.5 \mu \mathrm{m} \\
(3)\end{array}$ & $\begin{array}{l}\text { IRAC3 } \\
5.8 \mu \mathrm{m} \\
(4)\end{array}$ & $\begin{array}{c}\text { IRAC4 } \\
8.0 \mu \mathrm{m} \\
(5)\end{array}$ & $\begin{array}{c}\text { IRAS1 } \\
12 \mu \mathrm{m} \\
(6)\end{array}$ & $\begin{array}{l}\text { MIPS1 } \\
24 \mu \mathrm{m} \\
(7)\end{array}$ & $\begin{array}{l}\text { IRAS2 } \\
25 \mu \mathrm{m} \\
(8)\end{array}$ & $\begin{array}{c}\text { IRAS3 } \\
60 \mu \mathrm{m} \\
(9)\end{array}$ & $\begin{array}{c}\text { MIPS2 } \\
70 \mu \mathrm{m} \\
(10)\end{array}$ & $\begin{array}{c}\text { IRAS4 } \\
100 \mu \mathrm{m} \\
(11)\end{array}$ & $\begin{array}{c}\text { MIPS3 } \\
160 \mu \mathrm{m} \\
(12)\end{array}$ & $\begin{array}{c}\text { SCUBA1 } \\
450 \mu \mathrm{m} \\
(13)\end{array}$ & $\begin{array}{c}\text { SCUBA2 } \\
850 \mu \mathrm{m} \\
(14)\end{array}$ & $\begin{array}{c}\text { VLA1 } \\
4.85 \mathrm{GHz} \\
(15)\end{array}$ & $\begin{array}{c}\text { VLA2 } \\
1.49 \mathrm{GHz} \\
(16)\end{array}$ \\
\hline NGC 0034 & $\begin{array}{c}0.050 \\
(0.005)\end{array}$ & $\begin{array}{c}0.053 \\
(0.005)\end{array}$ & $\begin{array}{c}0.119 \\
(0.005)\end{array}$ & $\begin{array}{c}0.314 \\
(0.005)\end{array}$ & $\begin{array}{c}0.350 \\
(0.032)\end{array}$ & $\begin{array}{c}1.999 \\
(0.100)\end{array}$ & $\begin{array}{c}2.390 \\
(0.055)\end{array}$ & $\begin{array}{l}17.050 \\
(0.045)\end{array}$ & $\begin{array}{l}13.285 \\
(1.993)\end{array}$ & $\begin{array}{l}16.860 \\
(0.135)\end{array}$ & $\begin{array}{l}12.867 \\
(3.860)\end{array}$ & $\begin{array}{l}\ldots \\
(\ldots)\end{array}$ & $(\ldots)$ & $\begin{array}{l}\ldots \\
(\ldots)\end{array}$ & $\begin{array}{c}0.061 \\
(0.001)\end{array}$ \\
\hline MCG -02-01-051/2 & $\begin{array}{c}0.040 \\
(0.004)\end{array}$ & $\begin{array}{c}0.029 \\
(0.003)\end{array}$ & $\begin{array}{c}0.081 \\
(0.004)\end{array}$ & $\begin{array}{c}0.225 \\
(0.004)\end{array}$ & $\begin{array}{c}0.280 \\
(0.034)\end{array}$ & $\begin{array}{c}1.120 \\
(0.056)\end{array}$ & $\begin{array}{c}1.200 \\
(0.055)\end{array}$ & $\begin{array}{c}7.480 \\
(0.048)\end{array}$ & $\begin{array}{c}7.472 \\
(1.121)\end{array}$ & $\begin{array}{c}9.660 \\
(0.138)\end{array}$ & $\begin{array}{c}9.120 \\
(2.736)\end{array}$ & $\begin{array}{l}\ldots \\
(\ldots)\end{array}$ & $\begin{array}{l}\ldots \\
(\ldots)\end{array}$ & $\begin{array}{l}\ldots \\
(\ldots)\end{array}$ & $\begin{array}{c}0.041 \\
(0.001)\end{array}$ \\
\hline NGC 0232 & $\begin{array}{c}0.098 \\
(0.010)\end{array}$ & $\begin{array}{l}0.071 \\
(0.007)\end{array}$ & $\begin{array}{c}0.129 \\
(0.010)\end{array}$ & $\begin{array}{c}0.276 \\
(0.010)\end{array}$ & $\begin{array}{c}0.360 \\
(0.034)\end{array}$ & $\begin{array}{c}1.223 \\
(0.061)\end{array}$ & $\begin{array}{l}1.280 \\
(0.039)\end{array}$ & $\begin{array}{l}10.050 \\
(0.037)\end{array}$ & $\begin{array}{l}12.341 \\
(1.851)\end{array}$ & $\begin{array}{l}17.140 \\
(0.094)\end{array}$ & $\begin{array}{l}16.965 \\
(5.090)\end{array}$ & $\begin{array}{l}\ldots \\
(\ldots)\end{array}$ & $\begin{array}{l}\ldots \\
(\ldots)\end{array}$ & $\begin{array}{l}\ldots \\
(\ldots)\end{array}$ & $\begin{array}{c}0.052 \\
(0.001)\end{array}$ \\
\hline IC 1623A/B & $\begin{array}{c}0.092 \\
(0.009)\end{array}$ & $\begin{array}{c}0.099 \\
(0.010)\end{array}$ & $\begin{array}{c}0.241 \\
(0.009)\end{array}$ & $\begin{array}{c}0.542 \\
(0.009)\end{array}$ & $\begin{array}{c}1.030 \\
(0.030)\end{array}$ & $\begin{array}{c}3.014 \\
(0.151)\end{array}$ & $\begin{array}{c}3.650 \\
(0.050)\end{array}$ & $\begin{array}{c}22.930 \\
(0.062)\end{array}$ & $\begin{array}{l}\ldots \\
(\ldots)\end{array}$ & $\begin{array}{c}31.550 \\
(0.113)\end{array}$ & $\begin{array}{l}\ldots \\
(\ldots)\end{array}$ & $\begin{array}{l}\ldots \\
(\ldots)\end{array}$ & $\begin{array}{l}\ldots \\
(\ldots)\end{array}$ & $\begin{array}{c}0.096 \\
(0.012)\end{array}$ & $\begin{array}{c}0.221 \\
(0.001)\end{array}$ \\
\hline MCG -03-04-014 & $\begin{array}{c}0.032 \\
(0.003)\end{array}$ & $\begin{array}{c}0.023 \\
(0.002)\end{array}$ & $\begin{array}{c}0.072 \\
(0.003)\end{array}$ & $\begin{array}{c}0.209 \\
(0.003)\end{array}$ & $\begin{array}{c}0.340 \\
(0.043)\end{array}$ & $\begin{array}{c}0.742 \\
(0.037)\end{array}$ & $\begin{array}{c}0.900 \\
(0.036)\end{array}$ & $\begin{array}{c}7.250 \\
(0.060)\end{array}$ & $\begin{array}{c}7.440 \\
(1.116)\end{array}$ & $\begin{array}{l}10.330 \\
(0.136)\end{array}$ & $\begin{array}{c}9.626 \\
(2.888)\end{array}$ & $\begin{array}{l}\ldots \\
(\ldots)\end{array}$ & $\begin{array}{l}\ldots \\
(\ldots)\end{array}$ & $\begin{array}{l}\ldots \\
(\ldots)\end{array}$ & $\begin{array}{c}0.040 \\
(0.001)\end{array}$ \\
\hline CGCG 436-030 & $\begin{array}{c}0.044 \\
(0.004)\end{array}$ & $\begin{array}{c}0.039 \\
(0.004)\end{array}$ & $\begin{array}{c}0.080 \\
(0.004)\end{array}$ & $\begin{array}{c}0.174 \\
(0.004)\end{array}$ & $\begin{array}{c}0.210 \\
(0.043)\end{array}$ & $\begin{array}{c}1.230 \\
(0.062)\end{array}$ & $\begin{array}{c}1.540 \\
(0.048)\end{array}$ & $\begin{array}{l}10.710 \\
(0.038)\end{array}$ & $\begin{array}{c}8.711 \\
(1.307)\end{array}$ & $\begin{array}{c}9.670 \\
(0.188)\end{array}$ & $\begin{array}{c}6.828 \\
(2.048)\end{array}$ & $\begin{array}{l}\ldots \\
(\ldots)\end{array}$ & $\begin{array}{c}0.039 \\
(0.008)\end{array}$ & $\begin{array}{l}\ldots \\
(\ldots)\end{array}$ & $\begin{array}{c}0.049 \\
(0.001)\end{array}$ \\
\hline IRAS F01364-1042 & $\begin{array}{c}0.003 \\
(0.000)\end{array}$ & $\begin{array}{c}0.004 \\
(0.000)\end{array}$ & $\begin{array}{c}0.007 \\
(0.000)\end{array}$ & $\begin{array}{c}0.021 \\
(0.000)\end{array}$ & $\begin{array}{r}<0.16 \\
(\ldots)\end{array}$ & $\begin{array}{c}0.255 \\
(0.013)\end{array}$ & $\begin{array}{c}0.440 \\
(0.036)\end{array}$ & $\begin{array}{c}6.620 \\
(0.042)\end{array}$ & $\begin{array}{c}5.986 \\
(0.898)\end{array}$ & $\begin{array}{c}6.880 \\
(0.114)\end{array}$ & $\begin{array}{c}3.660 \\
(1.098)\end{array}$ & $\begin{array}{l}\ldots \\
(\ldots)\end{array}$ & $\begin{array}{l}\ldots \\
(\ldots)\end{array}$ & $\begin{array}{c}0.012 \\
(\ldots)\end{array}$ & $\begin{array}{c}0.015 \\
(0.001)\end{array}$ \\
\hline III Zw 035 & $\begin{array}{c}0.018 \\
(0.002)\end{array}$ & $\begin{array}{c}0.013 \\
(0.001)\end{array}$ & $\begin{array}{c}0.026 \\
(0.002)\end{array}$ & $\begin{array}{c}0.062 \\
(0.002)\end{array}$ & $\begin{array}{r}<0.06 \\
(\ldots)\end{array}$ & $\begin{array}{c}0.761 \\
(0.038)\end{array}$ & $\begin{array}{c}1.030 \\
(0.059)\end{array}$ & $\begin{array}{l}13.250 \\
(0.050)\end{array}$ & $\begin{array}{l}10.962 \\
(1.644)\end{array}$ & $\begin{array}{l}14.300 \\
(0.155)\end{array}$ & $\begin{array}{c}7.317 \\
(2.195)\end{array}$ & $\begin{array}{l}\ldots \\
(\ldots)\end{array}$ & $\begin{array}{c}0.076 \\
(0.015)\end{array}$ & $\begin{array}{l}\ldots \\
(\ldots)\end{array}$ & $\begin{array}{c}0.041 \\
(0.001)\end{array}$ \\
\hline NGC 0695 & $\begin{array}{c}0.058 \\
(0.006)\end{array}$ & $\begin{array}{c}0.042 \\
(0.004)\end{array}$ & $\begin{array}{c}0.125 \\
(0.006)\end{array}$ & $\begin{array}{c}0.362 \\
(0.006)\end{array}$ & $\begin{array}{c}0.500 \\
(0.023)\end{array}$ & $\begin{array}{c}0.722 \\
(0.036)\end{array}$ & $\begin{array}{c}0.830 \\
(0.041)\end{array}$ & $\begin{array}{c}7.590 \\
(0.031)\end{array}$ & $\begin{array}{c}8.828 \\
(1.324)\end{array}$ & $\begin{array}{l}13.560 \\
(0.167)\end{array}$ & $\begin{array}{l}11.200 \\
(3.360)\end{array}$ & $\begin{array}{l}\ldots \\
(\ldots)\end{array}$ & $\begin{array}{c}0.136 \\
(0.021)\end{array}$ & $\begin{array}{l}\ldots \\
(\ldots)\end{array}$ & $\begin{array}{c}0.066 \\
(0.001)\end{array}$ \\
\hline NGC 0958 & $\begin{array}{c}0.128 \\
(0.013)\end{array}$ & $\begin{array}{c}0.082 \\
(0.008)\end{array}$ & $\begin{array}{c}0.189 \\
(0.013)\end{array}$ & $\begin{array}{c}0.421 \\
(0.013)\end{array}$ & $\begin{array}{c}0.620 \\
(0.030)\end{array}$ & $\begin{array}{c}0.516 \\
(0.026)\end{array}$ & $\begin{array}{c}0.940 \\
(0.035)\end{array}$ & $\begin{array}{c}5.850 \\
(0.040)\end{array}$ & $\begin{array}{c}7.846 \\
(1.177)\end{array}$ & $\begin{array}{l}15.080 \\
(0.198)\end{array}$ & $\begin{array}{l}19.138 \\
(5.741)\end{array}$ & $\begin{array}{c}2.251 \\
(0.428)\end{array}$ & $\begin{array}{c}0.262 \\
(0.034)\end{array}$ & $\begin{array}{l}\ldots \\
(\ldots)\end{array}$ & $\begin{array}{c}0.058 \\
(0.001)\end{array}$ \\
\hline UGC 02238 & $\begin{array}{c}0.050 \\
(0.005)\end{array}$ & $\begin{array}{c}0.038 \\
(0.004)\end{array}$ & $\begin{array}{c}0.142 \\
(0.005)\end{array}$ & $\begin{array}{c}0.383 \\
(0.005)\end{array}$ & $\begin{array}{c}0.360 \\
(0.030)\end{array}$ & $\begin{array}{c}0.524 \\
(0.026)\end{array}$ & $\begin{array}{c}0.650 \\
(0.042)\end{array}$ & $\begin{array}{c}8.170 \\
(0.036)\end{array}$ & $\begin{array}{c}9.526 \\
(1.429)\end{array}$ & $\begin{array}{l}15.670 \\
(0.128)\end{array}$ & $\begin{array}{l}13.240 \\
(3.972)\end{array}$ & $\begin{array}{l}\ldots \\
(\ldots)\end{array}$ & $\begin{array}{c}0.104 \\
(0.014)\end{array}$ & $\begin{array}{l}\ldots \\
(\ldots)\end{array}$ & $\begin{array}{c}0.067 \\
(0.001)\end{array}$ \\
\hline UGC 02369 & $\begin{array}{c}0.042 \\
(0.004)\end{array}$ & $\begin{array}{l}0.030 \\
(0.003)\end{array}$ & $\begin{array}{c}0.063 \\
(0.004)\end{array}$ & $\begin{array}{c}0.149 \\
(0.004)\end{array}$ & $\begin{array}{c}0.230 \\
(0.022)\end{array}$ & $\begin{array}{c}1.160 \\
(0.058)\end{array}$ & $\begin{array}{c}1.880 \\
(0.042)\end{array}$ & $\begin{array}{c}8.070 \\
(0.043)\end{array}$ & $\begin{array}{c}7.823 \\
(1.173)\end{array}$ & $\begin{array}{l}11.180 \\
(0.190)\end{array}$ & $\begin{array}{c}8.717 \\
(2.615)\end{array}$ & $\begin{array}{c}0.523 \\
(0.120)\end{array}$ & $\begin{array}{c}0.072 \\
(0.013)\end{array}$ & $\begin{array}{l}\ldots \\
(\ldots)\end{array}$ & $\begin{array}{c}0.050 \\
(0.001)\end{array}$ \\
\hline IRAS F03359+1523 & $\begin{array}{c}0.010 \\
(0.001)\end{array}$ & $\begin{array}{c}0.008 \\
(0.001)\end{array}$ & $\begin{array}{c}0.020 \\
(0.001)\end{array}$ & $\begin{array}{c}0.053 \\
(0.001)\end{array}$ & $\begin{array}{r}<0.07 \\
(\ldots)\end{array}$ & $\begin{array}{c}0.428 \\
(0.021)\end{array}$ & $\begin{array}{c}0.650 \\
(0.043)\end{array}$ & $\begin{array}{c}5.970 \\
(0.041)\end{array}$ & $\begin{array}{c}6.409 \\
(0.961)\end{array}$ & $\begin{array}{c}7.270 \\
(0.128)\end{array}$ & $\begin{array}{c}3.652 \\
(1.096)\end{array}$ & $\begin{array}{l}\ldots \\
(\ldots)\end{array}$ & $\begin{array}{c}0.044 \\
(0.009)\end{array}$ & $\begin{array}{l}\ldots \\
(\ldots)\end{array}$ & $\begin{array}{c}0.019 \\
(0.001)\end{array}$ \\
\hline UGC 02982 & $\begin{array}{c}0.076 \\
(0.008)\end{array}$ & $\begin{array}{c}0.053 \\
(0.005)\end{array}$ & $\begin{array}{c}0.186 \\
(0.008)\end{array}$ & $\begin{array}{c}0.481 \\
(0.008)\end{array}$ & $\begin{array}{c}0.570 \\
(0.014)\end{array}$ & $\begin{array}{c}0.673 \\
(0.034)\end{array}$ & $\begin{array}{c}0.830 \\
(0.019)\end{array}$ & $\begin{array}{c}8.390 \\
(0.043)\end{array}$ & $\begin{array}{c}9.862 \\
(1.479)\end{array}$ & $\begin{array}{c}16.820 \\
(0.287)\end{array}$ & $\begin{array}{l}15.831 \\
(4.749)\end{array}$ & $\begin{array}{l}\ldots \\
(\ldots)\end{array}$ & $\begin{array}{c}0.176 \\
(0.034)\end{array}$ & $\begin{array}{l}\ldots \\
(\ldots)\end{array}$ & $\begin{array}{c}0.082 \\
(0.001)\end{array}$ \\
\hline ESO 550-IG025 & $\begin{array}{c}0.029 \\
(0.003)\end{array}$ & $\begin{array}{c}0.021 \\
(0.002)\end{array}$ & $\begin{array}{c}0.045 \\
(0.003)\end{array}$ & $\begin{array}{c}0.131 \\
(0.003)\end{array}$ & $\begin{array}{c}0.220 \\
(0.024)\end{array}$ & $\begin{array}{c}0.392 \\
(0.020)\end{array}$ & $\begin{array}{c}0.510 \\
(0.034)\end{array}$ & $\begin{array}{c}5.690 \\
(0.030)\end{array}$ & $\begin{array}{c}6.456 \\
(0.968)\end{array}$ & $\begin{array}{c}9.470 \\
(0.115)\end{array}$ & $\begin{array}{l}7.724 \\
(2.317)\end{array}$ & $\begin{array}{l}\ldots \\
(\ldots)\end{array}$ & $\begin{array}{l}\ldots \\
(\ldots)\end{array}$ & $\begin{array}{l}\ldots \\
(\ldots)\end{array}$ & $\begin{array}{c}0.038 \\
(0.001)\end{array}$ \\
\hline NGC 1614 & $\begin{array}{c}0.101 \\
(0.010)\end{array}$ & $\begin{array}{c}0.076 \\
(0.008)\end{array}$ & $\begin{array}{c}0.279 \\
(0.010)\end{array}$ & $\begin{array}{c}0.742 \\
(0.010)\end{array}$ & $\begin{array}{c}1.380 \\
(0.023)\end{array}$ & $\begin{array}{c}6.552 \\
(0.328)\end{array}$ & $\begin{array}{c}7.500 \\
(0.025)\end{array}$ & $\begin{array}{c}32.120 \\
(0.083)\end{array}$ & $\begin{array}{c}26.535 \\
(3.980)\end{array}$ & $\begin{array}{c}34.320 \\
(0.430)\end{array}$ & $\begin{array}{l}18.674 \\
(5.602)\end{array}$ & $\begin{array}{c}0.981 \\
(0.167)\end{array}$ & $\begin{array}{c}0.140 \\
(0.020)\end{array}$ & $\begin{array}{c}0.063 \\
(\ldots)\end{array}$ & $\begin{array}{c}0.123 \\
(0.001)\end{array}$ \\
\hline IRAS F05189-2524 & $\begin{array}{c}0.100 \\
(0.010)\end{array}$ & $\begin{array}{c}0.129 \\
(0.013)\end{array}$ & $\begin{array}{c}0.227 \\
(0.010)\end{array}$ & $\begin{array}{c}0.290 \\
(0.010)\end{array}$ & $\begin{array}{c}0.740 \\
(0.016)\end{array}$ & $\begin{array}{c}2.546 \\
(0.127)\end{array}$ & $\begin{array}{c}3.470 \\
(0.018)\end{array}$ & $\begin{array}{l}13.250 \\
(0.029)\end{array}$ & $\begin{array}{l}\ldots \\
(\ldots)\end{array}$ & $\begin{array}{l}11.840 \\
(0.077)\end{array}$ & $\begin{array}{l}\ldots \\
(\ldots)\end{array}$ & $\begin{array}{l}\ldots \\
(\ldots)\end{array}$ & $\begin{array}{l}\ldots \\
(\ldots)\end{array}$ & $\begin{array}{l}\ldots \\
(\ldots)\end{array}$ & $\begin{array}{c}0.028 \\
(0.001)\end{array}$ \\
\hline NGC 2623 & $\begin{array}{c}0.031 \\
(0.003)\end{array}$ & $\begin{array}{c}0.027 \\
(0.003)\end{array}$ & $\begin{array}{c}0.056 \\
(0.003)\end{array}$ & $\begin{array}{c}0.140 \\
(0.003)\end{array}$ & $\begin{array}{c}0.210 \\
(0.023)\end{array}$ & $\begin{array}{c}1.399 \\
(0.070)\end{array}$ & $\begin{array}{l}1.810 \\
(0.041)\end{array}$ & $\begin{array}{c}23.740 \\
(0.028)\end{array}$ & $\begin{array}{c}20.912 \\
(3.137)\end{array}$ & $\begin{array}{c}25.880 \\
(0.111)\end{array}$ & $\begin{array}{l}14.424 \\
(4.327)\end{array}$ & $\begin{array}{l}\ldots \\
(\ldots)\end{array}$ & $\begin{array}{c}0.091 \\
(0.014)\end{array}$ & $\begin{array}{c}0.057 \\
(0.009)\end{array}$ & $\begin{array}{c}0.099 \\
(0.001)\end{array}$ \\
\hline IRAS F08572+3915 & $\begin{array}{c}0.041 \\
(0.004)\end{array}$ & $\begin{array}{c}0.101 \\
(0.004)\end{array}$ & $\begin{array}{c}0.297 \\
(0.004)\end{array}$ & $\begin{array}{c}0.314 \\
(0.004)\end{array}$ & $\begin{array}{c}0.330 \\
(0.031)\end{array}$ & $\begin{array}{c}1.444 \\
(0.072)\end{array}$ & $\begin{array}{c}1.760 \\
(0.033)\end{array}$ & $\begin{array}{c}7.300 \\
(0.028)\end{array}$ & $\begin{array}{c}5.613 \\
(0.842)\end{array}$ & $\begin{array}{c}4.770 \\
(0.152)\end{array}$ & $\begin{array}{c}2.442 \\
(0.733)\end{array}$ & $\begin{array}{l}\ldots \\
(\ldots)\end{array}$ & $\begin{array}{c}0.017 \\
(0.007)\end{array}$ & $\begin{array}{l}\ldots \\
(\ldots)\end{array}$ & $\begin{array}{c}0.005 \\
(0.001)\end{array}$ \\
\hline UGC 04881 & $\begin{array}{c}0.017 \\
(0.002)\end{array}$ & $\begin{array}{c}0.009 \\
(0.001)\end{array}$ & $\begin{array}{c}0.008 \\
(0.002)\end{array}$ & $\begin{array}{c}0.004 \\
(0.002)\end{array}$ & $\begin{array}{c}0.140 \\
(0.037)\end{array}$ & $\begin{array}{c}0.435 \\
(0.022)\end{array}$ & $\begin{array}{c}0.610 \\
(0.034)\end{array}$ & $\begin{array}{c}6.070 \\
(0.048)\end{array}$ & $\begin{array}{c}6.995 \\
(1.049)\end{array}$ & $\begin{array}{l}10.330 \\
(0.109)\end{array}$ & $\begin{array}{c}8.447 \\
(2.534)\end{array}$ & $\begin{array}{l}\ldots \\
(\ldots)\end{array}$ & $\begin{array}{c}0.065 \\
(0.013)\end{array}$ & $\begin{array}{l}\ldots \\
(\ldots)\end{array}$ & $\begin{array}{c}0.032 \\
(0.001)\end{array}$ \\
\hline UGC 05101 & $\begin{array}{c}0.046 \\
(0.005)\end{array}$ & $\begin{array}{c}0.078 \\
(0.008)\end{array}$ & $\begin{array}{c}0.096 \\
(0.005)\end{array}$ & $\begin{array}{c}0.144 \\
(0.005)\end{array}$ & $\begin{array}{c}0.250 \\
(0.027)\end{array}$ & $\begin{array}{c}0.808 \\
(0.040)\end{array}$ & $\begin{array}{l}1.020 \\
(0.027)\end{array}$ & $\begin{array}{l}11.680 \\
(0.034)\end{array}$ & $\begin{array}{l}13.195 \\
(1.979)\end{array}$ & $\begin{array}{l}19.910 \\
(0.137)\end{array}$ & $\begin{array}{l}13.393 \\
(4.018)\end{array}$ & $\begin{array}{l}\ldots \\
(\ldots)\end{array}$ & $\begin{array}{l}\ldots \\
(\ldots)\end{array}$ & $\begin{array}{c}0.076 \\
(0.011)\end{array}$ & $\begin{array}{c}0.150 \\
(0.001)\end{array}$ \\
\hline $\mathrm{MCG}+08-18-013$ & $\begin{array}{c}0.024 \\
(0.002)\end{array}$ & $\begin{array}{l}0.017 \\
(0.002)\end{array}$ & $\begin{array}{c}0.047 \\
(0.002)\end{array}$ & $\begin{array}{c}0.126 \\
(0.002)\end{array}$ & $\begin{array}{c}0.100 \\
(0.019)\end{array}$ & $\begin{array}{c}0.587 \\
(0.029)\end{array}$ & $\begin{array}{c}0.750 \\
(0.028)\end{array}$ & $\begin{array}{c}5.680 \\
(0.028)\end{array}$ & $\begin{array}{c}7.316 \\
(1.097)\end{array}$ & $\begin{array}{c}8.420 \\
(0.143)\end{array}$ & $\begin{array}{c}6.722 \\
(2.017)\end{array}$ & $\begin{array}{l}\ldots \\
(\ldots)\end{array}$ & $\begin{array}{c}0.042 \\
(0.010)\end{array}$ & $\begin{array}{l}\ldots \\
(\ldots)\end{array}$ & $\begin{array}{c}0.022 \\
(0.001)\end{array}$ \\
\hline IC $0563 / 4$ & $\begin{array}{c}0.069 \\
(0.007)\end{array}$ & $\begin{array}{l}0.046 \\
(0.005)\end{array}$ & $\begin{array}{l}0.123 \\
(0.007)\end{array}$ & $\begin{array}{c}0.307 \\
(0.007)\end{array}$ & $\begin{array}{c}0.460 \\
(0.021)\end{array}$ & $\begin{array}{c}0.490 \\
(0.025)\end{array}$ & $\begin{array}{c}0.540 \\
(0.039)\end{array}$ & $\begin{array}{c}5.260 \\
(0.026)\end{array}$ & $\begin{array}{c}7.237 \\
(1.086)\end{array}$ & $\begin{array}{l}12.180 \\
(0.058)\end{array}$ & $\begin{array}{l}18.104 \\
(5.431)\end{array}$ & $\begin{array}{l}\ldots \\
(\ldots)\end{array}$ & $\begin{array}{l}0.228 \\
(0.035)\end{array}$ & $\begin{array}{l}\ldots \\
(\ldots)\end{array}$ & $\begin{array}{c}0.060 \\
(0.001)\end{array}$ \\
\hline
\end{tabular}


Table 5

\begin{tabular}{|c|c|c|c|c|c|c|c|c|c|c|c|c|c|c|c|}
\hline Object & $\begin{array}{l}\text { IRAC1 } \\
3.6 \mu \mathrm{m} \\
(2)\end{array}$ & $\begin{array}{l}\text { IRAC2 } \\
4.5 \mu \mathrm{m} \\
(3)\end{array}$ & $\begin{array}{l}\text { IRAC3 } \\
5.8 \mu \mathrm{m} \\
\text { (4) }\end{array}$ & $\begin{array}{l}\text { IRAC4 } \\
8.0 \mu \mathrm{m} \\
(5)\end{array}$ & $\begin{array}{c}\text { IRAS1 } \\
12 \mu \mathrm{m} \\
\text { (6) }\end{array}$ & $\begin{array}{l}\text { MIPS1 } \\
24 \mu \mathrm{m} \\
\quad(7)\end{array}$ & $\begin{array}{l}\text { IRAS2 } \\
25 \mu \mathrm{m} \\
(8)\end{array}$ & $\begin{array}{l}\text { IRAS3 } \\
60 \mu \mathrm{m} \\
\quad(9)\end{array}$ & $\begin{array}{l}\text { MIPS2 } \\
70 \mu \mathrm{m} \\
(10)\end{array}$ & $\begin{array}{c}\text { IRAS4 } \\
100 \mu \mathrm{m} \\
(11)\end{array}$ & $\begin{array}{l}\text { MIPS3 } \\
160 \mu \mathrm{m} \\
(12)\end{array}$ & $\begin{array}{c}\text { SCUBA1 } \\
450 \mu \mathrm{m} \\
(13)\end{array}$ & $\begin{array}{c}\text { SCUBA2 } \\
850 \mu \mathrm{m} \\
(14)\end{array}$ & $\begin{array}{c}\text { VLA1 } \\
4.85 \mathrm{GHz} \\
(15)\end{array}$ & $\begin{array}{c}\text { VLA2 } \\
1.49 \mathrm{GHz} \\
(16)\end{array}$ \\
\hline NGC 3110 & $\begin{array}{c}0.101 \\
(0.010)\end{array}$ & $\begin{array}{c}0.069 \\
(0.007)\end{array}$ & $\begin{array}{c}0.219 \\
(0.010)\end{array}$ & $\begin{array}{c}0.555 \\
(0.010)\end{array}$ & $\begin{array}{c}0.590 \\
(0.035)\end{array}$ & $\begin{array}{l}1.016 \\
(0.051)\end{array}$ & $\begin{array}{l}1.130 \\
(0.048)\end{array}$ & $\begin{array}{l}11.280 \\
(0.033)\end{array}$ & $\begin{array}{l}11.779 \\
(1.767)\end{array}$ & $\begin{array}{l}22.270 \\
(0.085)\end{array}$ & $\begin{array}{l}17.168 \\
(5.150)\end{array}$ & $(\ldots)$ & $\begin{array}{c}0.188 \\
(0.028)\end{array}$ & $\begin{array}{l}\ldots \\
(\ldots)\end{array}$ & $\begin{array}{c}0.109 \\
(0.001)\end{array}$ \\
\hline IRAS F10173+0828 & $\begin{array}{c}0.011 \\
(0.001)\end{array}$ & $\begin{array}{c}0.006 \\
(0.001)\end{array}$ & $\begin{array}{c}0.013 \\
(0.001)\end{array}$ & $\begin{array}{c}0.014 \\
(0.001)\end{array}$ & $\begin{array}{c}0.190 \\
(0.029)\end{array}$ & $\begin{array}{c}0.276 \\
(0.014)\end{array}$ & $\begin{array}{c}0.550 \\
(0.049)\end{array}$ & $\begin{array}{c}5.610 \\
(0.025)\end{array}$ & $\begin{array}{c}5.241 \\
(0.786)\end{array}$ & $\begin{array}{c}5.860 \\
(0.100)\end{array}$ & $\begin{array}{c}3.264 \\
(0.979)\end{array}$ & $\begin{array}{l}\ldots \\
(\ldots)\end{array}$ & $\begin{array}{c}0.036 \\
(0.006)\end{array}$ & $\begin{array}{l}\ldots \\
(\ldots)\end{array}$ & $\begin{array}{c}0.009 \\
(0.001)\end{array}$ \\
\hline IRAS F10565+2448 & $\begin{array}{c}0.021 \\
(0.002)\end{array}$ & $\begin{array}{c}0.018 \\
(0.002)\end{array}$ & $\begin{array}{c}0.039 \\
(0.002)\end{array}$ & $\begin{array}{c}0.127 \\
(0.002)\end{array}$ & $\begin{array}{c}0.200 \\
(0.030)\end{array}$ & $\begin{array}{c}0.976 \\
(0.049)\end{array}$ & $\begin{array}{c}1.270 \\
(0.031)\end{array}$ & $\begin{array}{l}12.100 \\
(0.025)\end{array}$ & $\begin{array}{l}11.259 \\
(1.689)\end{array}$ & $\begin{array}{l}15.010 \\
(0.122)\end{array}$ & $\begin{array}{c}9.174 \\
(2.752)\end{array}$ & $\begin{array}{c}0.533 \\
(\ldots)\end{array}$ & $\begin{array}{c}0.061 \\
(0.013)\end{array}$ & $\begin{array}{l}\ldots \\
(\ldots)\end{array}$ & $\begin{array}{c}0.051 \\
(0.001)\end{array}$ \\
\hline MCG +07-23-019 & $\begin{array}{c}0.016 \\
(0.002)\end{array}$ & $\begin{array}{c}0.012 \\
(0.001)\end{array}$ & $\begin{array}{c}0.034 \\
(0.002)\end{array}$ & $\begin{array}{c}0.093 \\
(0.002)\end{array}$ & $\begin{array}{c}0.200 \\
(0.019)\end{array}$ & $\begin{array}{c}0.245 \\
(0.012)\end{array}$ & $\begin{array}{c}0.710 \\
(0.022)\end{array}$ & $\begin{array}{c}6.380 \\
(0.034)\end{array}$ & $\begin{array}{c}7.172 \\
(1.076)\end{array}$ & $\begin{array}{l}10.300 \\
(0.106)\end{array}$ & $\begin{array}{l}7.284 \\
(2.185)\end{array}$ & $\begin{array}{c}0.646 \\
(0.156)\end{array}$ & $\begin{array}{c}0.092 \\
(0.020)\end{array}$ & $\begin{array}{l}\ldots \\
(\ldots)\end{array}$ & $\begin{array}{c}0.031 \\
(0.001)\end{array}$ \\
\hline CGCG 011-076 & $\begin{array}{c}0.040 \\
(0.004)\end{array}$ & $\begin{array}{c}0.034 \\
(0.003)\end{array}$ & $\begin{array}{c}0.075 \\
(0.004)\end{array}$ & $\begin{array}{c}0.192 \\
(0.004)\end{array}$ & $\begin{array}{c}0.480 \\
(0.039)\end{array}$ & $\begin{array}{c}0.680 \\
(0.034)\end{array}$ & $\begin{array}{c}0.760 \\
(0.050)\end{array}$ & $\begin{array}{c}5.850 \\
(0.057)\end{array}$ & $\begin{array}{c}6.328 \\
(0.949)\end{array}$ & $\begin{array}{c}9.180 \\
(0.253)\end{array}$ & $\begin{array}{c}8.364 \\
(2.509)\end{array}$ & $\begin{array}{c}0.571 \\
(0.166)\end{array}$ & $\begin{array}{c}0.084 \\
(0.013)\end{array}$ & $\begin{array}{l}\ldots \\
(\ldots)\end{array}$ & $\begin{array}{c}0.032 \\
(0.001)\end{array}$ \\
\hline IC 2810 & $\begin{array}{c}0.026 \\
(0.003)\end{array}$ & $\begin{array}{c}0.019 \\
(0.002)\end{array}$ & $\begin{array}{c}0.047 \\
(0.003)\end{array}$ & $\begin{array}{c}0.113 \\
(0.003)\end{array}$ & $\begin{array}{c}0.140 \\
(0.034)\end{array}$ & $\begin{array}{c}0.492 \\
(0.025)\end{array}$ & $\begin{array}{c}0.620 \\
(0.062)\end{array}$ & $\begin{array}{c}6.200 \\
(0.038)\end{array}$ & $\begin{array}{c}8.771 \\
(1.316)\end{array}$ & $\begin{array}{l}10.390 \\
(0.136)\end{array}$ & $\begin{array}{l}12.389 \\
(3.717)\end{array}$ & $\begin{array}{l}\ldots \\
(\ldots)\end{array}$ & $\begin{array}{c}0.106 \\
(0.018)\end{array}$ & $\begin{array}{l}\ldots \\
(\ldots)\end{array}$ & $\begin{array}{c}0.026 \\
(0.001)\end{array}$ \\
\hline NGC 3690/IC 694 & $\begin{array}{c}0.313 \\
(0.031)\end{array}$ & $\begin{array}{c}0.340 \\
(0.034)\end{array}$ & $\begin{array}{c}1.049 \\
(0.031)\end{array}$ & $\begin{array}{c}2.157 \\
(0.031)\end{array}$ & $\begin{array}{c}3.970 \\
(0.020)\end{array}$ & $\begin{array}{l}\ldots \\
(\ldots)\end{array}$ & $\begin{array}{l}24.510 \\
(0.031)\end{array}$ & $\begin{array}{r}113.050 \\
(0.052)\end{array}$ & $\begin{array}{c}79.355 \\
(11.903)\end{array}$ & $\begin{array}{r}111.420 \\
(0.133)\end{array}$ & $\begin{array}{l}\ldots \\
(\ldots)\end{array}$ & $\begin{array}{l}\ldots \\
(\ldots)\end{array}$ & $\begin{array}{l}\ldots \\
(\ldots)\end{array}$ & $\begin{array}{l}\ldots \\
(\ldots)\end{array}$ & $\begin{array}{c}0.658 \\
(0.001)\end{array}$ \\
\hline IRAS F12112+0305 & $\begin{array}{c}0.009 \\
(0.001)\end{array}$ & $\begin{array}{c}0.006 \\
(0.001)\end{array}$ & $\begin{array}{c}0.009 \\
(0.001)\end{array}$ & $\begin{array}{c}0.050 \\
(0.001)\end{array}$ & $\begin{array}{r}<0.11 \\
(\ldots)\end{array}$ & $\begin{array}{c}0.364 \\
(0.018)\end{array}$ & $\begin{array}{c}0.660 \\
(0.054)\end{array}$ & $\begin{array}{c}8.180 \\
(0.032)\end{array}$ & $\begin{array}{l}\ldots \\
(\ldots)\end{array}$ & $\begin{array}{l}9.460 \\
(0.123)\end{array}$ & $\begin{array}{l}\ldots \\
(\ldots)\end{array}$ & $\begin{array}{c}0.429 \\
(\ldots)\end{array}$ & $\begin{array}{c}0.049 \\
(0.010)\end{array}$ & $\begin{array}{l}\ldots \\
(\ldots)\end{array}$ & $\begin{array}{c}0.024 \\
(0.001)\end{array}$ \\
\hline IRAS F12224-0624 & $\begin{array}{c}0.007 \\
(0.001)\end{array}$ & $\begin{array}{c}0.004 \\
(0.000)\end{array}$ & $\begin{array}{c}0.008 \\
(0.001)\end{array}$ & $\begin{array}{c}0.016 \\
(0.001)\end{array}$ & $\begin{array}{r}<0.11 \\
(\ldots)\end{array}$ & $\begin{array}{c}0.159 \\
(0.008)\end{array}$ & $\begin{array}{c}0.200 \\
(0.044)\end{array}$ & $\begin{array}{c}5.990 \\
(0.044)\end{array}$ & $\begin{array}{c}5.832 \\
(0.875)\end{array}$ & $\begin{array}{c}8.130 \\
(0.139)\end{array}$ & $\begin{array}{c}4.798 \\
(1.439)\end{array}$ & $\begin{array}{l}\ldots \\
(\ldots)\end{array}$ & $\begin{array}{c}0.074 \\
(0.015)\end{array}$ & $\begin{array}{l}\ldots \\
(\ldots)\end{array}$ & $\begin{array}{c}0.009 \\
(0.001)\end{array}$ \\
\hline UGC 08058 & $\begin{array}{c}0.206 \\
(0.021)\end{array}$ & $\begin{array}{c}0.280 \\
(0.028)\end{array}$ & $\begin{array}{c}1.394 \\
(0.021)\end{array}$ & $\begin{array}{c}0.907 \\
(0.021)\end{array}$ & $\begin{array}{c}1.830 \\
(0.017)\end{array}$ & $\begin{array}{c}4.337 \\
(0.217)\end{array}$ & $\begin{array}{c}8.840 \\
(0.028)\end{array}$ & $\begin{array}{l}30.800 \\
(0.042)\end{array}$ & $\begin{array}{l}\ldots \\
(\ldots)\end{array}$ & $\begin{array}{l}29.740 \\
(0.108)\end{array}$ & $\begin{array}{l}\ldots \\
(\ldots)\end{array}$ & $\begin{array}{l}\ldots \\
(\ldots)\end{array}$ & $\begin{array}{l}\ldots \\
(\ldots)\end{array}$ & $\begin{array}{c}0.414 \\
(0.062)\end{array}$ & $\begin{array}{c}0.296 \\
(0.001)\end{array}$ \\
\hline NGC 4922 & $\begin{array}{c}0.059 \\
(0.006)\end{array}$ & $\begin{array}{c}0.051 \\
(0.005)\end{array}$ & $\begin{array}{l}0.068 \\
(0.006)\end{array}$ & $\begin{array}{c}0.132 \\
(0.006)\end{array}$ & $\begin{array}{c}0.270 \\
(0.035)\end{array}$ & $\begin{array}{l}1.171 \\
(0.059)\end{array}$ & $\begin{array}{c}1.480 \\
(0.030)\end{array}$ & $\begin{array}{c}6.210 \\
(0.040)\end{array}$ & $\begin{array}{c}5.740 \\
(0.861)\end{array}$ & $\begin{array}{c}7.330 \\
(0.093)\end{array}$ & $\begin{array}{c}5.256 \\
(1.577)\end{array}$ & $\begin{array}{l}\ldots \\
(\ldots)\end{array}$ & $\begin{array}{c}0.053 \\
(0.012)\end{array}$ & $\begin{array}{l}\ldots \\
(\ldots)\end{array}$ & $\begin{array}{c}0.038 \\
(0.001)\end{array}$ \\
\hline ESO 507-G070 & $\begin{array}{c}0.037 \\
(0.004)\end{array}$ & $\begin{array}{c}0.026 \\
(0.003)\end{array}$ & $\begin{array}{c}0.047 \\
(0.004)\end{array}$ & $\begin{array}{c}0.099 \\
(0.004)\end{array}$ & $\begin{array}{c}0.250 \\
(0.020)\end{array}$ & $\begin{array}{c}0.643 \\
(0.032)\end{array}$ & $\begin{array}{c}0.800 \\
(0.046)\end{array}$ & $\begin{array}{l}13.040 \\
(0.051)\end{array}$ & $\begin{array}{l}13.102 \\
(1.965)\end{array}$ & $\begin{array}{l}15.710 \\
(0.159)\end{array}$ & $\begin{array}{c}9.489 \\
(2.847)\end{array}$ & $\begin{array}{l}\ldots \\
(\ldots)\end{array}$ & . & $\ldots$ & $0.060^{\mathrm{a}}$ \\
\hline IC 0860 & $\begin{array}{c}0.023 \\
(0.002)\end{array}$ & $\begin{array}{c}0.014 \\
(0.001)\end{array}$ & $\begin{array}{c}0.018 \\
(0.002)\end{array}$ & $\begin{array}{c}0.036 \\
(0.002)\end{array}$ & $\begin{array}{r}<0.14 \\
(\ldots)\end{array}$ & $\begin{array}{c}0.901 \\
(0.045)\end{array}$ & $\begin{array}{c}1.340 \\
(0.044)\end{array}$ & $\begin{array}{l}18.610 \\
(0.071)\end{array}$ & $\begin{array}{l}15.379 \\
(2.307)\end{array}$ & $\begin{array}{l}18.660 \\
(0.900)\end{array}$ & $\begin{array}{c}9.336 \\
(2.801)\end{array}$ & $\begin{array}{l}\ldots \\
(\ldots)\end{array}$ & $\begin{array}{c}0.118 \\
(0.020)\end{array}$ & $\begin{array}{l}\ldots \\
(\ldots)\end{array}$ & $\begin{array}{c}0.033 \\
(0.001)\end{array}$ \\
\hline VV 250a & $\begin{array}{c}0.033 \\
(0.003)\end{array}$ & $\begin{array}{c}0.026 \\
(0.003)\end{array}$ & $\begin{array}{c}0.085 \\
(0.003)\end{array}$ & $\begin{array}{c}0.213 \\
(0.003)\end{array}$ & $\begin{array}{c}0.350 \\
(0.022)\end{array}$ & $\begin{array}{l}\ldots \\
(\ldots)\end{array}$ & $\begin{array}{c}1.950 \\
(0.020)\end{array}$ & $\begin{array}{l}11.390 \\
(0.035)\end{array}$ & $\begin{array}{l}\ldots \\
(\ldots)\end{array}$ & $\begin{array}{l}12.410 \\
(0.093)\end{array}$ & $\begin{array}{l}\ldots \\
(\ldots)\end{array}$ & $\begin{array}{l}\ldots \\
(\ldots)\end{array}$ & $\begin{array}{l}\ldots \\
(\ldots)\end{array}$ & $\begin{array}{l}\ldots \\
(\ldots)\end{array}$ & $\begin{array}{c}0.050 \\
(0.001)\end{array}$ \\
\hline UGC 08387 & $\begin{array}{c}0.032 \\
(0.003)\end{array}$ & $\begin{array}{c}0.025 \\
(0.003)\end{array}$ & $\begin{array}{c}0.083 \\
(0.003)\end{array}$ & $\begin{array}{c}0.219 \\
(0.003)\end{array}$ & $\begin{array}{c}0.250 \\
(0.029)\end{array}$ & $\begin{array}{l}1.070 \\
(0.054)\end{array}$ & $\begin{array}{c}1.420 \\
(0.040)\end{array}$ & $\begin{array}{l}17.040 \\
(0.088)\end{array}$ & $\begin{array}{l}17.681 \\
(2.652)\end{array}$ & $\begin{array}{l}24.380 \\
(0.120)\end{array}$ & $\begin{array}{l}14.213 \\
(4.264)\end{array}$ & $\begin{array}{l}\ldots \\
(\ldots)\end{array}$ & $\begin{array}{c}0.113 \\
(0.015)\end{array}$ & $\begin{array}{c}0.053 \\
(0.008)\end{array}$ & $\begin{array}{c}0.101 \\
(0.001)\end{array}$ \\
\hline NGC 5104 & $\begin{array}{c}0.055 \\
(0.006)\end{array}$ & $\begin{array}{c}0.038 \\
(0.004)\end{array}$ & $\begin{array}{c}0.076 \\
(0.006)\end{array}$ & $\begin{array}{c}0.179 \\
(0.006)\end{array}$ & $\begin{array}{c}0.390 \\
(0.034)\end{array}$ & $\begin{array}{c}0.554 \\
(0.028)\end{array}$ & $\begin{array}{c}0.740 \\
(0.091)\end{array}$ & $\begin{array}{c}6.780 \\
(0.049)\end{array}$ & $\begin{array}{c}7.461 \\
(1.119)\end{array}$ & $\begin{array}{l}13.370 \\
(0.103)\end{array}$ & $\begin{array}{l}11.508 \\
(3.452)\end{array}$ & $\begin{array}{l}\ldots \\
(\ldots)\end{array}$ & $\begin{array}{c}0.091 \\
(0.020)\end{array}$ & $\begin{array}{l}\ldots \\
(\ldots)\end{array}$ & $\begin{array}{c}0.035 \\
(0.001)\end{array}$ \\
\hline MCG -03-34-064 & $\begin{array}{c}0.093 \\
(0.009)\end{array}$ & $\begin{array}{c}0.105 \\
(0.011)\end{array}$ & $\begin{array}{c}0.214 \\
(0.009)\end{array}$ & $\begin{array}{c}0.328 \\
(0.009)\end{array}$ & $\begin{array}{c}0.940 \\
(0.040)\end{array}$ & $\begin{array}{c}2.253 \\
(0.113)\end{array}$ & $\begin{array}{c}2.970 \\
(0.045)\end{array}$ & $\begin{array}{c}6.200 \\
(0.040)\end{array}$ & $\begin{array}{c}1.292 \\
(0.194)\end{array}$ & $\begin{array}{c}6.200 \\
(0.143)\end{array}$ & $\begin{array}{c}2.499 \\
(0.750)\end{array}$ & $\begin{array}{l}\ldots \\
(\ldots)\end{array}$ & $\begin{array}{l}\ldots \\
(\ldots)\end{array}$ & $\begin{array}{l}\ldots \\
(\ldots)\end{array}$ & $\begin{array}{l}0.267^{\mathrm{a}} \\
(\ldots)\end{array}$ \\
\hline NGC 5135 & $\begin{array}{c}0.132 \\
(0.013)\end{array}$ & $\begin{array}{c}0.104 \\
(0.010)\end{array}$ & $\begin{array}{c}0.220 \\
(0.013)\end{array}$ & $\begin{array}{c}0.459 \\
(0.013)\end{array}$ & $\begin{array}{c}0.630 \\
(0.035)\end{array}$ & $\begin{array}{l}\ldots \\
(\ldots)\end{array}$ & $\begin{array}{c}2.380 \\
(0.058)\end{array}$ & $\begin{array}{l}16.860 \\
(0.046)\end{array}$ & $\begin{array}{l}\ldots \\
(\ldots)\end{array}$ & $\begin{array}{l}30.970 \\
(0.177)\end{array}$ & $\begin{array}{l}23.012 \\
(6.904)\end{array}$ & $\begin{array}{l}\ldots \\
(\ldots)\end{array}$ & $\begin{array}{l}\ldots \\
(\ldots)\end{array}$ & $\begin{array}{l}\ldots \\
(\ldots)\end{array}$ & $\begin{array}{l}0.194^{\mathrm{a}} \\
(\ldots)\end{array}$ \\
\hline NGC 5256 & $\begin{array}{c}0.043 \\
(0.004)\end{array}$ & $\begin{array}{c}0.033 \\
(0.003)\end{array}$ & $\begin{array}{c}0.067 \\
(0.004)\end{array}$ & $\begin{array}{c}0.161 \\
(0.004)\end{array}$ & $\begin{array}{c}0.320 \\
(0.018)\end{array}$ & $\begin{array}{c}0.873 \\
(0.044)\end{array}$ & $\begin{array}{c}1.070 \\
(0.028)\end{array}$ & $\begin{array}{c}7.250 \\
(0.033)\end{array}$ & $\begin{array}{c}7.746 \\
(1.162)\end{array}$ & $\begin{array}{l}10.110 \\
(0.135)\end{array}$ & $\begin{array}{c}7.021 \\
(2.106)\end{array}$ & $\begin{array}{l}\ldots \\
(\ldots)\end{array}$ & $\begin{array}{c}0.082 \\
(0.017)\end{array}$ & $\begin{array}{c}0.047 \\
(0.007)\end{array}$ & $\begin{array}{c}0.113 \\
(0.001)\end{array}$ \\
\hline NGC 5257/8 & $\begin{array}{c}0.088 \\
(0.009)\end{array}$ & $\begin{array}{c}0.060 \\
(0.006)\end{array}$ & $\begin{array}{c}0.171 \\
(0.009)\end{array}$ & $\begin{array}{c}0.441 \\
(0.009)\end{array}$ & $\begin{array}{c}0.570 \\
(0.031)\end{array}$ & $\begin{array}{l}1.180 \\
(0.059)\end{array}$ & $\begin{array}{c}1.340 \\
(0.069)\end{array}$ & $\begin{array}{l}10.730 \\
(0.059)\end{array}$ & $\begin{array}{l}14.015 \\
(2.102)\end{array}$ & $\begin{array}{l}19.970 \\
(0.050)\end{array}$ & $\begin{array}{l}22.598 \\
(6.779)\end{array}$ & $\begin{array}{l}\ldots \\
(\ldots)\end{array}$ & $\begin{array}{c}0.283 \\
(0.039)\end{array}$ & $\begin{array}{l}\ldots \\
(\ldots)\end{array}$ & $\begin{array}{c}0.076 \\
(0.001)\end{array}$ \\
\hline UGC 08696 & $\begin{array}{c}0.032 \\
(0.003)\end{array}$ & $\begin{array}{c}0.039 \\
(0.004)\end{array}$ & $\begin{array}{c}0.073 \\
(0.003)\end{array}$ & $\begin{array}{c}0.143 \\
(0.003)\end{array}$ & $\begin{array}{l}0.240 \\
(0.017)\end{array}$ & $\begin{array}{c}1.864 \\
(0.093)\end{array}$ & $\begin{array}{c}2.360 \\
(0.021)\end{array}$ & $\begin{array}{l}22.510 \\
(0.042)\end{array}$ & $\begin{array}{l}20.206 \\
(3.031)\end{array}$ & $\begin{array}{l}22.530 \\
(0.070)\end{array}$ & $\begin{array}{l}11.661 \\
(3.498)\end{array}$ & $\begin{array}{l}\ldots \\
(\ldots)\end{array}$ & $\begin{array}{l}\ldots \\
(\ldots)\end{array}$ & $\begin{array}{c}0.099 \\
(0.015)\end{array}$ & $\begin{array}{c}0.143 \\
(0.001)\end{array}$ \\
\hline CGCG 247-020 & $\begin{array}{c}0.017 \\
(0.002)\end{array}$ & $\begin{array}{c}0.013 \\
(0.001)\end{array}$ & $\begin{array}{c}0.034 \\
(0.002)\end{array}$ & $\begin{array}{c}0.088 \\
(0.002)\end{array}$ & $\begin{array}{c}0.150 \\
(0.022)\end{array}$ & $\begin{array}{c}0.705 \\
(0.035)\end{array}$ & $\begin{array}{c}0.840 \\
(0.019)\end{array}$ & $\begin{array}{c}6.010 \\
(0.035)\end{array}$ & $\begin{array}{c}6.222 \\
(0.933)\end{array}$ & $\begin{array}{c}8.470 \\
(0.155)\end{array}$ & $\begin{array}{c}5.403 \\
(1.621)\end{array}$ & $\begin{array}{c}0.284 \\
(0.111)\end{array}$ & $\begin{array}{c}0.036 \\
(0.008)\end{array}$ & $\begin{array}{l}\ldots \\
(\ldots)\end{array}$ & $\begin{array}{c}0.021 \\
(0.001)\end{array}$ \\
\hline IRAS F14348-1447 & $\begin{array}{c}0.012 \\
(0.001)\end{array}$ & $\begin{array}{c}0.011 \\
(0.001)\end{array}$ & $\begin{array}{c}0.011 \\
(0.001)\end{array}$ & $\begin{array}{c}0.049 \\
(0.001)\end{array}$ & $\begin{array}{r}<0.10 \\
(\ldots)\end{array}$ & $\begin{array}{c}0.393 \\
(0.020)\end{array}$ & $\begin{array}{c}0.550 \\
(0.062)\end{array}$ & $\begin{array}{c}6.820 \\
(0.040)\end{array}$ & $\begin{array}{l}\ldots \\
(\ldots)\end{array}$ & $\begin{array}{c}7.310 \\
(0.151)\end{array}$ & $\begin{array}{l}\ldots \\
(\ldots)\end{array}$ & $\begin{array}{l}\ldots \\
(\ldots)\end{array}$ & $\begin{array}{l}\ldots \\
(\ldots)\end{array}$ & $\begin{array}{l}\ldots \\
(\ldots)\end{array}$ & $\begin{array}{c}0.037 \\
(0.001)\end{array}$ \\
\hline
\end{tabular}


Table 5

(Continued)

\begin{tabular}{|c|c|c|c|c|c|c|c|c|c|c|c|c|c|c|c|}
\hline Object & $\begin{array}{l}\text { IRAC1 } \\
3.6 \mu \mathrm{m} \\
\text { (2) }\end{array}$ & $\begin{array}{c}\text { IRAC2 } \\
4.5 \mu \mathrm{m} \\
\text { (3) }\end{array}$ & $\begin{array}{l}\text { IRAC3 } \\
5.8 \mu \mathrm{m} \\
\quad(4)\end{array}$ & $\begin{array}{c}\text { IRAC4 } \\
8.0 \mu \mathrm{m} \\
\quad(5)\end{array}$ & $\begin{array}{l}\text { IRAS1 } \\
12 \mu \mathrm{m} \\
\text { (6) }\end{array}$ & $\begin{array}{l}\text { MIPS1 } \\
24 \mu \mathrm{m} \\
\text { (7) }\end{array}$ & $\begin{array}{l}\text { IRAS2 } \\
25 \mu \mathrm{m} \\
\quad(8)\end{array}$ & $\begin{array}{l}\text { IRAS3 } \\
60 \mu \mathrm{m} \\
\text { (9) }\end{array}$ & $\begin{array}{l}\text { MIPS2 } \\
70 \mu \mathrm{m} \\
(10)\end{array}$ & $\begin{array}{l}\text { IRAS4 } \\
100 \mu \mathrm{m} \\
(11)\end{array}$ & $\begin{array}{l}\text { MIPS3 } \\
160 \mu \mathrm{m} \\
(12)\end{array}$ & $\begin{array}{c}\text { SCUBA1 } \\
450 \mu \mathrm{m} \\
(13)\end{array}$ & $\begin{array}{c}\text { SCUBA2 } \\
850 \mu \mathrm{m} \\
(14)\end{array}$ & $\begin{array}{c}\text { VLA1 } \\
4.85 \mathrm{GHz} \\
(15)\end{array}$ & $\begin{array}{c}\text { VLA2 } \\
1.49 \mathrm{GHz} \\
(16)\end{array}$ \\
\hline VV 340a & $\begin{array}{c}0.056 \\
(0.006)\end{array}$ & $\begin{array}{c}0.042 \\
(0.004)\end{array}$ & $\begin{array}{c}0.100 \\
(0.006)\end{array}$ & $\begin{array}{c}0.274 \\
(0.006)\end{array}$ & $\begin{array}{c}0.360 \\
(0.016)\end{array}$ & $\begin{array}{c}0.424 \\
(0.021)\end{array}$ & $\begin{array}{c}0.410 \\
(0.031)\end{array}$ & $\begin{array}{c}6.950 \\
(0.029)\end{array}$ & $\begin{array}{c}8.896 \\
(1.334)\end{array}$ & $\begin{array}{l}15.160 \\
(0.169)\end{array}$ & $\begin{array}{l}14.563 \\
(4.369)\end{array}$ & $(\ldots)$ & $\begin{array}{c}0.215 \\
(0.031)\end{array}$ & $\begin{array}{c}0.039 \\
(0.006)\end{array}$ & $\begin{array}{c}0.075 \\
(0.001)\end{array}$ \\
\hline CGCG 049-057 & $\begin{array}{c}0.018 \\
(0.002)\end{array}$ & $\begin{array}{c}0.013 \\
(0.001)\end{array}$ & $\begin{array}{c}0.027 \\
(0.002)\end{array}$ & $\begin{array}{c}0.056 \\
(0.002)\end{array}$ & $\begin{array}{r}<0.05 \\
(\ldots)\end{array}$ & $\begin{array}{c}0.555 \\
(0.028)\end{array}$ & $\begin{array}{c}0.950 \\
(0.029)\end{array}$ & $\begin{array}{l}21.890 \\
(0.072)\end{array}$ & $\begin{array}{c}20.487 \\
(3.073)\end{array}$ & $\begin{array}{l}31.530 \\
(0.158)\end{array}$ & $\begin{array}{l}\ldots \\
(\ldots)\end{array}$ & $\begin{array}{l}\ldots \\
(\ldots)\end{array}$ & $\begin{array}{c}0.200 \\
(0.027)\end{array}$ & $\begin{array}{l}\ldots \\
(\ldots)\end{array}$ & $\begin{array}{c}0.046 \\
(0.001)\end{array}$ \\
\hline VV 705 & $\begin{array}{c}0.028 \\
(0.003)\end{array}$ & $\begin{array}{c}0.022 \\
(0.002)\end{array}$ & $\begin{array}{c}0.065 \\
(0.003)\end{array}$ & $\begin{array}{c}0.130 \\
(0.003)\end{array}$ & $\begin{array}{c}0.290 \\
(0.019)\end{array}$ & $\begin{array}{c}1.196 \\
(0.060)\end{array}$ & $\begin{array}{c}1.420 \\
(0.016)\end{array}$ & $\begin{array}{c}9.020 \\
(0.021)\end{array}$ & $\begin{array}{c}8.479 \\
(1.272)\end{array}$ & $\begin{array}{l}10.000 \\
(0.091)\end{array}$ & $\begin{array}{c}6.308 \\
(1.892)\end{array}$ & $\begin{array}{c}0.423 \\
(0.093)\end{array}$ & $\begin{array}{c}0.060 \\
(0.014)\end{array}$ & $\begin{array}{c}0.031 \\
(0.005)\end{array}$ & $\begin{array}{c}0.048 \\
(0.001)\end{array}$ \\
\hline IRAS F15250+3608 & $\begin{array}{c}0.010 \\
(0.001)\end{array}$ & $\begin{array}{c}0.010 \\
(0.001)\end{array}$ & $\begin{array}{c}0.038 \\
(0.001)\end{array}$ & $\begin{array}{c}0.122 \\
(0.001)\end{array}$ & $\begin{array}{c}0.160 \\
(0.030)\end{array}$ & $\begin{array}{c}1.065 \\
(0.053)\end{array}$ & $\begin{array}{c}1.310 \\
(0.025)\end{array}$ & $\begin{array}{c}7.100 \\
(0.043)\end{array}$ & $\begin{array}{c}6.309 \\
(0.946)\end{array}$ & $\begin{array}{c}5.930 \\
(0.098)\end{array}$ & $\begin{array}{c}3.214 \\
(0.964)\end{array}$ & $\begin{array}{c}0.252 \\
(0.070)\end{array}$ & $\begin{array}{c}0.033 \\
(0.008)\end{array}$ & $\begin{array}{l}\ldots \\
(\ldots)\end{array}$ & $\begin{array}{c}0.014 \\
(0.001)\end{array}$ \\
\hline UGC 09913 & $\begin{array}{c}0.054 \\
(0.005)\end{array}$ & $\begin{array}{c}0.045 \\
(0.005)\end{array}$ & $\begin{array}{c}0.137 \\
(0.005)\end{array}$ & $\begin{array}{c}0.251 \\
(0.005)\end{array}$ & $\begin{array}{c}0.610 \\
(0.021)\end{array}$ & $\begin{array}{c}4.010 \\
(0.201)\end{array}$ & $\begin{array}{l}8.000 \\
(0.034)\end{array}$ & $\begin{array}{r}104.090 \\
(0.112)\end{array}$ & $\begin{array}{c}80.771 \\
(12.116)\end{array}$ & $\begin{array}{r}115.290 \\
(0.138)\end{array}$ & $\begin{array}{l}\ldots \\
(\ldots)\end{array}$ & $\begin{array}{l}\ldots \\
(\ldots)\end{array}$ & $\begin{array}{c}0.832 \\
(0.086)\end{array}$ & $\begin{array}{c}0.208 \\
(0.031)\end{array}$ & $\begin{array}{c}0.324 \\
(0.001)\end{array}$ \\
\hline NGC 6090 & $\begin{array}{c}0.033 \\
(0.003)\end{array}$ & $\begin{array}{c}0.023 \\
(0.002)\end{array}$ & $\begin{array}{c}0.061 \\
(0.003)\end{array}$ & $\begin{array}{c}0.177 \\
(0.003)\end{array}$ & $\begin{array}{c}0.260 \\
(0.020)\end{array}$ & $\begin{array}{c}0.954 \\
(0.048)\end{array}$ & $\begin{array}{c}1.240 \\
(0.021)\end{array}$ & $\begin{array}{c}6.480 \\
(0.030)\end{array}$ & $\begin{array}{c}6.762 \\
(1.014)\end{array}$ & $\begin{array}{c}9.410 \\
(0.102)\end{array}$ & $\begin{array}{c}7.233 \\
(2.170)\end{array}$ & $\begin{array}{l}\ldots \\
(\ldots)\end{array}$ & $\begin{array}{l}\ldots \\
(\ldots)\end{array}$ & $\begin{array}{l}\ldots \\
(\ldots)\end{array}$ & $\begin{array}{c}0.046 \\
(0.001)\end{array}$ \\
\hline CGCG 052-037 & $\begin{array}{c}0.041 \\
(0.004)\end{array}$ & $\begin{array}{c}0.030 \\
(0.003)\end{array}$ & $\begin{array}{c}0.082 \\
(0.004)\end{array}$ & $\begin{array}{c}0.222 \\
(0.004)\end{array}$ & $\begin{array}{c}0.250 \\
(0.030)\end{array}$ & $\begin{array}{c}0.786 \\
(0.039)\end{array}$ & $\begin{array}{c}0.810 \\
(0.031)\end{array}$ & $\begin{array}{l}7.000 \\
(0.022)\end{array}$ & $\begin{array}{c}7.559 \\
(1.134)\end{array}$ & $\begin{array}{l}11.230 \\
(0.133)\end{array}$ & $\begin{array}{c}9.288 \\
(2.786)\end{array}$ & $\begin{array}{l}\ldots \\
(\ldots)\end{array}$ & $\begin{array}{c}0.095 \\
(0.018)\end{array}$ & $\begin{array}{l}\ldots \\
(\ldots)\end{array}$ & $\begin{array}{c}0.031 \\
(0.001)\end{array}$ \\
\hline NGC 6286 & $\begin{array}{c}0.065 \\
(0.007)\end{array}$ & $\begin{array}{c}0.047 \\
(0.005)\end{array}$ & $\begin{array}{c}0.134 \\
(0.007)\end{array}$ & $\begin{array}{c}0.329 \\
(0.007)\end{array}$ & $\begin{array}{c}0.470 \\
(0.015)\end{array}$ & $\begin{array}{c}0.581 \\
(0.029)\end{array}$ & $\begin{array}{c}0.620 \\
(0.017)\end{array}$ & $\begin{array}{c}9.240 \\
(0.043)\end{array}$ & $\begin{array}{l}13.129 \\
(1.969)\end{array}$ & $\begin{array}{l}23.110 \\
(0.048)\end{array}$ & $\begin{array}{l}21.270 \\
(6.381)\end{array}$ & $\begin{array}{l}\ldots \\
(\ldots)\end{array}$ & $\begin{array}{l}\ldots \\
(\ldots)\end{array}$ & $\begin{array}{l}\ldots \\
(\ldots)\end{array}$ & $\begin{array}{c}0.156 \\
(0.001)\end{array}$ \\
\hline IRAS F17132+5313 & $\begin{array}{c}0.017 \\
(0.002)\end{array}$ & $\begin{array}{c}0.014 \\
(0.001)\end{array}$ & $\begin{array}{c}0.030 \\
(0.002)\end{array}$ & $\begin{array}{c}0.105 \\
(0.002)\end{array}$ & $\begin{array}{c}0.240 \\
(0.021)\end{array}$ & $\begin{array}{c}0.493 \\
(0.025)\end{array}$ & $\begin{array}{c}0.650 \\
(0.024)\end{array}$ & $\begin{array}{c}6.070 \\
(0.034)\end{array}$ & $\begin{array}{c}6.115 \\
(0.917)\end{array}$ & $\begin{array}{c}7.900 \\
(0.105)\end{array}$ & $\begin{array}{c}5.576 \\
(1.673)\end{array}$ & $\begin{array}{l}\ldots \\
(\ldots)\end{array}$ & $\begin{array}{l}\ldots \\
(\ldots)\end{array}$ & $\begin{array}{l}\ldots \\
(\ldots)\end{array}$ & $\begin{array}{c}0.026 \\
(0.001)\end{array}$ \\
\hline ESO 602-G025 & $\begin{array}{c}0.043 \\
(0.004)\end{array}$ & $\begin{array}{c}0.037 \\
(0.004)\end{array}$ & $\begin{array}{c}0.098 \\
(0.004)\end{array}$ & $\begin{array}{c}0.229 \\
(0.004)\end{array}$ & $\begin{array}{c}0.270 \\
(0.029)\end{array}$ & $\begin{array}{c}0.608 \\
(0.030)\end{array}$ & $\begin{array}{c}0.910 \\
(0.045)\end{array}$ & $\begin{array}{l}5.420 \\
(0.044)\end{array}$ & $\begin{array}{c}6.359 \\
(0.954)\end{array}$ & $\begin{array}{c}9.640 \\
(0.082)\end{array}$ & $\begin{array}{c}7.844 \\
(2.353)\end{array}$ & $\begin{array}{l}\ldots \\
(\ldots)\end{array}$ & $\begin{array}{l}\ldots \\
(\ldots)\end{array}$ & $\begin{array}{l}\ldots \\
(\ldots)\end{array}$ & $\begin{array}{c}0.041 \\
(0.001)\end{array}$ \\
\hline IRAS F22491-1808 & $\begin{array}{c}0.009 \\
(0.001)\end{array}$ & $\begin{array}{c}0.004 \\
(0.000)\end{array}$ & $\begin{array}{c}0.003 \\
(0.001)\end{array}$ & $\begin{array}{c}0.028 \\
(0.001)\end{array}$ & $\begin{array}{r}<0.09 \\
(\ldots)\end{array}$ & $\begin{array}{c}0.433 \\
(0.022)\end{array}$ & $\begin{array}{c}0.540 \\
(0.067)\end{array}$ & $\begin{array}{c}5.540 \\
(0.036)\end{array}$ & $\begin{array}{c}4.744 \\
(0.712)\end{array}$ & $\begin{array}{c}4.640 \\
(0.095)\end{array}$ & $\begin{array}{c}3.014 \\
(0.904)\end{array}$ & $\begin{array}{l}\ldots \\
(\ldots)\end{array}$ & $\begin{array}{l}\ldots \\
(\ldots)\end{array}$ & $\begin{array}{l}\ldots \\
(\ldots)\end{array}$ & $\begin{array}{c}0.006 \\
(0.001)\end{array}$ \\
\hline NGC 7469 & $\begin{array}{c}0.209 \\
(0.021)\end{array}$ & $\begin{array}{c}0.187 \\
(0.019)\end{array}$ & $\begin{array}{c}0.665 \\
(0.021)\end{array}$ & $\begin{array}{c}0.771 \\
(0.021)\end{array}$ & $\begin{array}{c}1.590 \\
(0.039)\end{array}$ & $\begin{array}{c}4.080 \\
(0.204)\end{array}$ & $\begin{array}{c}5.960 \\
(0.032)\end{array}$ & $\begin{array}{c}27.330 \\
(0.040)\end{array}$ & $\begin{array}{l}25.034 \\
(3.755)\end{array}$ & $\begin{array}{l}35.160 \\
(0.599)\end{array}$ & $\begin{array}{l}\ldots \\
(\ldots)\end{array}$ & $\begin{array}{l}\ldots \\
(\ldots)\end{array}$ & $\begin{array}{c}0.264 \\
(0.030)\end{array}$ & $\begin{array}{c}0.071 \\
(0.011)\end{array}$ & $\begin{array}{c}0.169 \\
(0.001)\end{array}$ \\
\hline CGCG 453-062 & $\begin{array}{c}0.032 \\
(0.003)\end{array}$ & $\begin{array}{c}0.022 \\
(0.002)\end{array}$ & $\begin{array}{c}0.056 \\
(0.003)\end{array}$ & $\begin{array}{c}0.136 \\
(0.003)\end{array}$ & $\begin{array}{c}0.190 \\
(0.038)\end{array}$ & $\begin{array}{c}0.411 \\
(0.021)\end{array}$ & $\begin{array}{c}0.540 \\
(0.038)\end{array}$ & $\begin{array}{c}7.190 \\
(0.047)\end{array}$ & $\begin{array}{c}7.619 \\
(1.143)\end{array}$ & $\begin{array}{l}11.730 \\
(0.163)\end{array}$ & $\begin{array}{l}10.317 \\
(3.095)\end{array}$ & $\begin{array}{l}\ldots \\
(\ldots)\end{array}$ & $\begin{array}{c}0.069 \\
(0.014)\end{array}$ & $\begin{array}{l}\ldots \\
(\ldots)\end{array}$ & $\begin{array}{c}0.037 \\
(0.001)\end{array}$ \\
\hline IC 5298 & $\begin{array}{c}0.037 \\
(0.004)\end{array}$ & $\begin{array}{c}0.045 \\
(0.005)\end{array}$ & $\begin{array}{c}0.048 \\
(0.004)\end{array}$ & $\begin{array}{c}0.132 \\
(0.004)\end{array}$ & $\begin{array}{c}0.340 \\
(0.024)\end{array}$ & $\begin{array}{c}1.427 \\
(0.071)\end{array}$ & $\begin{array}{c}1.950 \\
(0.053)\end{array}$ & $\begin{array}{c}9.060 \\
(0.051)\end{array}$ & $\begin{array}{c}8.424 \\
(1.264)\end{array}$ & $\begin{array}{l}11.990 \\
(0.097)\end{array}$ & $\begin{array}{c}6.774 \\
(2.032)\end{array}$ & $\begin{array}{l}\ldots \\
(\ldots)\end{array}$ & $\begin{array}{c}0.077 \\
(0.015)\end{array}$ & $\begin{array}{l}\ldots \\
(\ldots)\end{array}$ & $\begin{array}{c}0.034 \\
(0.001)\end{array}$ \\
\hline NGC 7592 & $\begin{array}{c}0.044 \\
(0.004)\end{array}$ & $\begin{array}{c}0.037 \\
(0.004)\end{array}$ & $\begin{array}{c}0.091 \\
(0.004)\end{array}$ & $\begin{array}{c}0.214 \\
(0.004)\end{array}$ & $\begin{array}{c}0.260 \\
(0.031)\end{array}$ & $\begin{array}{c}0.868 \\
(0.043)\end{array}$ & $\begin{array}{c}0.970 \\
(0.056)\end{array}$ & $\begin{array}{c}8.050 \\
(0.049)\end{array}$ & $\begin{array}{c}7.445 \\
(1.117)\end{array}$ & $\begin{array}{l}10.580 \\
(0.137)\end{array}$ & $\begin{array}{c}8.833 \\
(2.650)\end{array}$ & $\begin{array}{l}\ldots \\
(\ldots)\end{array}$ & $\begin{array}{c}0.108 \\
(0.019)\end{array}$ & $\begin{array}{l}\ldots \\
(\ldots)\end{array}$ & $\begin{array}{c}0.062 \\
(0.001)\end{array}$ \\
\hline NGC 7674 & $\begin{array}{c}0.090 \\
(0.009)\end{array}$ & $\begin{array}{c}0.093 \\
(0.009)\end{array}$ & $\begin{array}{c}0.202 \\
(0.009)\end{array}$ & $\begin{array}{c}0.295 \\
(0.009)\end{array}$ & $\begin{array}{c}0.680 \\
(0.037)\end{array}$ & $\begin{array}{c}1.600 \\
(0.080)\end{array}$ & $\begin{array}{c}1.920 \\
(0.034)\end{array}$ & $\begin{array}{c}5.360 \\
(0.042)\end{array}$ & $\begin{array}{c}5.881 \\
(0.882)\end{array}$ & $\begin{array}{c}8.330 \\
(0.141)\end{array}$ & $\begin{array}{c}7.966 \\
(2.390)\end{array}$ & $\begin{array}{l}\ldots \\
(\ldots)\end{array}$ & $\begin{array}{c}0.108 \\
(0.020)\end{array}$ & $\begin{array}{c}0.086 \\
(0.013)\end{array}$ & $\begin{array}{c}0.209 \\
(0.001)\end{array}$ \\
\hline NGC 7770/1 & $\begin{array}{c}0.321 \\
(0.032)\end{array}$ & $\begin{array}{c}0.213 \\
(0.021)\end{array}$ & $\begin{array}{c}0.447 \\
(0.032)\end{array}$ & $\begin{array}{c}0.958 \\
(0.032)\end{array}$ & $\begin{array}{c}0.990 \\
(0.036)\end{array}$ & $\begin{array}{c}1.773 \\
(0.089)\end{array}$ & $\begin{array}{c}2.170 \\
(0.036)\end{array}$ & $\begin{array}{l}19.670 \\
(0.135)\end{array}$ & $\begin{array}{c}23.341 \\
(3.501)\end{array}$ & $\begin{array}{l}40.120 \\
(0.839)\end{array}$ & $\begin{array}{l}\ldots \\
(\ldots)\end{array}$ & $\begin{array}{l}\ldots \\
(\ldots)\end{array}$ & $\begin{array}{c}0.377 \\
(0.042)\end{array}$ & $\begin{array}{l}\ldots \\
(\ldots)\end{array}$ & $\begin{array}{c}0.124 \\
(0.001)\end{array}$ \\
\hline MRK 0331 & $\begin{array}{c}0.054 \\
(0.005)\end{array}$ & $\begin{array}{c}0.039 \\
(0.004)\end{array}$ & $\begin{array}{c}0.133 \\
(0.005)\end{array}$ & $\begin{array}{c}0.320 \\
(0.005)\end{array}$ & $\begin{array}{c}0.520 \\
(0.035)\end{array}$ & $\begin{array}{l}\ldots \\
(\ldots)\end{array}$ & $\begin{array}{c}2.540 \\
(0.026)\end{array}$ & $\begin{array}{l}18.000 \\
(0.046)\end{array}$ & $\begin{array}{l}\ldots \\
(\ldots)\end{array}$ & $\begin{array}{l}22.700 \\
(0.194)\end{array}$ & $\begin{array}{l}\ldots \\
(\ldots)\end{array}$ & $\begin{array}{l}\ldots \\
(\ldots)\end{array}$ & $\begin{array}{c}0.132 \\
(0.025)\end{array}$ & $\begin{array}{c}0.028 \\
(0.006)\end{array}$ & $\begin{array}{c}0.068 \\
(0.001)\end{array}$ \\
\hline
\end{tabular}

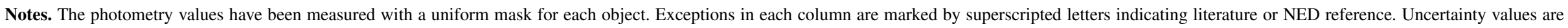
indicated in parantheses where available.

Columns 2-5: Spitzer-IRAC photometry from images in J. M. Mazzarella et al. (2012, in preparation) and J. A. Surace (2012, in preparation).

Column 6: IRAS photometry from Sanders et al. (2003).

Column 7: Spitzer-MIPS24 photometry from images in J. M. Mazzarella et al. (2012, in preparation) and J. A. Surace (2012, in preparation).

Columns 8 and 9: IRAS photometry from Sanders et al. (2003).

Column 10: Spitzer-MIPS70 photometry from images in J. M. Mazzarella et al. (2012, in preparation) and J. A. Surace (2012, in preparation).

Column 11: IRAS photometry from Sanders et al. (2003).

Column 12: Spitzer-MIPS160 photometry from images in J. M. Mazzarella et al. (2012, in preparation) and J. A. Surace (2012, in preparation).

Columns 13 and 14: JCMT/SCUBA submillimeter photometry from Dunne et al. (2000) and Dunne \& Eales (2001).

Columns 15 and 16: VLA photometry from Condon et al. $(1990,1991)$.

${ }^{a}$ At $1.425 \mathrm{GHz}$; photometry from Condon et al. (1996). 

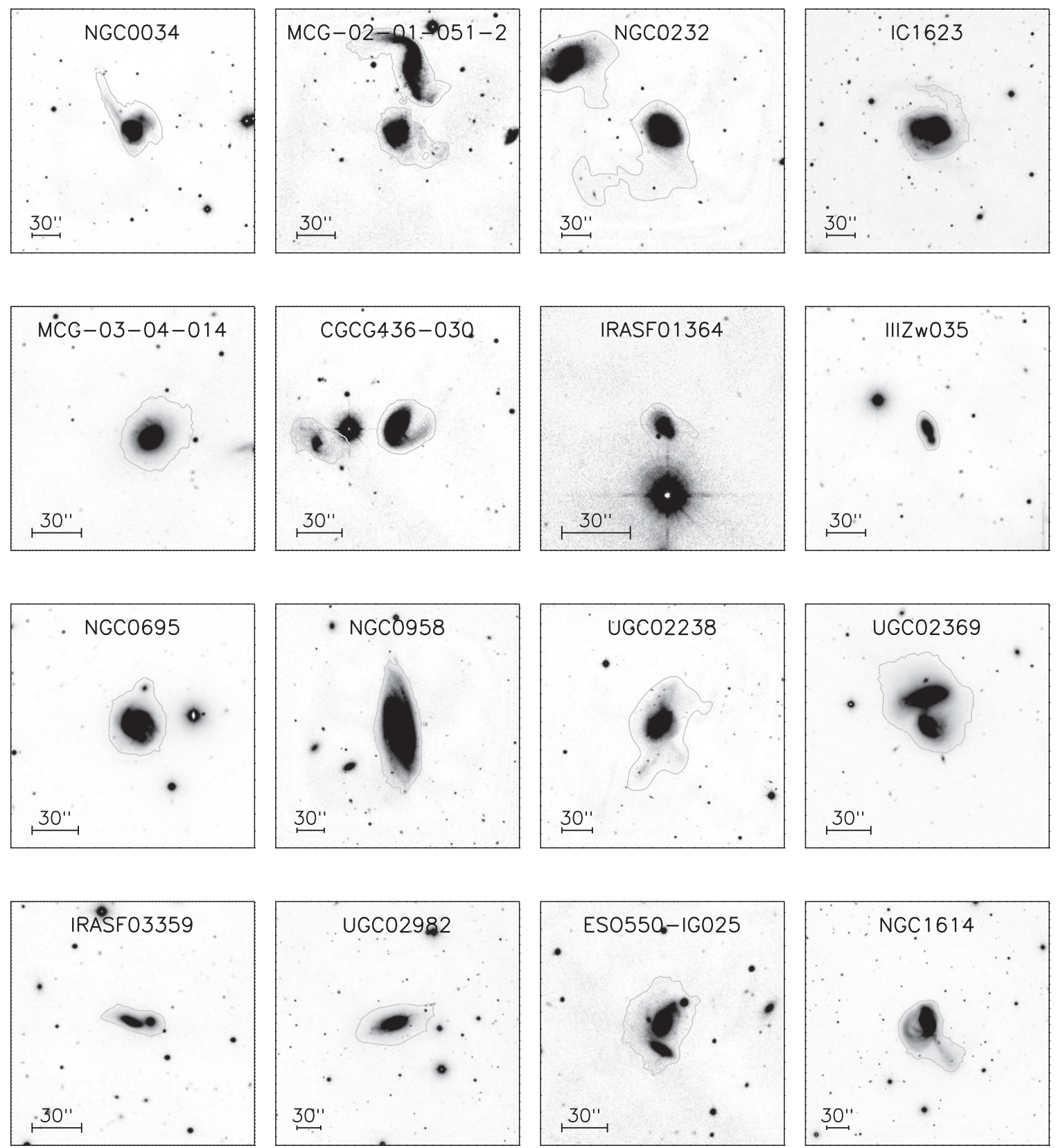

Figure 1. HST and UH $2.2 \mathrm{~m}$ I-band images in increasing R.A. order for the 64 local (U)LIRGs with mask photometry contour superimposed. The field of view for all images is $100 \mathrm{kpc} \times 100 \mathrm{kpc}$, and a $30^{\prime \prime}$ scale bar is drawn inside each frame to help guide the eye.

separately. Qualitatively speaking, the overall SED shape is similar for all of the LIRGs and ULIRGs, with two significant maxima, one of which is near $1 \mu \mathrm{m}$ and the other near $80 \mu \mathrm{m}$, with a large dip between them, and all SEDs falling toward the FUV and at submillimeter wavelengths. The logarithmic difference between the stellar and dust peaks is 1.2 dex for the ULIRGs but only $0.7 \mathrm{dex}$ for the LIRGs, providing a quantitative measure for the change in stellar-to-dust ratio with infrared luminosity. The next two sections offer different ways to quantify the spectral shapes. The fit data values for the mean SEDs for both LIRGs and ULIRGs are given in Table 6.

\subsection{Spectral Indices}

A useful and more quantitative way of discussing the spectral shapes of SEDs makes use of spectral indices defined as follows:

$$
\alpha_{2}^{1}=\frac{\log \nu_{1} f_{\nu_{1}}-\log v_{2} f_{\nu_{2}}}{\log v_{1}-\log \nu_{2}}
$$

where the indices ( 1 and 2 ) correspond to observed wavelengths in $\mu \mathrm{m}$. To mirror a high- $z$ SED study of a $70 \mu \mathrm{m}$ selected sample of (U)LIRGs (Kartaltepe et al. 2010), here we have chosen three wavelength ranges where the SEDs appear to show the largest variations-in the UV-optical $(0.23-0.54 \mu \mathrm{m})$, NIR $(2.2-4.5 \mu \mathrm{m})$, and the MIR $(8-24 \mu \mathrm{m})$. These three wavelength ranges correspond to the shaded regions shown in Figure 4. The top panels in Figure 5 show $\alpha_{2}^{1}$ versus $L_{\mathrm{IR}}$ for three different spectral indices, corresponding to the three wavelength ranges described above. The mean values of $\alpha_{2}^{1}$ for LIRGs and ULIRGs along with a regression analysis for the full subsample of 64 objects is given in Table 7 . 

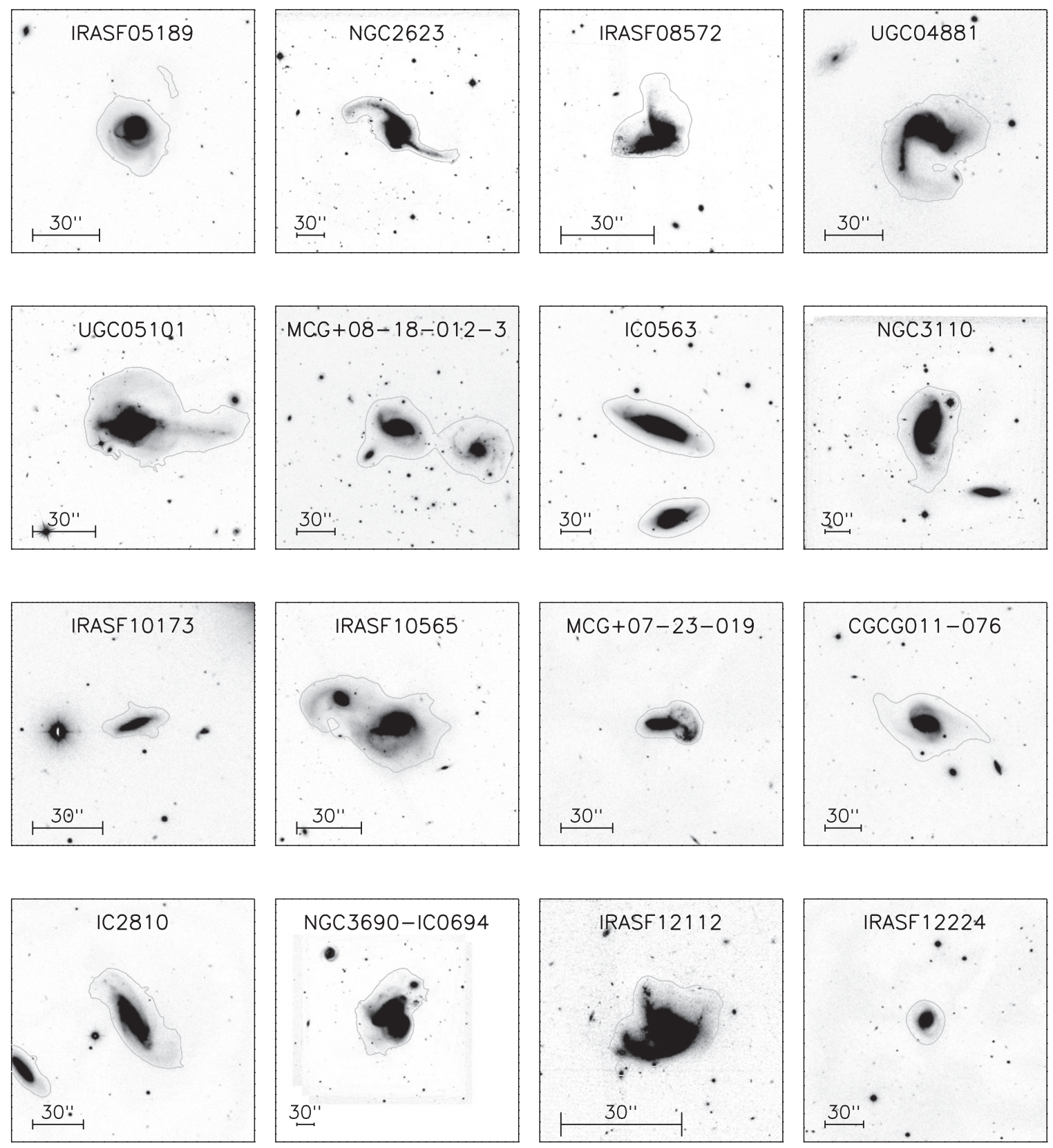

Figure 1. (Continued)

In Figure 6, we compare spectral indices: $\alpha_{0.23}^{0.54}$ versus $\alpha_{2.2}^{4.5}$ contrasts the slopes on either side of the stellar "bump;" $\alpha_{0.23}^{0.54}$ versus $\alpha_{8}^{24}$ contrasts the blueward slopes of the optical and the infrared "bumps," respectively; $\alpha_{2.2}^{4.5}$ versus $\alpha_{8}^{24}$ contrasts the redward and blueward slopes of the optical and infrared "bumps," respectively. The correlation coefficients for these three sets of comparisons are $-0.08,-0.03$, and -0.01 . With this sample size, the conservative, non-directional $p$-values are $0.57,0.83$, and 0.94 , respectively. This indicates that there is no correlation found between each pair of spectral indices. However, we note that for all three spectral index comparisons, the ULIRGs tend to show smaller values of $\alpha_{8}^{24}$ and $\alpha_{2.2}^{4.5}$, corresponding to a deeper trough at $\lambda \sim 4-8 \mu \mathrm{m}$, presumably due to greater silicate dust absorption of the continuum in ULIRGs. The physical significance of using $\alpha_{2.2}^{4.5}$ as an AGN indicator is further discussed in Section 5.1.5.

\subsection{Flux Ratios}

The SED shapes can also be characterized in terms of flux ratios with respect to the measured flux at $60 \mu \mathrm{m}$ to compare direct stellar emission to dust emission. Figure 7 shows the distribution of flux ratios at radio $(1.4 \mathrm{GHz}), J$ band $(1.2 \mu \mathrm{m})$, NUV $(0.23 \mu \mathrm{m})$, and HX (2-10 keV), for all 11 ULIRGs and 53 LIRGs, where data are available. The wavelengths chosen for display represent the short and long wavelength extremes of the SEDs as well as the "peak" and the short wavelength side of the stellar thermal bump. For the $J$-band and NUV ratios, the difference in the distributions between ULIRGs and LIRGs is simply due to the well-known property of the SEDs where the thermal stellar "bump" remains relatively constant $(\lesssim 2)$ while the thermal dust "bump" grows by a factor of $\sim 10$ (e.g., Sanders \& Mirabel 1996). The different distributions for the LIRGs and ULIRGs point to the discrepant starlight-to-dust ratios in the 

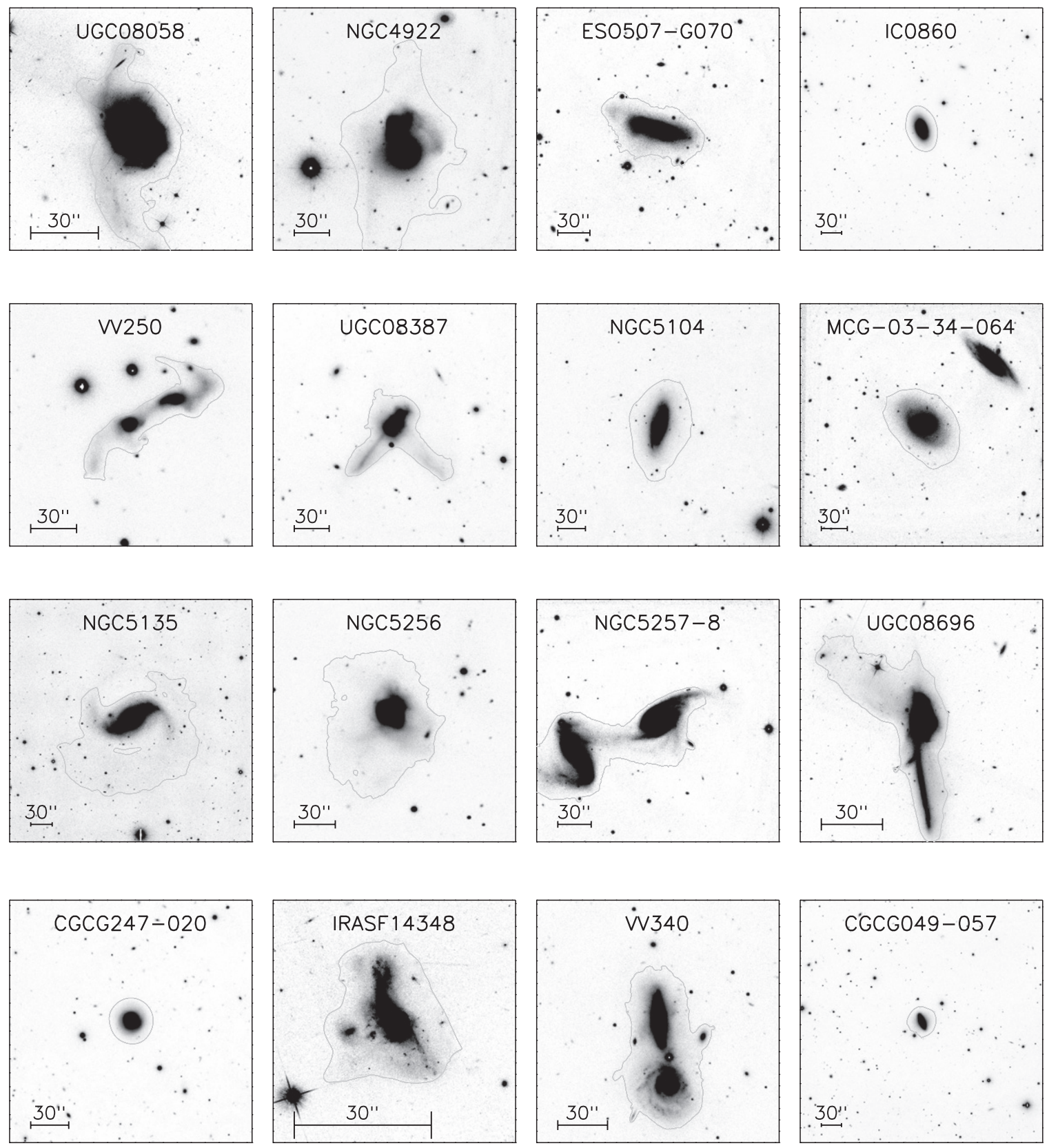

Figure 1. (Continued)

two populations. The HX band shows a somewhat surprising result in that the ratio seems to be similar for both LIRGs and ULIRGs.

Finally, we summarize the SEDs of our complete sample of (U)LIRGs in Table 8, which lists the relative mean luminosity, $R=\log \left[L_{v}\right.$ (band) $\left./ L_{v}(60 \mu \mathrm{m})\right]$, in all 26 observed bands with respect to the mean luminosity at $60 \mu \mathrm{m}$. Table 8 also lists the dispersion in the luminosity ratio, $\sigma_{R}$, and the full range, $\Delta R$. The largest values of $R$ are found at the long and short wavelength ends of the SEDs, where the emission is clearly not fit by the two (stellar and dust) thermal "bumps." However, it is interesting to note that a small dispersion in $R$ is found in the radio $(1.4 \mathrm{GHz})$, where the ratio is largest $(R=-5.81)$. This would seem to confirm that the well-known "radio-infrared correlation" (e.g., Helou et al. 1985), also holds for (U)LIRGs.

At X-ray wavelengths, there seems to be a 0.4 dex increase in the mean luminosity in the HX band compared with the SX band. Of the 26 objects within our sample that are detected in both X-ray bands, $\sim 58 \%$ are more luminous in HX than in SX, suggesting the presence of an HX ionizing source. Due to the relative incompleteness of X-ray observations at the lower-luminosity end of our sample, this is slightly higher than the conservative estimate of $37 \%$ AGN fraction (or $48 \%$ if $[\mathrm{Ne} \mathrm{V}]$ detection is taken into account) from an X-ray study of 44 GOALS (U)LIRGs at the high end of the $L_{\mathrm{IR}}$ range (with median $\log \left(L_{\mathrm{IR}} / L_{\odot}\right)=11.99$; Iwasawa et al. 2011). Incorporating the entire GOALS sample, Petric et al. (2011) found that $18 \%$ of all (U)LIRGs contain an AGN based on a mid-IR Spitzer-IRS study; the comparatively lower fraction reflects that fewer of the lower-luminosity objects feature an AGN. We note that because of the complexity of dust geometry within these systems, discrepancies among the AGN fractions thus determined may be due to the limited sensitivity of the various AGN indicators. 

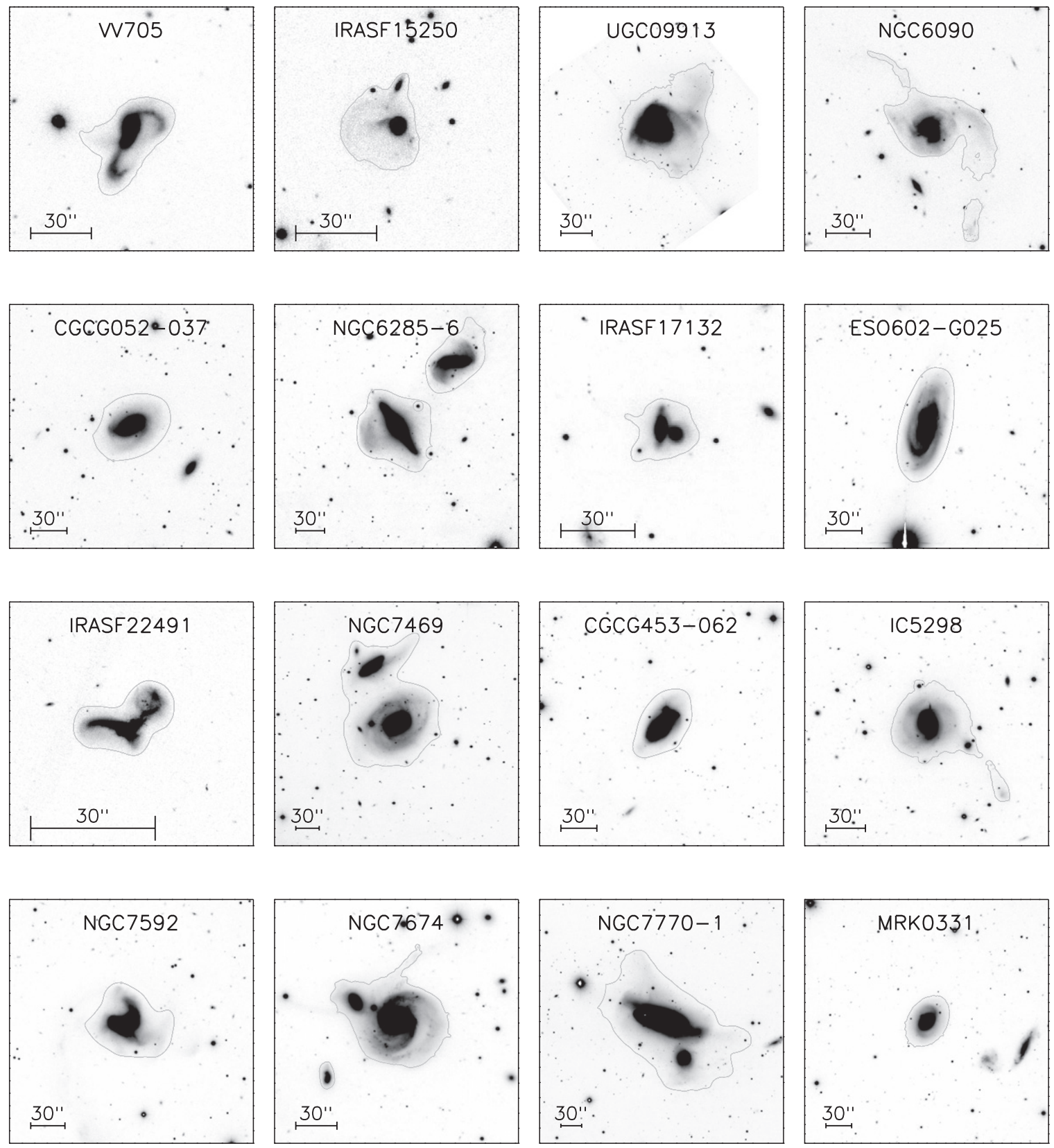

Figure 1. (Continued)

\section{PHYSICAL PROPERTIES OF THE GOALS SAMPLE}

Here we discuss the template fitting done to compute infrared luminosity and stellar mass; specifically, we fit the MIR-submillimeter portion of the SED with various dust models to compute $L[8-1000 \mu \mathrm{m}]$ and compare with previous estimates of $L_{\mathrm{IR}}$ computed from IRAS photometry. We use population synthesis models to fit the UV-NIR portion of the SED in order to determine stellar mass. These masses are then compared with stellar mass estimates computed using $H$-band luminosities alone.

\subsection{Template Fits}

We have fitted each of the (U)LIRG SEDs with stellar population synthesis and dust models (see Figure 2). Our goals are twofold: (1) to better determine stellar mass $\left(M_{\star}\right)$ and subsequently star formation rate (SFR) of the local (U)LIRGs, and (2) to better estimate the flux at any unobserved wavelength band. The optical through NIR SED fitting has been done using the Le PHotometric Analysis for Redshift Estimations (Le PHARE ${ }^{18}$ ) code, a photometric redshift and simulation package developed by S. Arnouts and O. Ilbert. It is capable of providing optical and FIR fitting as well as a complete treatment of physical parameters and uncertainties based on the simple stellar population synthesis model of choice. As described below we consider both a Salpeter (Salpeter 1955) and Chabrier (Chabrier 2003) initial mass function (IMF) for all of our sources, plus a Calzetti extinction law (Calzetti et al. 1994) adopted throughout. Stellar masses were determined from fitting the observed data shortward of the $K$ band.

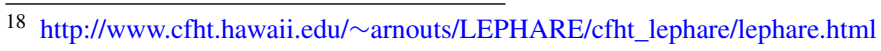






Figure 2. SEDs of the 64 objects in our sample in R.A. order. The units are $\log v(\mathrm{~Hz})$ on the $x$-axis and $\log v L_{v}\left(L_{\odot}\right)$ on the $y$-axis. The crosses represent our photometry data points, while the dashed line illustrates the UV-NIR fit to Bruzual \& Charlot (2003) stellar population synthesis models. The mBB graybody fit composes of the mid-infrared power-law portion (dotted) and the far-infrared blackbody portion (solid) in the SED.

\subsubsection{Infrared Luminosities, Dust Temperatures, and Dust Masses}

The infrared luminosity, $L_{\mathrm{IR}}$, that is discussed throughout this paper refers to the luminosity emitted in the wavelength range $8-1000 \mu \mathrm{m}$. The values of $L_{\mathrm{IR}}$ given in Table 1 have been adopted from the RBGS using the following prescription reproduced from Perault (1987) and Sanders \& Mirabel (1996):

$$
\begin{gathered}
F_{\mathrm{IR}}=1.8 \times 10^{-14}\left(13.45 f_{12}+5.16 f_{25}\right. \\
\left.+2.58 f_{60}+f_{100}\right)\left[\mathrm{Wm}^{-2}\right], \\
L[8-1000 \mu \mathrm{m}]=4 \pi D_{L}^{2} F_{\mathrm{IR}}\left[L_{\odot}\right],
\end{gathered}
$$

where $f_{12}, f_{25}, f_{60}$, and $f_{100}$ are the flux densities in Jy at 12,25 , 60 , and $100 \mu \mathrm{m}$, respectively, and $D_{L}$ is the luminosity distance.

Given the availability of our new IRAC, MIPS, and SCUBA data points, we can now test the above IRAS approximation and the validity of the assumed SED, in particular longward of $100 \mu \mathrm{m}$, for the local (U)LIRG population. We compute a new total infrared luminosity, $L_{\mathrm{IR}}=8-1000 \mu \mathrm{m}$, by using $\chi^{2}$ minimization to fit the MIR-FIR-submillimeter portion (14 data points) of the SEDs with different dust models and a modified blackbody fit (CE, DH, SK, mBB; Chary \& Elbaz 2001; Dale \& Helou 2002; Siebenmorgen \& Krügel 2007; Casey 2012; Table 9). For the CE models, 105 templates with infrared luminosity ranging from $L_{\mathrm{IR}}=10^{8.44}$ to $L_{\mathrm{IR}}=10^{13.55}$ have been used. For the DH models, the 64 templates employed exhibit infrared luminosity within the range of $10^{8.32}-10^{14.34}$. The SK template library in LEPHARE consists of spatially integrated SEDs computed for starbursts of different radii, total luminosity, visual extinction, dust density within hot spots, and the luminosity ratio of hot spots to total as a secondary parameter based on Siebenmorgen \& Krügel (2007). We utilized the models with radii $=1 \mathrm{kpc}, L_{\mathrm{IR}}=10^{10.1}-10^{14.7}, A_{V}=$ 2.2-119 mag, $n_{h s}=10^{2}-10^{4} \mathrm{~cm}^{-3}$, and OB luminosity to total 


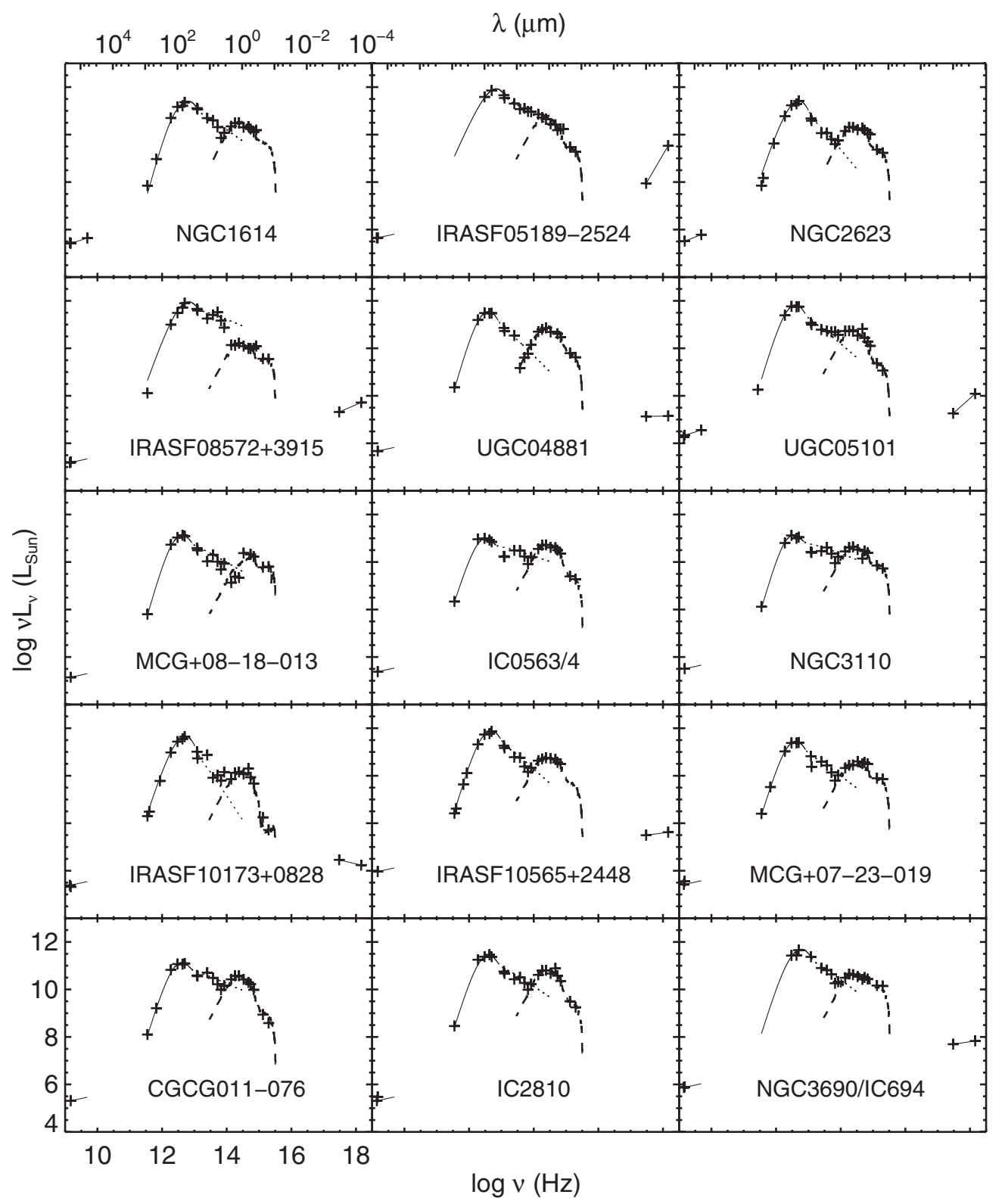

Figure 2. (Continued)

luminosity of $40 \%-90 \%$. Lastly, the model-independent mBB fit is essentially the sum of a mid-infrared power law at $\lambda<50 \mu \mathrm{m}$ and a single-temperature graybody at $\lambda>50 \mu \mathrm{m}$ (Casey 2012):

$$
S(\lambda)=N_{b b} \frac{\left(1-e^{-\left(\frac{\lambda_{0}}{\lambda}\right)^{\beta}}\right)\left(\frac{c}{\lambda}\right)^{3}}{e^{h c / \lambda k T}-1}+N_{p l} \lambda^{\alpha} e^{-\left(\frac{\lambda}{\lambda_{c}}\right)^{2}},
$$

where $T$ is the far infrared dust temperature, $\lambda_{0}$ is the wavelength corresponding to an optical depth of unity, $\lambda_{c}$ is the wavelength, where the mid-infrared power law turns over, $\beta$ is the emissivity, and $\alpha$ is the spectral index of the power-law component. We let $\beta$ and $\alpha$ vary to fit the SED where adequate data exist (see more details in Casey 2012).

The comparison of $L_{\mathrm{IR}}$ values is shown in Figure 8 . The CE and DH model fits have large scatters $(0.07$ dex and 0.08 dex $)$ around the IRAS values. The SK model fits exhibit a large scatter $(0.05 \mathrm{dex})$ around the systematic offset of $-0.03 \mathrm{dex}$ from the IRAS luminosities. The mBB fit values are $\sim 0.02$ dex systematically lower than the IRAS values, which translates to $\sim 5 \%$ of the infrared luminosity at $L_{\mathrm{IR}}=10^{12} L_{\odot}$ level. The disagreement with the IRAS values is primarily due to the inadequate color assumptions implied in the coefficients of Equation (2). The scatter and discrepancy exhibited by the luminosities derived from the model-dependent templates are due to limitations in the step size within the model grids.

Comparing the fits of the different templates and the $\mathrm{mBB}$ at $12,25,60,100$, and $850 \mu \mathrm{m}$, the residuals between the data and the different fits are the smallest for $\mathrm{mBB}$. The SK fits result in similarly minimal residuals except at the short wavelengths, whereas the $\mathrm{CE}$ and $\mathrm{DH}$ fit residuals exhibit the largest scatters. The main reason for the mismatch to the data is that the detailed infrared SED models have many degrees of freedom and very large template libraries at discrete temperatures and other grid parameters. In contrast, the analytical $\mathrm{mBB}$ fit provides more fitting flexibility and is designed to represent the data as 


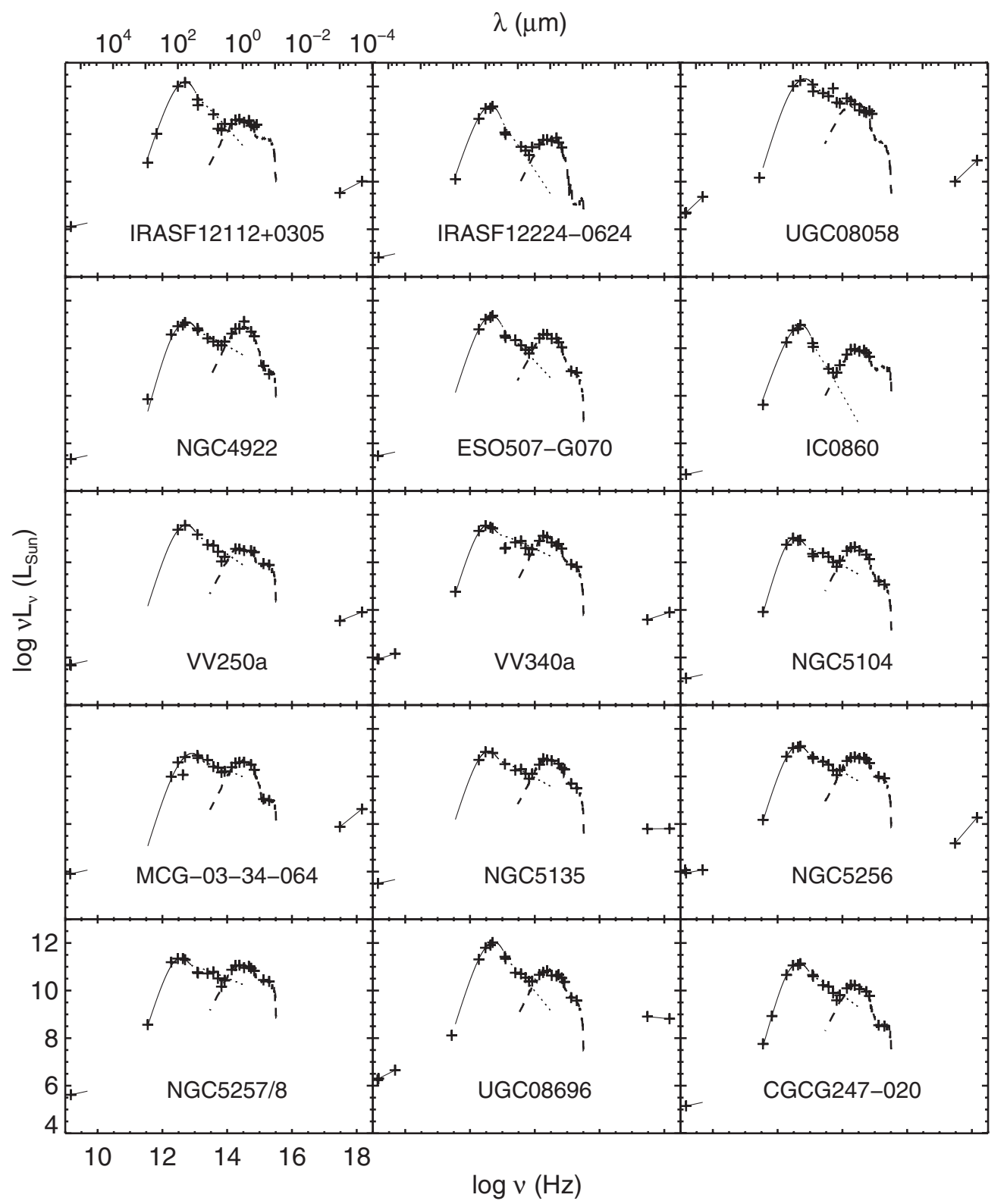

Figure 2. (Continued)

accurately as possible, despite having only three free parameters and not accounting for the mid-infrared polycyclic aromatic hydrocarbon features. Since for this paper we only measure $L_{\mathrm{IR}}$, $T_{\text {dust }}$, and $M_{\text {dust }}$, we choose to adopt the mBB fit results for the rest of the analyses.

Fitting graybody of a single dust temperature to the FIR SED from the blackbody peak $(\sim 100 \mu \mathrm{m})$ to $1000 \mu \mathrm{m}$, we determine and present the temperature of the dust in Table 9: mean $T_{\text {dust }}=33.2 \pm 6.2 \mathrm{~K}$. This temperature is $\sim 10 \mathrm{~K}$ cooler than that determined from the Perault (1987) prescription (see discussion in Casey 2012). With the lack of long-wavelength data points, the latter assumes a single-temperature dust emissivity model $\epsilon \propto v^{-1}$ fit to the fluxes in the four IRAS bands (as described in Sanders \& Mirabel 1996), often adopting a peak shortward of the true peak now revealed when data points longward of $100 \mu \mathrm{m}$ are present. The MIPS $160 \mu \mathrm{m}$ and the SCUBA $850 \mu \mathrm{m}$ points are invaluable for constraining the real peak, and this will be nailed when the Herschel far-infrared imaging observations are completed for the entire GOALS sample. Dust masses were calculated using the $850 \mu \mathrm{m}$ flux from the SED and Equation (8) in Casey et al. (2011):

$$
M_{\text {dust }}=\frac{S_{v} D_{\mathrm{L}}^{2}}{\kappa_{v} B_{v}(T)} \propto \frac{D_{\mathrm{L}}^{2}}{\kappa_{v} v^{2}},
$$

where $S_{v}$ is the flux density at frequency $v, \kappa_{v}$ is the dust mass absorption coefficient at $v, B_{v}(T)$ is the Planck function at temperature $T$, and $D_{\mathrm{L}}$ is the luminosity distance. We adopted a dust absorption coefficient of $\kappa_{850}=0.15 \mathrm{~m}^{2} \mathrm{~kg}^{-1}$ (Weingartner \& Draine 2001; Dunne et al. 2003). We derived a mean dust temperature $M_{\text {dust }}=10^{7.3} M_{\odot}$ for the sample.

We note that dust temperatures and masses depend critically on a thorough understanding of the radiative transfer involved as well as the underlying geometry of the dust cloud. This dust temperature can be taken as a characteristic $T_{\text {dust }}$ for the system (measured from the peak of the SED). Clearly the physics of these galaxies is more complex, comprised of many dust reservoirs of different temperatures. Unfortunately, investigating this is beyond the current scope of observations and 


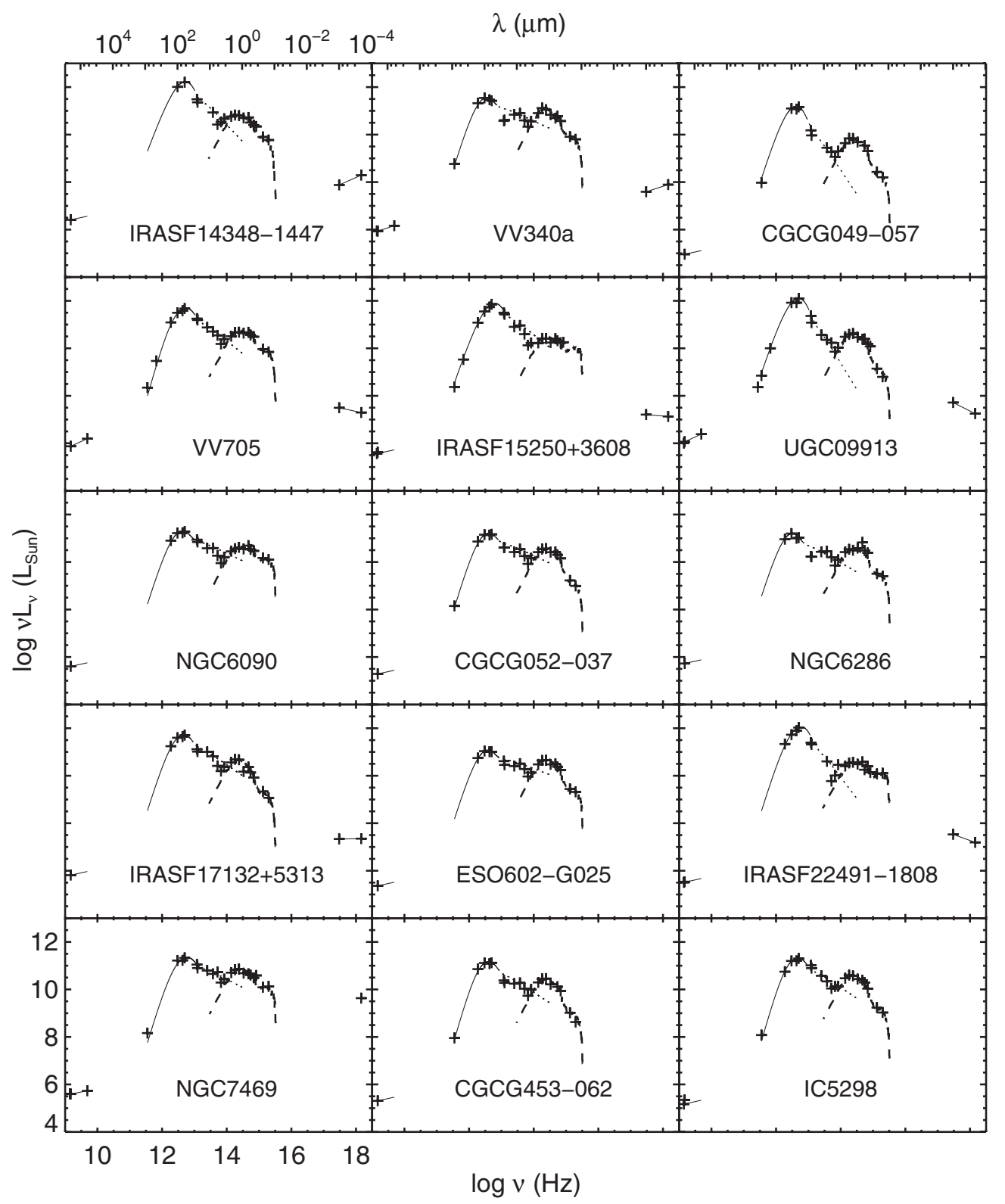

Figure 2. (Continued)

this work. We included our derivation of the dust temperature primarily as a baseline for comparison to the high- $z$ universe, though we caution that the physical interpretation of that quantity remains uncertain.

\subsubsection{Stellar Masses}

Stellar masses for the (U)LIRGs in our sample have been computed via two methods using two different IMFs, and the resulting masses are given in Table 10. Here, we discuss the differences among these measurements.

The two methods adopted for mass determination were to fit the UV-NIR part of the SEDs and to scale from the $H$-band luminosity. The $H$ band is usually selected for stellar mass conversion because it is at or near the photospheric peak of the stellar SED, and is thought not to be contaminated by hot dust emission from AGNs (Hainline et al. 2011). However, problems with $H$-band scaling may also arise due to thermally pulsing asymptotic giant branch stars for SEDs with a significant contribution from young stellar populations
(Walcher et al. 2011). On the other hand, the SED-fitted masses encompass the stellar component contributing to the optical peak, taking into account the treatment of dust with the designated dust extinction law. For the ensuing discussion, we focus on comparing the SED-fitted masses derived from using two different IMFs.

Our optical-NIR SED fitting procedure is based on stellar population synthesis models from Bruzual \& Charlot (2003) using 10 different broadband UV, optical, and infrared bands. Two different IMFs were used: a Chabrier IMF (Chabrier 2003) and a Salpeter IMF (Salpeter 1955). Since the IMF dictates the scaling of the mass-to-light $(M / L)$ ratio in converting luminosity to mass via its slope and mass cutoffs, we compare the masses derived from these two IMFs and assess their differences (Figure 9). The lower and upper mass cutoffs employed were $0.1 M_{\odot}$ and $100 M_{\odot}$, respectively (Bruzual \& Charlot 2003); no additional adjustments to the parameters of the IMFs have been made. The models assumed a star formation history with $\mathrm{SFH}=e^{-t / \tau}$, where $\tau$, varying 


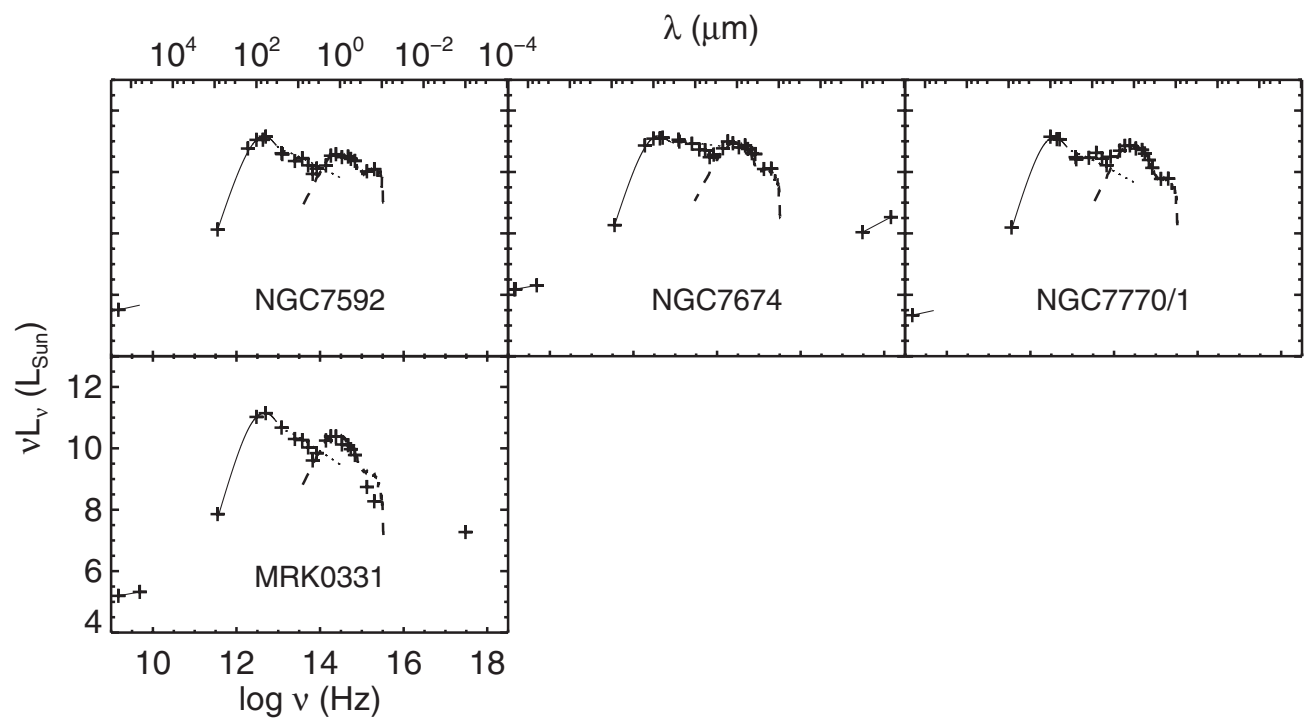

Figure 2. (Continued)

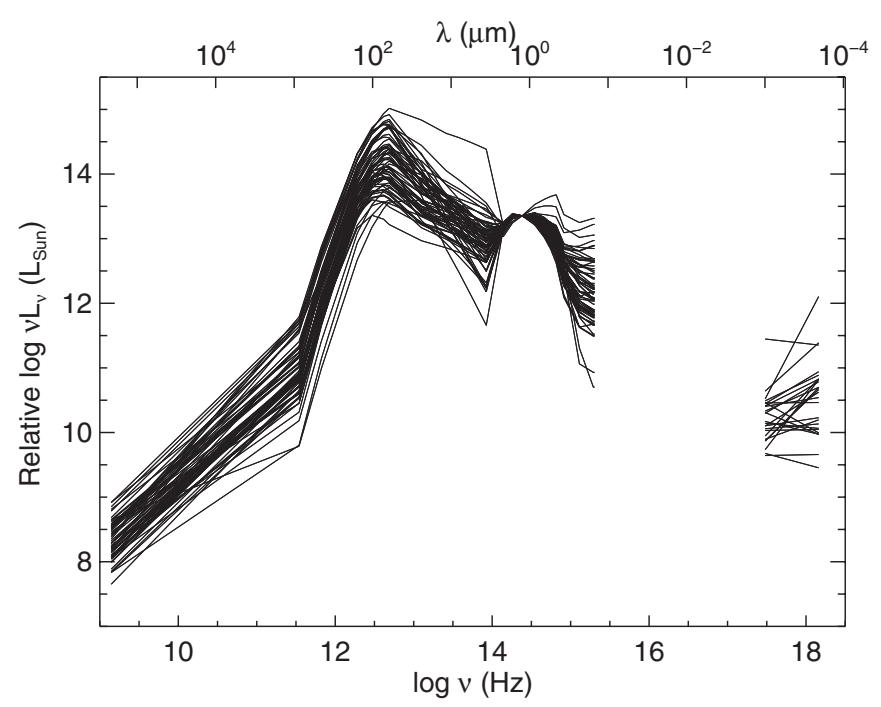

Figure 3. Full radio through X-ray SEDs normalized at $J$ band (near the peak of the stellar portion) for all 64 (U)LIRGs. The discrete data points represent photometry fitted at the 27 observed bands. Lines connecting the data points have been drawn to help guide the eye except for the regime between the far-UV and soft X-ray bands.

from $1-30$, is the $e$-folding parameter in years. The metallicity $(Z=0.004,0.008$, and 0.02$)$ has been treated as a free parameter as well.

In general, the mean difference between the masses derived from both methods is at the level of $0.26 \pm 0.41 \mathrm{dex}$, with the masses generally being underestimated from the Salpeter IMF. This was unexpected given that Salpeter IMF tends to result in higher stellar masses due to the difference in treatment of the low-mass end-the Chabrier IMF tends to be flatter and therefore more physical. Under the same input parameters the stellar models with Chabrier IMF fitted the UV light in our SEDs better than their Salpeter counterparts. We also note that the Chabrier IMF incorporates a more up-to-date treatment of UV radiation from young stars in starburst populations, and thus the Chabrier masses have been adopted as the stellar masses for the local (U)LIRGs.

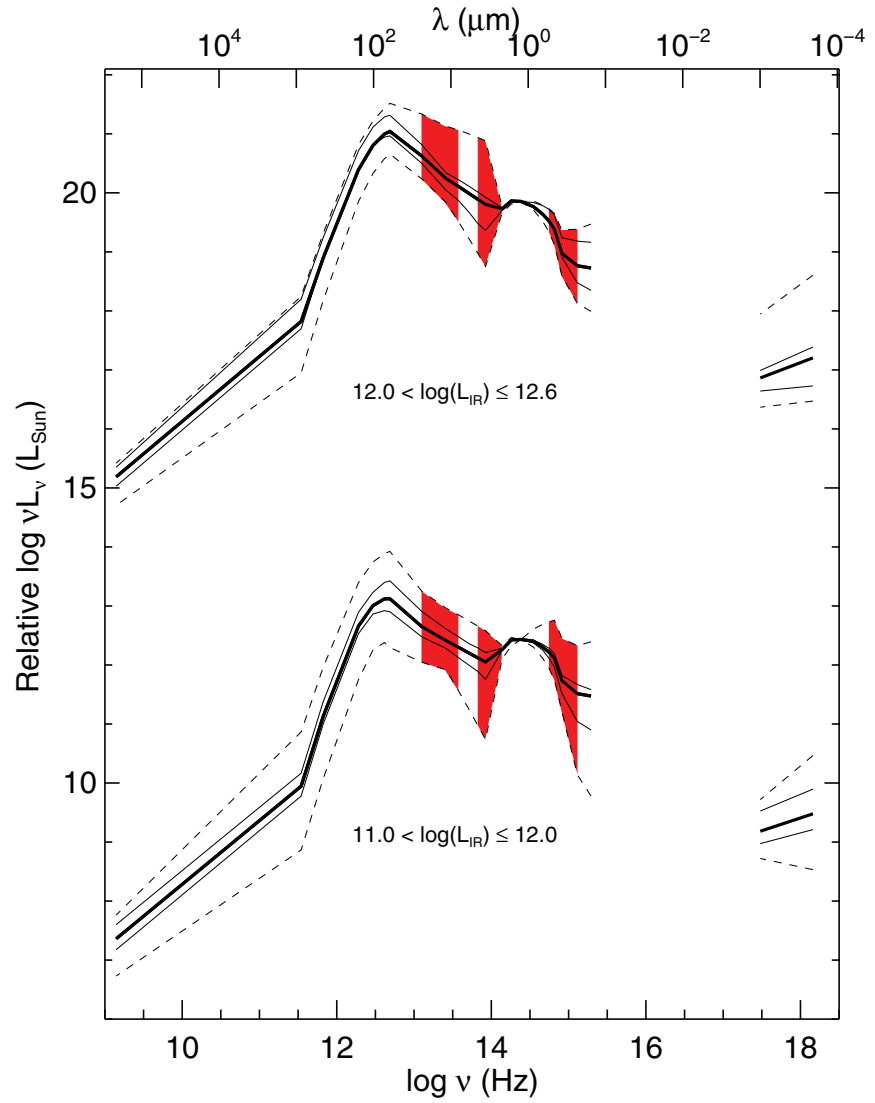

Figure 4. Mean radio through $\mathrm{X}$-ray SEDs normalized at $J$ band in two luminosity bins corresponding to ULIRGs (top) and LIRGs (bottom). The 50\% and $100 \%$ range for the SEDs are shown by the thin solid and dashed lines, respectively. The shaded regions mark the wavelength ranges used to calculate the three spectral indices, $\alpha_{2}^{1}$, as described in the text.

\subsubsection{Comparison of $M_{\star}$ and $L_{I R}$}

Figure 10 shows the (U)LIRGs as a distribution of SED-fitted mass in logarithmic scale. All but 3 of the 64 objects fall within $2 \sigma$ of the mean mass, $\log \left(M_{\star} / M_{\odot}\right)=10.79 \pm 0.40$.

For the individual subsamples of 53 LIRGs and 11 ULIRGs, we find the mean stellar masses to be $\log \left(M_{\star} / M_{\odot}\right)=10.75 \pm$ 


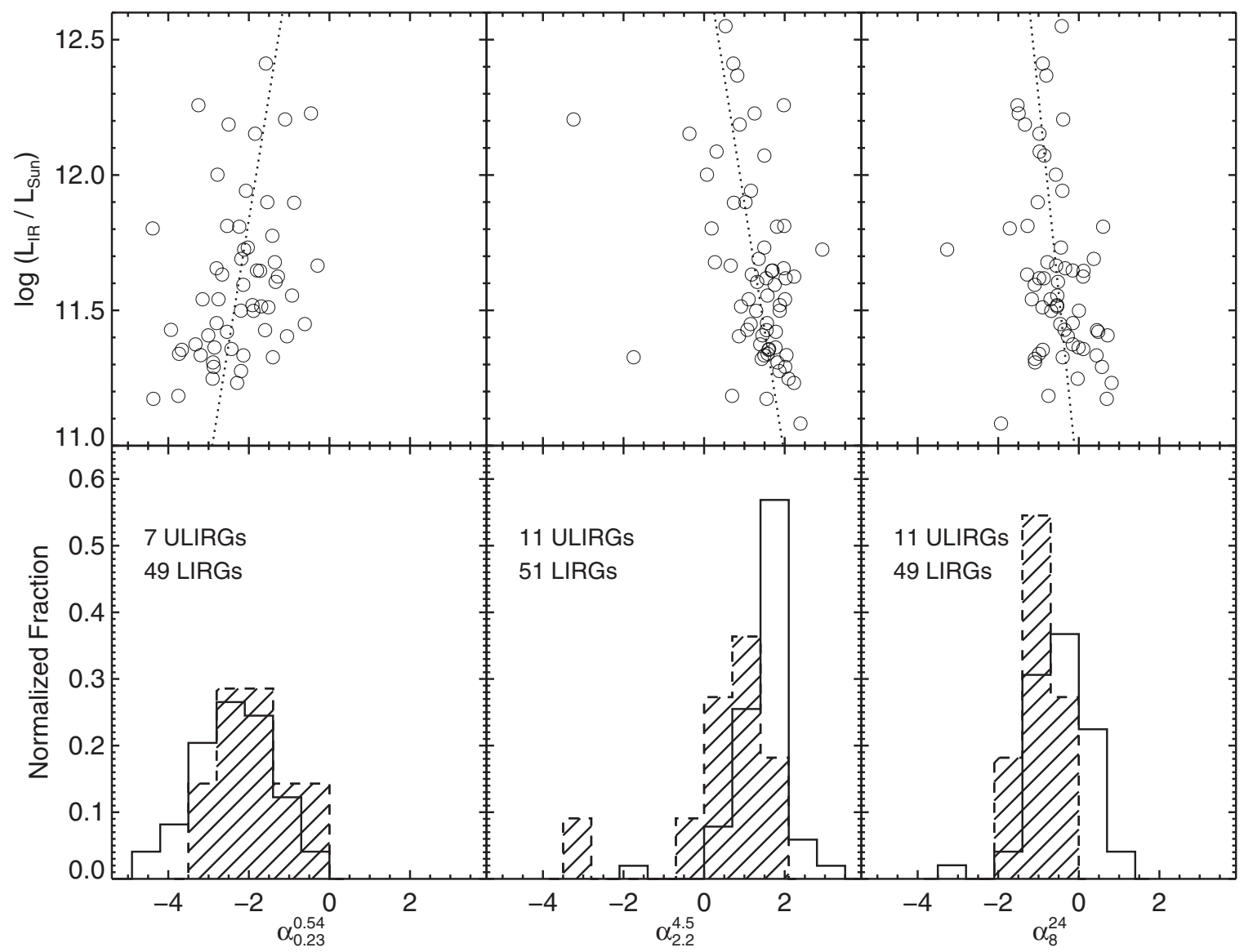

Figure 5. Spectral indices $\left(\alpha_{0.23}^{0.54}, \alpha_{2.2}^{4.5}\right.$, and $\left.\alpha_{8}^{24}\right)$ plotted against infrared luminosity (top), and their corresponding histograms (bottom). The best-fit (dotted) line within each of the top three panels shows the trends of the indices with increasing luminosity. The numbers of (U)LIRGs that are used to compute these indices (due to availability in the corresponding photometry data) are listed in the upper left-hand corner of the histograms. The hashed histograms represent ULIRGs, while the open histograms represent LIRGs.


Figure 6. Comparison of spectral indices $\left(\alpha_{0.23}^{0.54}, \alpha_{2.2}^{4.5}\right.$, and $\left.\alpha_{8}^{24}\right)$ for ULIRGs (hashed circles) and LIRGs (open circles). In the panels with the mid-IR index $\alpha_{8}^{24}$, the ULIRGs tend to cluster in a small region on the plot due to their very dusty nature, but the near-IR index $\alpha_{2.2}^{4.5}$ does not work as well in distinguishing the ULIRGs from their lower-luminosity counterparts.
0.39 for the LIRGs, and $\log \left(M_{\star} / M_{\odot}\right)=11.00 \pm 0.40$ for the ULIRGs. The factor of $\sim 2$ lower mean stellar mass for LIRGs is primarily due to a decreasing "low-mass tail" of objects with masses in the range $\log \left(M_{\star} / M_{\odot}\right)=9.3-10.3$. On the other extreme, the most massive object (UGC $08058=$ Mrk 231) appears to show hot dust emission from the central AGN, which contributes about $20 \%$ of the $H$-band flux (Surace \& Sanders 1999, 2000). Thus for this one object, we have corrected the fitted stellar mass accordingly.

Our results are consistent with estimates of the mass of objects of similar luminosity at higher redshift: $\log \left(M_{\mathrm{ULIRG}} / M_{\odot}\right) \sim$ $10.51 \pm 0.45$ (from Takagi et al. 2003, where the two-tailed unpaired $p$-value for the differences between our ULIRG masses gives 0.052 , which is not statistically significant); and log $\left(M_{\mathrm{LIRG}} / M_{\odot}\right) \sim 10.5$ (from Melbourne et al. 2008, who found that (U)LIRGs are more massive than "normal," non-LIRG galaxies that are morphologically irregular and spiral galaxies from the GOODS-S field, where $\left.\log \left(M_{\text {normal }} / M_{\odot}\right) \sim 9.5\right)$.

\subsubsection{Star Formation Rates}

Using the light contribution from the UV and IR, we determine the SFR for the unobscured and obscured stellar populations, respectively. The recipe from Wuyts et al. (2011) gives the following calibration for converting from infrared and 


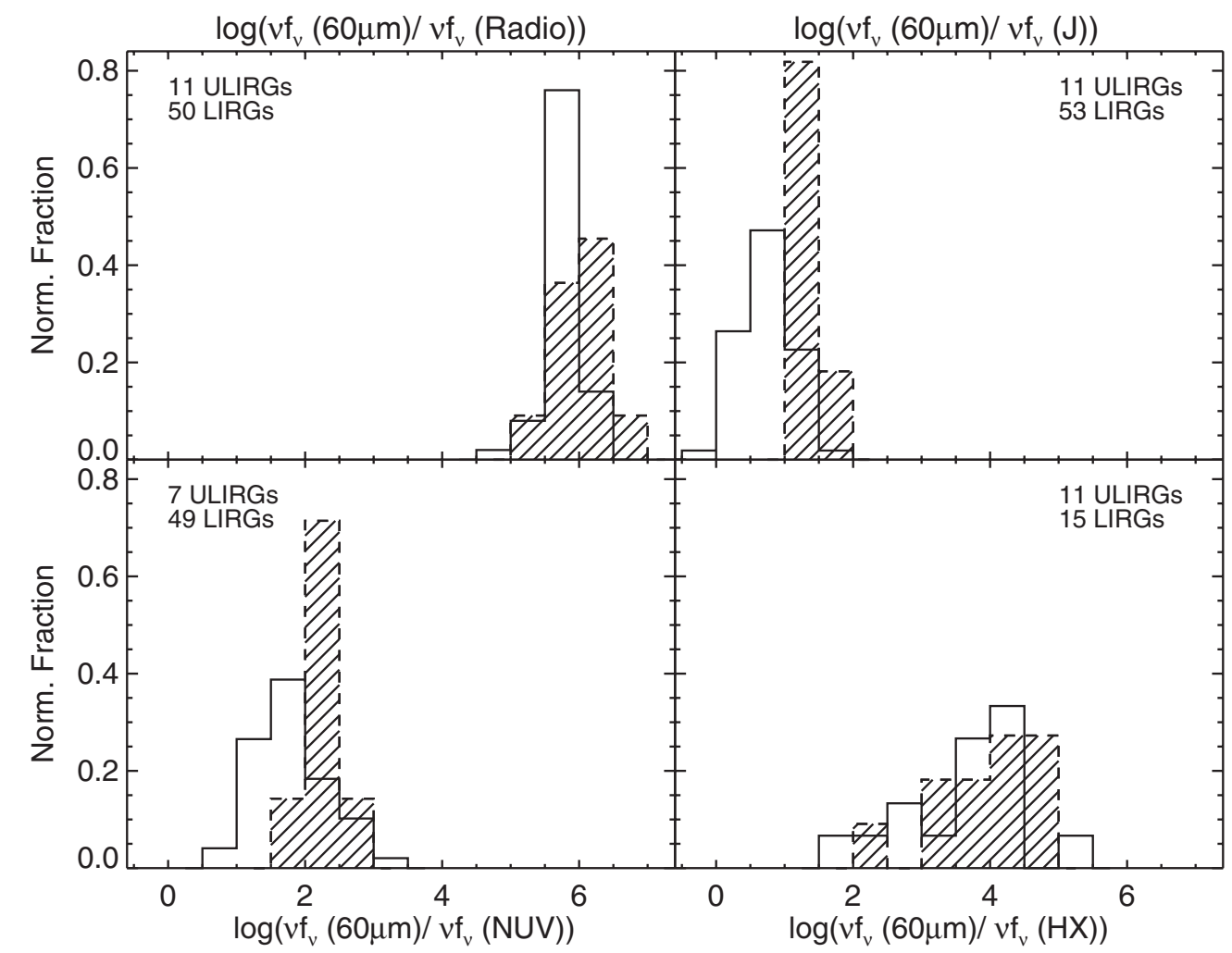

Figure 7. Normalized histograms of logarithmic ratios for $v f_{v}$ at $60 \mu \mathrm{m}$ to that at radio $(1.4 \mathrm{GHz}), J$ band $(1.2 \mu \mathrm{m}), \mathrm{NUV}(0.23 \mu \mathrm{m})$, and $\mathrm{HX}(2-10 \mathrm{keV})$. These histograms are normalized by the number of LIRGs (open histograms) and ULIRGs (hatched histograms) in each plot (as listed in the upper corners), as restricted by the availability of the photometry data used in these ratios.

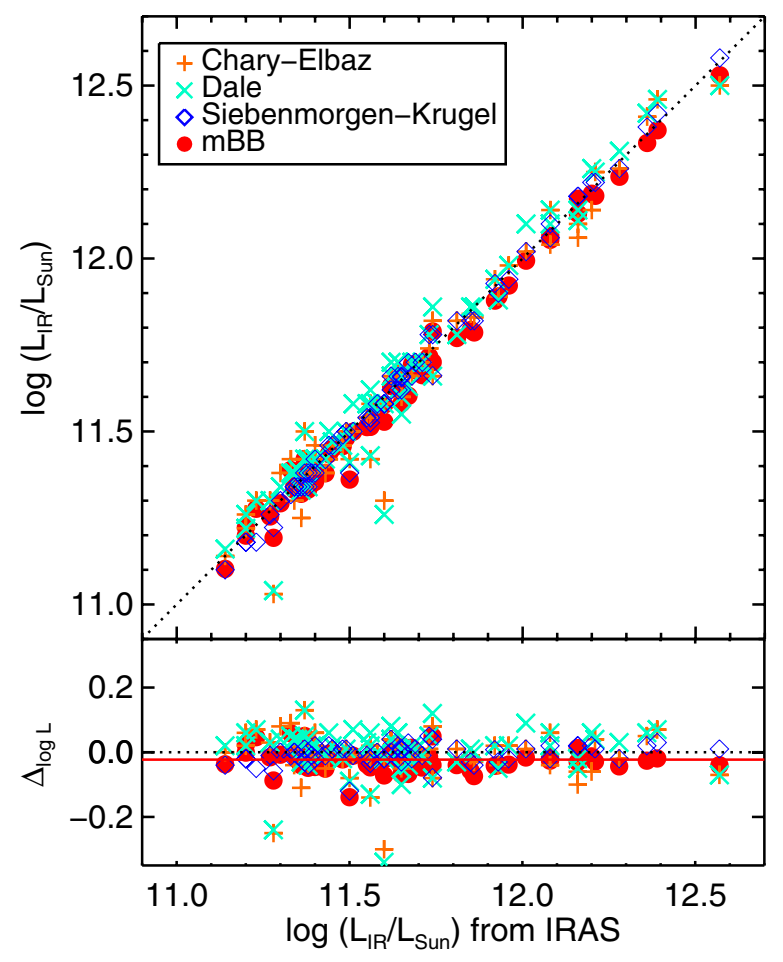

Figure 8. Comparison of the $L_{\mathrm{IR}}$ derived from IRAS fluxes and from fitting the FIR-submillimeter part of the SEDs for the (U)LIRGs (CE: orange cross, DH: cyan X, SK: blue open diamonds, and mBB: red filled circles). The dotted lines indicate linear correlation with zero offsets. As a one-to-one correspondence is expected, the residual $\Delta_{\log L} \equiv \log \left(L_{\mathrm{fit}} / L_{\odot}\right)-\log \left(L_{I R A S} / L_{\odot}\right)$ shows the scatter around the values predicted from IRAS. The red dash-dotted line shows that the mean of the mBB values is offset by 0.021 dex.

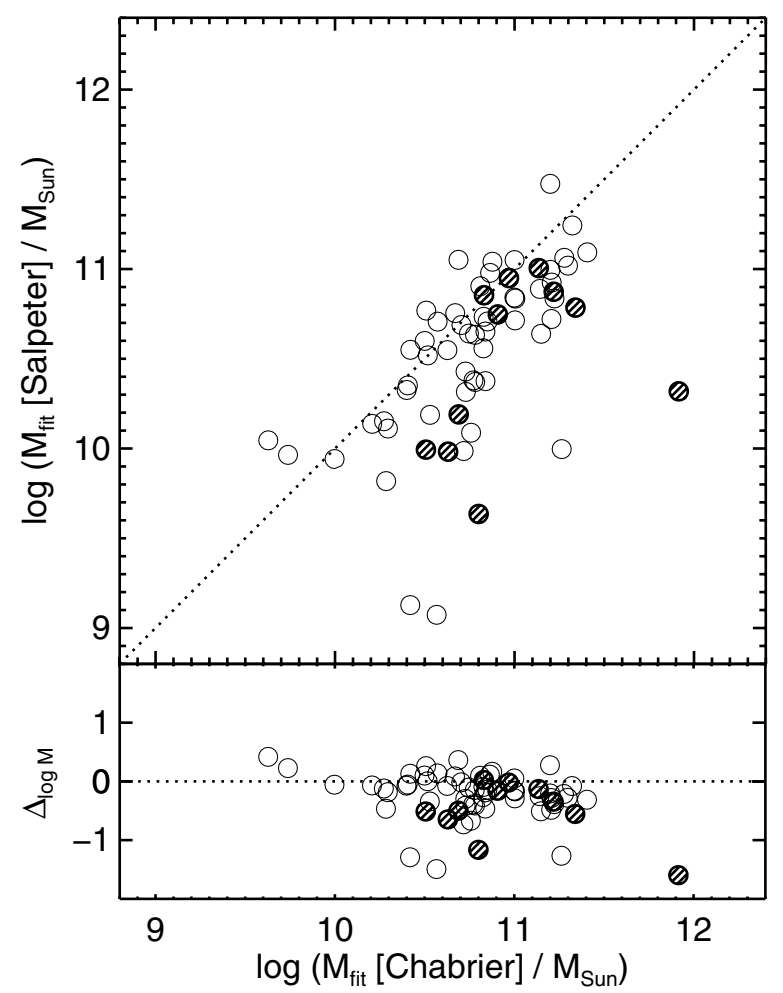

Figure 9. Comparison of the stellar mass estimates derived from fitting the UV-NIR part of the Salpeter-based BC03 SEDs and from that of the Chabrierbased ones. The residual plot illustrates that $\Delta_{\log \mathrm{M}} \equiv \log \left(M_{\mathrm{Sal}} / M_{\odot}\right)-$ $\log \left(M_{\text {Chab }} / M_{\odot}\right)$ centers at -0.26 , with the 25 th percentile at -0.41 and the 75 th percentile at -0.02 . The open circles are LIRGs and hatched circles are ULIRGs. The dotted lines indicate linear correlation with zero offset. 
Table 6

Mean SED Templates

\begin{tabular}{|c|c|c|c|c|}
\hline $\begin{array}{l}\log v \\
(\mathrm{~Hz})\end{array}$ & $\begin{array}{c}\lambda \\
(\mu \mathrm{m})\end{array}$ & $\begin{array}{c}\text { Band } \\
\text { (Name) }\end{array}$ & $\begin{array}{c}\log v L_{v} \\
\left(L_{\odot}\right) \\
\text { (ULIRGs) }\end{array}$ & $\begin{array}{c}\log v L_{v} \\
\quad\left(L_{\odot}\right) \\
(\text { LIRGs) }\end{array}$ \\
\hline 18.16 & 2.07E-04 & HX & 8.20 & 7.68 \\
\hline 17.48 & $9.93 \mathrm{E}-04$ & SX & 7.86 & 7.39 \\
\hline 15.29 & 0.15 & FUV & 9.72 & 9.68 \\
\hline 15.11 & 0.23 & NUV & 9.76 & 9.72 \\
\hline 14.91 & 0.37 & $U$ & 9.97 & 9.94 \\
\hline 14.82 & 0.46 & $B$ & 10.38 & 10.32 \\
\hline 14.75 & 0.54 & $V$ & 10.52 & 10.42 \\
\hline 14.67 & 0.65 & $R$ & 10.64 & 10.51 \\
\hline 14.54 & 0.86 & $I$ & 10.77 & 10.61 \\
\hline 14.38 & 1.24 & $J$ & 10.85 & 10.64 \\
\hline 14.26 & 1.65 & $H$ & 10.86 & 10.63 \\
\hline 14.14 & 2.17 & $K$ & 10.73 & 10.47 \\
\hline 13.93 & 3.56 & IRAC1 & 10.80 & 10.25 \\
\hline 13.82 & 4.51 & IRAC2 & 10.89 & 10.33 \\
\hline 13.72 & 5.76 & IRAC3 & 10.98 & 10.40 \\
\hline 13.58 & 7.96 & IRAC4 & 11.11 & 10.50 \\
\hline 13.41 & 11.60 & IRAS1 & 11.24 & 10.62 \\
\hline 13.10 & 23.84 & MIPS1 & 11.62 & 10.86 \\
\hline 13.10 & 23.88 & IRAS2 & 11.62 & 10.86 \\
\hline 12.69 & 61.49 & IRAS3 & 12.04 & 11.33 \\
\hline 12.62 & 72.56 & MIPS2 & 11.99 & 11.32 \\
\hline 12.47 & 101.98 & IRAS4 & 11.80 & 11.21 \\
\hline 12.28 & 156.96 & MIPS3 & 11.39 & 10.86 \\
\hline 11.82 & 449.57 & SCUBA1 & 9.89 & 9.33 \\
\hline 11.54 & 866.77 & SCUBA2 & 8.82 & 8.15 \\
\hline 9.15 & $2.14 \mathrm{E} 05$ & VLA & 6.19 & 5.57 \\
\hline
\end{tabular}

Table 7

Spectral Index versus $L_{\mathrm{IR}}$

\begin{tabular}{lcccr}
\hline \hline $\begin{array}{l}\lambda \text { range } \\
(\mu \mathrm{m})\end{array}$ & $\begin{array}{c}\alpha_{j}^{i} \\
\text { (index) }\end{array}$ & $\begin{array}{c}\bar{\alpha} \\
\text { (LIRGs) }\end{array}$ & $\begin{array}{c}\bar{\alpha} \\
\text { (ULIRGs) }\end{array}$ & $r$ \\
\hline $0.23-0.54$ & $\alpha_{0.23}^{0.54}$ & $-2.29 \pm 0.94$ & $-1.80 \pm 0.78$ & +0.32 \\
$2.2-4.5$ & $\alpha_{2.2}^{4.5}$ & $+1.49 \pm 0.70$ & $+0.84 \pm 0.69$ & -0.30 \\
$8.0-24.0$ & $\alpha_{8}^{24}$ & $-0.47 \pm 0.76$ & $-0.93 \pm 0.39$ & -0.38 \\
\hline
\end{tabular}

monochromatic UV luminosity at $2800 \AA$ to SFR:

$$
\mathrm{SFR}_{\mathrm{UV}+\mathrm{IR}}\left[M_{\odot} \mathrm{yr}^{-1}\right]=1.09 \times 10^{-10}\left(L_{\mathrm{IR}}+3.3 L_{2800}\right) / L_{\odot}
$$

Decomposing this quantity into separate UV and IR components, we consider the contribution to the total SFR from the $\mathrm{UV}$ and IR luminosities individually (Table 11). SFR $\mathrm{UV}_{\mathrm{UV}}$ ranges from $<1$ to $\sim 10 M_{\odot} \mathrm{yr}^{-1}$, while $\mathrm{SFR}_{\mathrm{IR}}$ is up to $\sim 50$ times larger. We show the fold enrichment of $S F R_{I R}$ to $S_{F R}$ as a function of infrared luminosity in Figure 11. The logarithmic difference for the LIRGs centered at $1.49 \pm 0.66 \mathrm{dex}$, while that for the ULIRGs is more clustered at $1.90 \pm 0.24$ dex. This figure highlights that while the infrared SFR in ULIRGs is $\sim 100$ times that determined from the UV, the fold enrichment in the LIRGs is only $\sim 30$ times with a large scatter potentially due to the large variations in dust geometry (i.e., single spirals undergoing minor merger events as opposed to major-merging pairs).

The mean $\log \mathrm{SFR}_{\mathrm{UV}+\mathrm{IR}}$ for the GOALS ULIRGs is $2.25 \pm$ 0.16 and that for the LIRGs is $1.57 \pm 0.19$. Corresponding specific SFRs (sSFR $=\mathrm{SFR}_{\mathrm{UV}+\mathrm{IR}} / M_{\star}$ ) have been computed and are also listed in Table 11. The effect on SFR from UV
Table 8

Variations in SEDs of (U)LIRGs

\begin{tabular}{|c|c|c|c|c|c|}
\hline $\begin{array}{l}\log v \\
(\mathrm{~Hz})\end{array}$ & $\begin{array}{c}\lambda \\
(\mu \mathrm{m})\end{array}$ & $\begin{array}{c}\text { Band } \\
\text { (Name) }\end{array}$ & $R$ & $\sigma_{R}$ & $\Delta R$ \\
\hline 18.16 & $2.07 \mathrm{E}-04$ & HX & -3.10 & 0.81 & 3.73 \\
\hline 17.48 & 9.93E-04 & SX & -3.69 & 0.54 & 2.24 \\
\hline 15.23 & 0.15 & FUV & -1.88 & 0.61 & 3.18 \\
\hline 15.11 & 0.23 & NUV & -1.83 & 0.56 & 2.88 \\
\hline 14.91 & 0.37 & $U$ & -1.62 & 0.48 & 1.70 \\
\hline 14.82 & 0.46 & $B$ & -1.23 & 0.47 & 1.68 \\
\hline 14.75 & 0.54 & V & -1.12 & 0.45 & 1.72 \\
\hline 14.67 & 0.65 & $R$ & -1.03 & 0.44 & 1.76 \\
\hline 14.54 & 0.86 & $I$ & -0.92 & 0.44 & 1.82 \\
\hline 14.38 & 1.24 & $J$ & -0.88 & 0.43 & 1.82 \\
\hline 14.26 & 1.65 & $H$ & -0.88 & 0.44 & 1.82 \\
\hline 14.14 & 2.17 & $K$ & -1.04 & 0.43 & 1.80 \\
\hline 13.93 & 3.56 & IRAC1 & -1.15 & 0.50 & 2.45 \\
\hline 13.82 & 4.51 & IRAC2 & -1.07 & 0.46 & 2.22 \\
\hline 13.72 & 5.76 & IRAC3 & -0.99 & 0.42 & 1.98 \\
\hline 13.58 & 7.96 & IRAC4 & -0.88 & 0.36 & 1.68 \\
\hline 13.41 & 11.60 & IRAS1 & -0.75 & 0.30 & 1.34 \\
\hline 13.10 & 23.84 & MIPS1 & -0.44 & 0.17 & 0.95 \\
\hline 13.10 & 23.88 & IRAS2 & -0.44 & 0.17 & 0.95 \\
\hline 12.69 & 61.49 & IRAS3 & 0.00 & 0.00 & 0.00 \\
\hline 12.62 & 72.56 & MIPS2 & -0.02 & 0.05 & 0.19 \\
\hline 12.47 & 101.98 & IRAS4 & -0.17 & 0.13 & 0.53 \\
\hline 12.28 & 156.96 & MIPS3 & -0.55 & 0.19 & 0.84 \\
\hline 11.82 & 449.57 & SCUBA1 & -2.07 & 0.25 & 1.16 \\
\hline 11.54 & 866.77 & SCUBA2 & -3.20 & 0.29 & 1.34 \\
\hline 9.15 & 2.14E05 & VLA & -5.81 & 0.28 & 1.75 \\
\hline
\end{tabular}

Notes. SEDs normalized at $60 \mu \mathrm{m}$ for the complete sample of 53 LIRGs and 11 ULIRGS. Band names correspond to the filters used in Tables 4 and $5 . R$ corresponds to the ratio $\log \left[\left(\nu L_{v}(\lambda)\right) /\left(\nu L_{v}(60 \mu \mathrm{m})\right)\right]$.

emission and trends with infrared luminosity as seen in the GOALS (U)LIRGs as well as the comparison to a nearby lower-luminosity sample (SINGS; Kennicutt et al. 2003) have been discussed extensively in Howell et al. (2010). Compared with the Howell et al. (2010) sample (with median sSFR = $3.9 \times 10^{-10} \mathrm{yr}^{-1}$ ), the median sSFR of our sample is $6.8 \times$ $10^{-10} \mathrm{yr}^{-1}$, or equivalent to a mass-doubling timescale of 1.5 Gyr. Our median sSFR value is higher because the GALEX sample is more complete at the lower-luminosity end (with less extinction by dust). The slope of the $\log \mathrm{SSFR}-\log M_{\star}$ relation is -0.78 (or 0.22 in $\log \mathrm{SFR}-\log M_{\star}$ space) for the (U)LIRGs, which is shallower than that reported for the high- $z$ infrared main-sequence galaxies (Rodighiero et al. 2011; Daddi et al. 2007).

\subsubsection{AGN Indicators}

Different wavelengths offer different methods for diagnosing AGN candidates; a multi-wavelength SED study allows simultaneous access to these various indicators and may be used to evaluate their effectiveness. We employ the radio-infrared flux ratio $(q)$ as defined by Condon et al. (1991) and the criteria specified by Yun et al. (2001; radio-excess: $q<1.64$; infraredexcess: $q>3.0$ ) as the basis of our comparison. Figure 12 shows $q$ plotted as a function of $L_{\mathrm{IR}}$ along with its distribution in the right panels. The mean $\langle q\rangle=2.41 \pm 0.29$ for the sample. With these limits, there are two LIRGs (with $L_{\mathrm{IR}}<11.6$ ) identified as radio-excess sources. These objects may be potential AGN hosts with a compact radio core or radio jets and lobes (Kartaltepe et al. 2010; Sanders \& Mirabel 1996). 


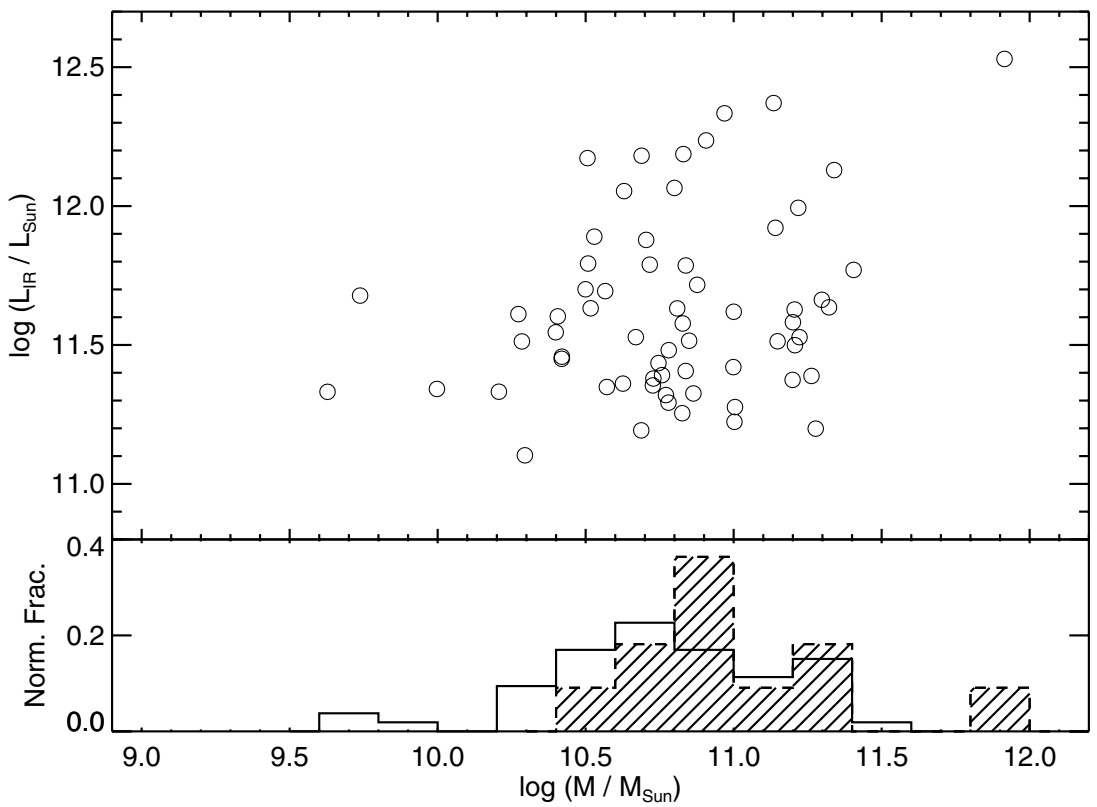

Figure 10. $\mathrm{mBB}$ infrared luminosity vs. stellar mass as derived from template fitting in logarithmic scale for our sample. The range of the masses spans more than a factor of 10 for the LIRGs. This is more clearly visualized in the histogram at the bottom, with bin width of 0.15 dex. The open histogram with solid line represents the LIRGs, while the hatched histogram represents the ULIRGs.

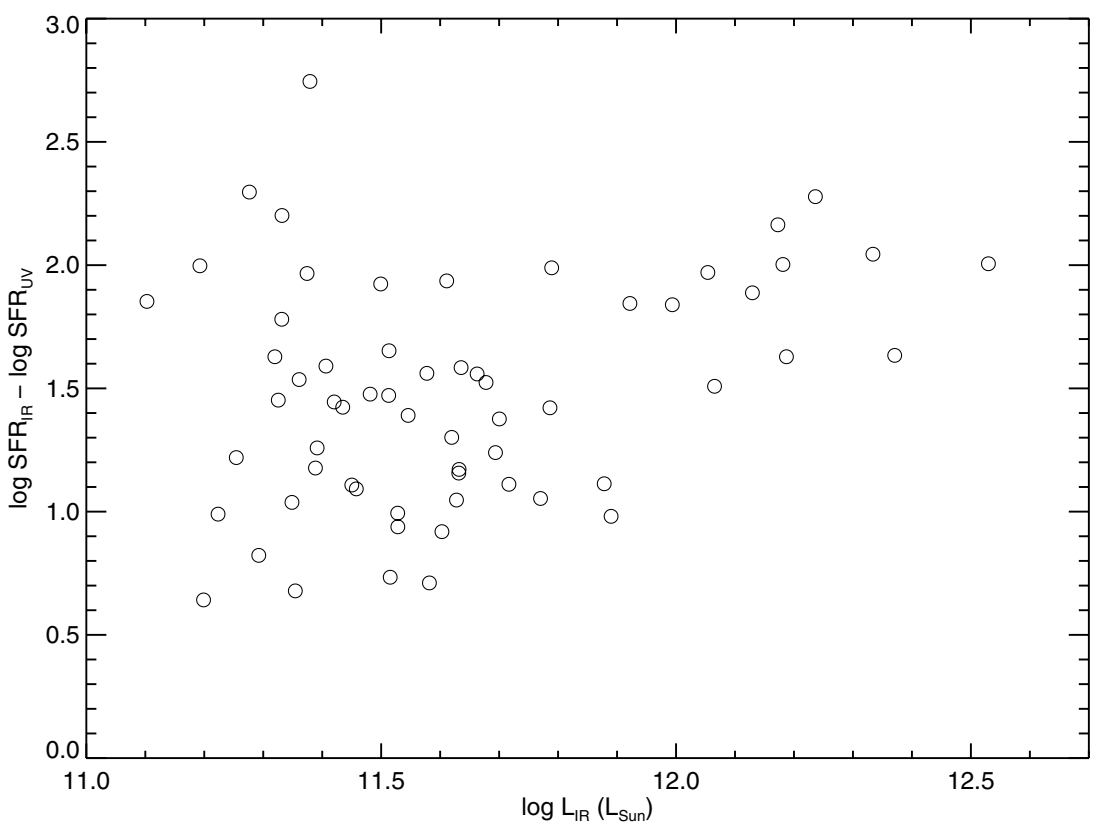

Figure 11. Logarithmic difference in SFR determined from the IR and the UV vs. $L_{\mathrm{IR}}$. The positive values in this difference reflect that the UV-derived SFR underpredict the SFR as measured from IR light.

On the other end, both of the infrared-excess sources are ULIRGs, which may be hosting dense and compact starbursts, or a dust-enshrouded AGN.

For objects with X-ray detection, we define two different criteria for identifying X-ray AGNs: (1) $L_{\mathrm{HX}}>10^{42} \mathrm{erg} \mathrm{s}^{-1}$ (Kartaltepe et al. 2010) and (2) hardness ratio, HR $>-0.3$ (Iwasawa et al. 2011). Seven systems qualified as an X-ray AGN by the first criterion, six by the second, and three of these objects were identified by both. Both radio-excess sources are very luminous in the $\mathrm{HX}$ band. All the HR $>-0.3 \mathrm{AGN}$ candidates are ULIRGs, though there may be a selection bias since all 11 of 11 ULIRGs in the GOALS sample have been observed and detected in the X-ray, but only 16 of 53 LIRGs have been observed thus far. Chandra observations of the lowerluminosity LIRGs have been proposed and awarded in Cycle 14 (PI: Sanders) to complete the sample.

Power-law SED provides a complementary way to select AGNs that might be heavily obscured and opaque to HX emission. We apply a criterion based on $\log \left(v L_{4.5}\right)-\log \left(v L_{2.2}\right)>0$ to select galaxies with power-law SED shape in the nearIR, corresponding to $F_{v}$ spectral slope of 0.4 (Kartaltepe et al. 2010; Alonso-Herrero et al. 2006; Donley et al. 2007). 


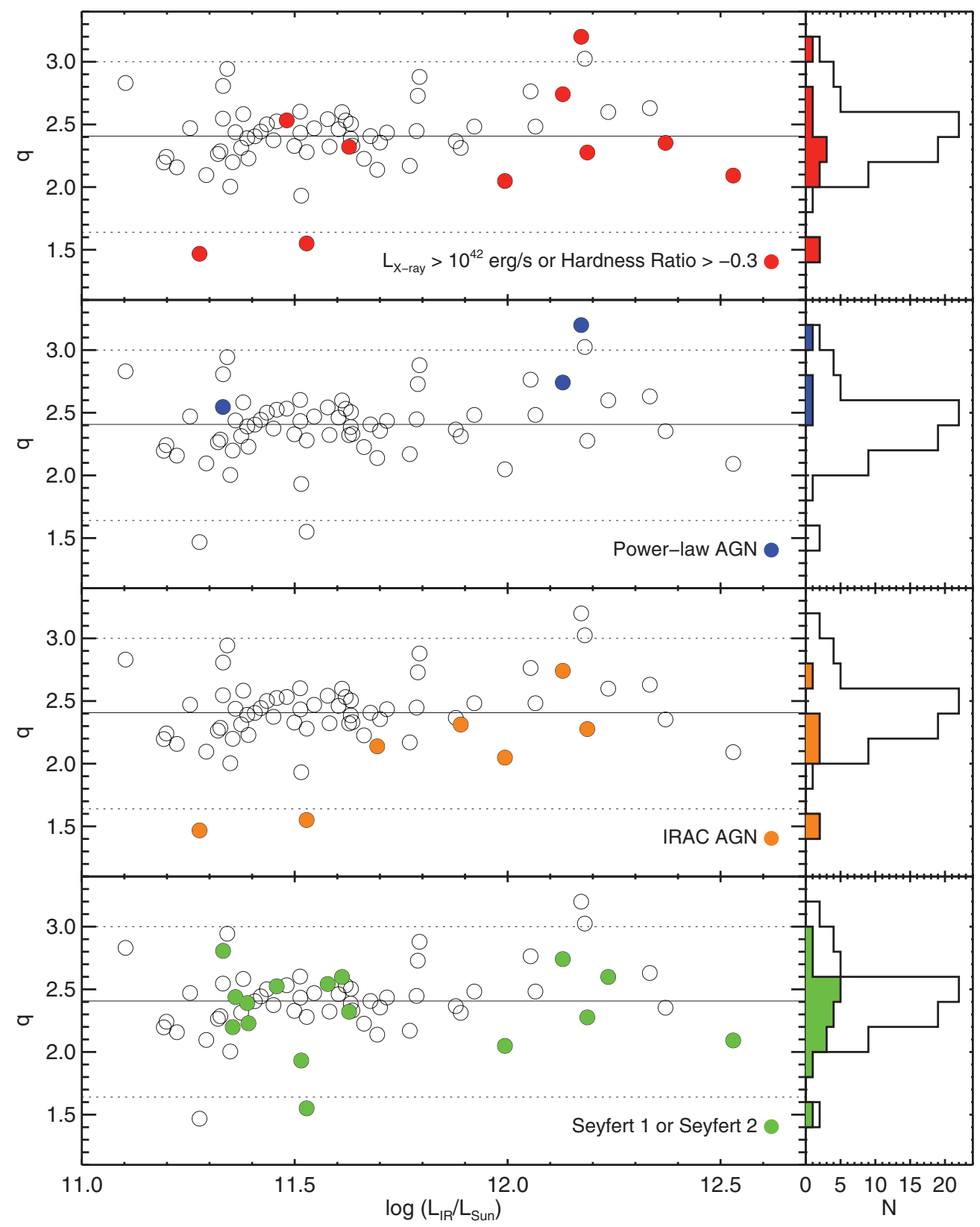

Figure 12. $q$ vs. $v L_{\mathrm{IR}}$ plots shown for the 64 (U)LIRGs along with the distribution in $q$ on the right panels. The solid line indicates the median value $(q=2.41)$ of the sample, with $\sigma=0.29$. The dotted lines indicate the radio-excess $(q<1.64)$ and infrared-excess $(q>3.0)$ criteria from Yun et al. (2001). From top to bottom are four different AGN indicators (filled circles/histograms): X-ray luminosity and hardness ratio, power-law slope, IRAC colors, and optical spectral classification.

Only three power-law AGN candidates are identified in the sample, and the two ULIRGs among these are both X-ray AGN candidates.

Additional MIR-based selection criteria have been devised to identify heavily obscured AGNs missed in deep X-ray surveys. In particular, selection based on IRAC color cuts (Lacy et al. 2004; Stern et al. 2005) is insensitive to obscuration but can trace AGN-heated dust, providing a powerful technique for discerning luminous obscured and unobscured AGNs. The revised IRAC color selection by Donley et al. (2012) is designed to incorporate the best aspects of the current IRAC wedges but to minimize star-forming contaminants and has been adopted here for selecting IRAC AGN candidates (see Equations (1) and (2) in Donley et al. 2012). This set of criteria results in seven IRAC AGN candidates in our sample, two of them being ULIRGs. Interestingly, most of the IRAC AGNs are below the median $q$ of the sample $\left(q_{\mathrm{med}}=2.41\right)$, with two of them being radio-excess sources.

Our last AGN indicator comparison involves selection based on optical emission line diagnostics. Yuan et al. (2010) applied a Sloan Digital Sky Survey based semiempirical optical spectral classification scheme to a large sample of local infrared galaxies, 57 of which are in our current sample. Among these, two are Seyfert 1 galaxies and 14 are Seyfert 2 systems. These Seyferts bear a distribution in $q$ similar to that of the entire sample. 
Table 9

Derived Infrared Luminosities, Dust Temperatures, and Dust Masses

\begin{tabular}{|c|c|c|c|}
\hline RBGS Name & $\begin{array}{c}\log \left(L_{\mathrm{IR}}\right)_{\mathrm{mBB}} \\
\left(L_{\odot}\right)\end{array}$ & $\begin{array}{c}T_{\text {dust }} \\
(K)\end{array}$ & $\begin{array}{c}\log \left(M_{\text {dust }}\right) \\
\left(M_{\odot}\right)\end{array}$ \\
\hline NGC 0034 & $11.48 \pm 0.01$ & $37.64 \pm 0.40$ & $6.88 \pm 0.06$ \\
\hline MCG-02-01-051/2 & $11.46 \pm 0.02$ & $32.70 \pm 0.57$ & $7.11 \pm 0.06$ \\
\hline NGC 0232 & $11.43 \pm 0.01$ & $28.35 \pm 0.18$ & $7.41 \pm 0.06$ \\
\hline $\mathrm{IC} 1623 \mathrm{~A} / \mathrm{B}$ & $11.66 \pm 0.01$ & $31.45 \pm 0.15$ & $7.42 \pm 0.06$ \\
\hline MCG-03-04-014 & $11.63 \pm 0.02$ & $31.09 \pm 0.50$ & $7.42 \pm 0.06$ \\
\hline CGCG436-030 & $11.68 \pm 0.02$ & $39.05 \pm 0.80$ & $7.14 \pm 0.06$ \\
\hline IRASF01364-1042 & $11.79 \pm 0.02$ & $35.37 \pm 0.65$ & $7.36 \pm 0.06$ \\
\hline IIIZw035 & $11.62 \pm 0.02$ & $35.86 \pm 0.65$ & $7.35 \pm 0.06$ \\
\hline NGC 0695 & $11.69 \pm 0.02$ & $27.90 \pm 0.36$ & $7.54 \pm 0.06$ \\
\hline NGC 0958 & $11.22 \pm 0.02$ & $23.42 \pm 0.32$ & $7.55 \pm 0.06$ \\
\hline UGC02238 & $11.39 \pm 0.01$ & $26.34 \pm 0.24$ & $7.13 \pm 0.06$ \\
\hline UGC02369 & $11.60 \pm 0.02$ & $34.01 \pm 0.52$ & $7.18 \pm 0.06$ \\
\hline IRASF03359+1523 & $11.51 \pm 0.03$ & $33.62 \pm 0.78$ & $7.12 \pm 0.06$ \\
\hline UGC02982 & $11.20 \pm 0.02$ & $27.33 \pm 0.45$ & $6.96 \pm 0.06$ \\
\hline ESO550-IG025 & $11.50 \pm 0.02$ & $28.95 \pm 0.36$ & $7.44 \pm 0.06$ \\
\hline NGC 1614 & $11.61 \pm 0.01$ & $42.23 \pm 0.15$ & $6.45 \pm 0.06$ \\
\hline IRASF05189-2524 & $12.13 \pm 0.01$ & $46.20 \pm 0.22$ & $8.02 \pm 0.06$ \\
\hline NGC 2623 & $11.58 \pm 0.02$ & $35.78 \pm 0.42$ & $7.35 \pm 0.06$ \\
\hline IRASF08572+3915 & $12.17 \pm 0.04$ & $46.09 \pm 1.12$ & $7.60 \pm 0.06$ \\
\hline UGC04881 & $11.70 \pm 0.02$ & $28.62 \pm 0.42$ & $7.43 \pm 0.06$ \\
\hline UGC05101 & $12.00 \pm 0.01$ & $28.75 \pm 0.28$ & $7.83 \pm 0.06$ \\
\hline MCG+08-18-013 & $11.33 \pm 0.02$ & $29.83 \pm 0.58$ & $6.86 \pm 0.06$ \\
\hline IC0563/4 & $11.28 \pm 0.01$ & $24.30 \pm 0.21$ & $7.57 \pm 0.06$ \\
\hline NGC 3110 & $11.41 \pm 0.01$ & $25.98 \pm 0.16$ & $7.26 \pm 0.06$ \\
\hline IRASF10173+0828 & $11.79 \pm 0.02$ & $36.78 \pm 0.72$ & $7.43 \pm 0.06$ \\
\hline IRASF10565+2448 & $12.05 \pm 0.02$ & $33.62 \pm 0.50$ & $7.54 \pm 0.06$ \\
\hline MCG+07-23-019 & $11.63 \pm 0.02$ & $29.35 \pm 0.42$ & $7.47 \pm 0.06$ \\
\hline CGCG011-076 & $11.38 \pm 0.03$ & $29.62 \pm 0.77$ & $7.18 \pm 0.06$ \\
\hline IC2810 & $11.64 \pm 0.02$ & $28.95 \pm 0.43$ & $7.56 \pm 0.06$ \\
\hline NGC 3690/IC694 & $11.89 \pm 0.01$ & $41.36 \pm 0.05$ & $7.09 \pm 0.06$ \\
\hline IRASF12112+0305 & $12.33 \pm 0.03$ & $35.78 \pm 0.75$ & $8.06 \pm 0.06$ \\
\hline IRASF12224-0624 & $11.32 \pm 0.03$ & $31.23 \pm 0.64$ & $7.32 \pm 0.06$ \\
\hline UGC08058 & $12.53 \pm 0.01$ & $45.67 \pm 0.13$ & $7.50 \pm 0.06$ \\
\hline NGC 4922 & $11.33 \pm 0.02$ & $37.81 \pm 0.67$ & $6.32 \pm 0.06$ \\
\hline ESO507-G070 & $11.53 \pm 0.01$ & $33.31 \pm 0.42$ & $7.19 \pm 0.06$ \\
\hline IC0860 & $11.10 \pm 0.02$ & $37.29 \pm 0.41$ & $6.84 \pm 0.06$ \\
\hline VV250a & $11.77 \pm 0.01$ & $37.81 \pm 0.31$ & $7.15 \pm 0.06$ \\
\hline UGC08387 & $11.72 \pm 0.01$ & $30.31 \pm 0.28$ & $7.28 \pm 0.06$ \\
\hline NGC 5104 & $11.25 \pm 0.01$ & $26.10 \pm 0.29$ & $7.03 \pm 0.06$ \\
\hline MCG-03-34-064 & $11.19 \pm 0.01$ & $53.90 \pm 0.64$ & $5.89 \pm 0.06$ \\
\hline NGC 5135 & $11.29 \pm 0.01$ & $27.26 \pm 0.15$ & $7.35 \pm 0.06$ \\
\hline NGC 5256 & $11.52 \pm 0.02$ & $31.45 \pm 0.59$ & $7.22 \pm 0.06$ \\
\hline NGC 5257/8 & $11.63 \pm 0.01$ & $27.14 \pm 0.23$ & $7.70 \pm 0.06$ \\
\hline UGC08696 & $12.18 \pm 0.01$ & $37.81 \pm 0.15$ & $7.57 \pm 0.06$ \\
\hline CGCG247-020 & $11.35 \pm 0.02$ & $31.16 \pm 0.66$ & $6.72 \pm 0.06$ \\
\hline IRASF14348-1447 & $12.37 \pm 0.35$ & $37.03 \pm 5.09$ & $8.35 \pm 0.09$ \\
\hline VV340a & $11.79 \pm 0.02$ & $25.80 \pm 0.28$ & $7.92 \pm 0.06$ \\
\hline CGCG049-057 & $11.33 \pm 0.01$ & $30.59 \pm 0.32$ & $7.23 \pm 0.06$ \\
\hline VV705 & $11.88 \pm 0.01$ & $37.03 \pm 0.38$ & $7.00 \pm 0.06$ \\
\hline IRASF15250+3608 & $12.07 \pm 0.02$ & $43.61 \pm 0.64$ & $7.49 \pm 0.06$ \\
\hline UGC09913 & $12.24 \pm 0.01$ & $35.69 \pm 0.10$ & $8.00 \pm 0.06$ \\
\hline NGC 6090 & $11.55 \pm 0.02$ & $30.95 \pm 0.37$ & $7.34 \pm 0.06$ \\
\hline CGCG052-037 & $11.45 \pm 0.02$ & $29.15 \pm 0.41$ & $7.22 \pm 0.06$ \\
\hline NGC 6286 & $11.42 \pm 0.01$ & $23.86 \pm 0.11$ & $7.81 \pm 0.06$ \\
\hline IRASF17132+5313 & $11.92 \pm 0.02$ & $33.01 \pm 0.53$ & $7.61 \pm 0.06$ \\
\hline ESO602-G025 & $11.34 \pm 0.01$ & $27.77 \pm 0.31$ & $7.37 \pm 0.06$ \\
\hline IRASF22491-1808 & $12.19 \pm 0.02$ & $40.79 \pm 0.88$ & $7.49 \pm 0.06$ \\
\hline NGC 7469 & $11.58 \pm 0.01$ & $39.96 \pm 0.32$ & $6.74 \pm 0.06$ \\
\hline CGCG453-062 & $11.37 \pm 0.02$ & $28.55 \pm 0.45$ & $7.01 \pm 0.06$ \\
\hline IC5298 & $11.53 \pm 0.01$ & $32.63 \pm 0.46$ & $6.88 \pm 0.06$ \\
\hline NGC 7592 & $11.39 \pm 0.02$ & $32.33 \pm 0.59$ & $7.21 \pm 0.06$ \\
\hline NGC 7674 & $11.51 \pm 0.03$ & $28.42 \pm 0.51$ & $7.44 \pm 0.06$ \\
\hline NGC 7771 & $11.35 \pm 0.03$ & $27.20 \pm 0.48$ & $7.28 \pm 0.06$ \\
\hline MRK0331 & $11.36 \pm 0.01$ & $32.70 \pm 0.41$ & $6.74 \pm 0.06$ \\
\hline
\end{tabular}

Table 10

Derived Stellar Masses

\begin{tabular}{|c|c|c|c|c|}
\hline $\begin{array}{l}\text { RBGS Name } \\
\text { (1) }\end{array}$ & $\begin{array}{c}\log \left(M_{\text {fit }}\right)_{\text {Sal }} \\
\left(M_{\odot}\right) \\
(2)\end{array}$ & $\begin{array}{c}\log \left(M_{\mathrm{H}}\right)_{\mathrm{Sal}} \\
\left(M_{\odot}\right) \\
(3)\end{array}$ & $\begin{array}{c}\log \left(M_{\mathrm{fit}}\right)_{\mathrm{Cha}} \\
\left(M_{\odot}\right) \\
(4)\end{array}$ & $\begin{array}{c}\log \left(M_{\mathrm{H}}\right)_{\text {Cha }} \\
\left(M_{\odot}\right) \\
(5)\end{array}$ \\
\hline NGC 0034 & 10.44 & 10.58 & 10.41 & 10.07 \\
\hline MCG-02-01-051/2 & 9.15 & 10.65 & 10.42 & 10.15 \\
\hline NGC 0232 & 10.72 & 10.84 & 11.05 & 10.34 \\
\hline IC1623A/B & 9.07 & 10.65 & 10.56 & 10.15 \\
\hline MCG-03-04-014 & 10.89 & 10.85 & 10.79 & 10.35 \\
\hline CGCG436-030 & 9.97 & 10.56 & 9.74 & 10.06 \\
\hline IRASF01364-1042 & 10.65 & 10.45 & 10.75 & 9.95 \\
\hline IIIZw035 & 10.15 & 10.25 & 10.26 & 9.75 \\
\hline NGC 0695 & 11.04 & 11.03 & 11.29 & 10.53 \\
\hline NGC 0958 & 11.05 & 11.14 & 11.27 & 10.63 \\
\hline UGC02238 & 10.36 & 10.55 & 10.74 & 10.04 \\
\hline UGC02369 & 11.19 & 11.03 & 11.29 & 10.53 \\
\hline IRASF03359+1523 & 10.73 & 10.29 & 11.09 & 9.79 \\
\hline UGC02982 & 11.07 & 10.54 & 10.76 & 10.04 \\
\hline ESO550-IG025 & 10.94 & 10.93 & 11.22 & 10.43 \\
\hline NGC 1614 & 10.51 & 10.63 & 10.51 & 10.13 \\
\hline IRASF05189-2524 & 10.97 & 10.91 & 11.36 & 10.41 \\
\hline NGC 2623 & 10.31 & 10.47 & 10.38 & 9.96 \\
\hline IRASF08572+3915 & 10.12 & 10.29 & 10.51 & 9.81 \\
\hline UGC04881 & 11.04 & 10.97 & 10.86 & 10.47 \\
\hline UGC05101 & 10.86 & 10.93 & 11.23 & 10.43 \\
\hline MCG+08-18-013 & 9.86 & 9.56 & 9.27 & 9.06 \\
\hline IC0563/4 & 10.72 & 10.87 & 10.95 & 10.36 \\
\hline NGC 3110 & 10.45 & 10.83 & 10.72 & 10.32 \\
\hline IRASF10173+0828 & 10.81 & 10.33 & 10.55 & 9.84 \\
\hline IRASF10565+2448 & 9.59 & 10.87 & 10.76 & 10.38 \\
\hline MCG+07-23-019 & 10.37 & 10.63 & 10.37 & 10.14 \\
\hline CGCG011-076 & 10.65 & 10.75 & 10.84 & 10.25 \\
\hline IC2810 & 11.05 & 10.99 & 11.01 & 10.49 \\
\hline NGC 3690/IC694 & 10.17 & 10.76 & 10.52 & 10.26 \\
\hline IRASF12112+0305 & 10.92 & 10.76 & 11.05 & 10.28 \\
\hline IRASF12224-0624 & 9.95 & 9.95 & 9.99 & 9.45 \\
\hline UGC08058 & 10.32 & 11.48 & 11.92 & 10.98 \\
\hline NGC 4922 & 11.48 & 10.97 & 11.19 & 10.47 \\
\hline ESO507-G070 & 10.64 & 10.78 & 11.15 & 10.28 \\
\hline IC0860 & 10.12 & 10.12 & 10.29 & 9.62 \\
\hline VV250a & 10.24 & 10.39 & 10.31 & 10.02 \\
\hline UGC08387 & 10.55 & 10.59 & 10.45 & 10.08 \\
\hline NGC 5104 & 10.56 & 10.81 & 10.83 & 10.29 \\
\hline MCG-03-34-064 & 10.84 & 10.75 & 11.01 & 10.25 \\
\hline NGC 5135 & 10.65 & 10.97 & 10.82 & 10.46 \\
\hline NGC 5256 & 10.72 & 11.01 & 10.85 & 10.51 \\
\hline NGC 5257/8 & 11.01 & 11.23 & 11.21 & 10.73 \\
\hline UGC08696 & 10.86 & 10.96 & 10.84 & 10.46 \\
\hline CGCG247-020 & 10.36 & 10.45 & 10.76 & 9.95 \\
\hline IRASF14348-1447 & 10.76 & 11.02 & 11.07 & 10.54 \\
\hline VV340a & 10.82 & 10.83 & 11.07 & 10.31 \\
\hline CGCG049-057 & 10.15 & 10.02 & 10.21 & 9.52 \\
\hline VV705 & 10.68 & 10.86 & 10.71 & 10.37 \\
\hline IRASF15250+3608 & 9.91 & 10.61 & 10.57 & 10.11 \\
\hline UGC09913 & 10.71 & 10.81 & 10.88 & 10.29 \\
\hline NGC 6090 & 10.75 & 10.73 & 10.66 & 10.23 \\
\hline CGCG052-037 & 10.65 & 10.72 & 10.75 & 10.25 \\
\hline NGC 6286 & 10.73 & 10.76 & 10.62 & 10.26 \\
\hline IRASF17132+5313 & 11.43 & 10.89 & 11.25 & 10.39 \\
\hline ESO602-G025 & 10.96 & 10.82 & 10.84 & 10.31 \\
\hline IRASF22491-1808 & 10.37 & 10.71 & 10.77 & 10.23 \\
\hline NGC 7469 & 11.02 & 11.05 & 10.85 & 10.54 \\
\hline CGCG453-062 & 10.55 & 10.62 & 10.63 & 10.12 \\
\hline IC5298 & 10.73 & 10.76 & 10.83 & 10.26 \\
\hline NGC 7592 & 10.07 & 10.73 & 10.75 & 10.23 \\
\hline NGC 7674 & 10.85 & 11.17 & 11.23 & 10.67 \\
\hline NGC 7770/1 & 10.02 & 11.08 & 11.28 & 10.57 \\
\hline MRK0331 & 10.21 & 9.67 & 10.19 & 9.88 \\
\hline
\end{tabular}

Notes. Column 2: Mass fit (UV-opt SED) with Salpeter IMF (Salpeter 1955). Column 3: Mass fit ( $H$ band) with Salpeter IMF (Salpeter 1955). Column 4: Mass fit (UV-opt SED) with Chabrier IMF (Chabrier 2003). Column 5: Mass fit ( $H$ band) with Chabrier IMF (Chabrier 2003). 
Table 11

SFR and sSFR for Local (U)LIRGs

\begin{tabular}{|c|c|c|c|c|}
\hline RBGS Name & $\begin{array}{r}\log \mathrm{SFR}_{\mathrm{UV}} \\
\left(M_{\odot} \mathrm{yr}^{-1}\right)\end{array}$ & $\begin{array}{l}\log \mathrm{SFR}_{\mathrm{IR}} \\
\left(M_{\odot} \mathrm{yr}^{-1}\right)\end{array}$ & $\begin{array}{c}\log \mathrm{SFR}_{\mathrm{UV}+\mathrm{IR}} \\
\left(M_{\odot} \mathrm{yr}^{-1}\right)\end{array}$ & $\begin{array}{c}\log \mathrm{sSFR}_{\mathrm{UV}+\mathrm{IR}} \\
\left(\mathrm{yr}^{-1}\right)\end{array}$ \\
\hline NGC 0034 & 0.40 & 1.50 & 1.54 & -8.88 \\
\hline MCG-02-01-051/2 & 0.38 & 1.50 & 1.53 & -8.89 \\
\hline NGC 0232 & 0.01 & 1.47 & 1.49 & -9.51 \\
\hline IC1623A/B & 0.49 & 1.72 & 1.74 & -8.82 \\
\hline MCG-03-04-014 & 0.51 & 1.68 & 1.71 & -9.10 \\
\hline CGCG436-030 & 0.19 & 1.71 & 1.72 & -8.02 \\
\hline IRASF01364-1042 & -0.16 & 1.80 & 1.81 & -8.91 \\
\hline IIIZw035 & -0.29 & 1.65 & 1.66 & -8.61 \\
\hline NGC 0695 & 0.14 & 1.69 & 1.71 & -9.59 \\
\hline NGC 0958 & 0.59 & 1.21 & 1.31 & -9.97 \\
\hline UGC02238 & -0.27 & 1.34 & 1.35 & -9.42 \\
\hline UGC02369 & 0.09 & 1.69 & 1.70 & -9.63 \\
\hline IRASF03359+1523 & 0.08 & 1.56 & 1.57 & -8.71 \\
\hline UGC02982 & -0.77 & 1.21 & 1.21 & -9.48 \\
\hline ESO550-IG025 & -0.39 & 1.54 & 1.54 & -9.67 \\
\hline NGC 1614 & 0.50 & 1.68 & 1.71 & -8.81 \\
\hline IRASF05189-2524 & 0.28 & 2.19 & 2.19 & -9.14 \\
\hline NGC 2623 & 0.19 & 1.60 & 1.62 & -8.78 \\
\hline IRASF08572+3915 & 0.05 & 2.21 & 2.21 & -8.29 \\
\hline UGC04881 & 0.64 & 1.74 & 1.77 & -9.10 \\
\hline UGC05101 & 0.19 & 2.02 & 2.03 & -9.19 \\
\hline MCG+08-18-013 & -0.41 & 1.35 & 1.36 & -8.27 \\
\hline IC0563/4 & 0.27 & 1.23 & 1.28 & -9.72 \\
\hline NGC 3110 & 0.71 & 1.38 & 1.47 & -9.26 \\
\hline IRASF10173+0828 & -2.27 & 1.87 & 1.87 & -8.64 \\
\hline IRASF10565+2448 & 0.59 & 2.11 & 2.12 & -8.68 \\
\hline MCG+07-23-019 & 0.72 & 1.64 & 1.69 & -8.71 \\
\hline CGCG011-076 & -0.15 & 1.46 & 1.47 & -9.37 \\
\hline IC2810 & 0.36 & 1.66 & 1.68 & -9.32 \\
\hline NGC 3690/IC694 & 0.95 & 1.95 & 1.99 & -8.54 \\
\hline IRASF12112+0305 & 0.33 & 2.35 & 2.36 & -8.61 \\
\hline IRASF12224-0624 & -2.44 & 1.37 & 1.37 & -8.63 \\
\hline UGC08058 & 0.56 & 2.58 & 2.58 & -9.33 \\
\hline NGC 4922 & -0.55 & 1.39 & 1.40 & -9.80 \\
\hline ESO507-G070 & -0.10 & 1.56 & 1.57 & -9.58 \\
\hline IC0860 & -0.71 & 1.11 & 1.12 & -9.18 \\
\hline VV250a & 0.40 & 1.79 & 1.81 & -9.03 \\
\hline UGC08387 & 0.36 & 1.73 & 1.75 & -8.75 \\
\hline NGC 5104 & 0.07 & 1.25 & 1.28 & -9.55 \\
\hline MCG-03-34-064 & -0.98 & 1.32 & 1.32 & -9.68 \\
\hline NGC 5135 & 0.51 & 1.33 & 1.39 & -9.39 \\
\hline NGC 5256 & 0.82 & 1.59 & 1.65 & -9.20 \\
\hline NGC 5257/8 & 0.91 & 1.63 & 1.71 & -9.49 \\
\hline UGC08696 & 0.60 & 2.21 & 2.22 & -8.61 \\
\hline CGCG247-020 & -1.33 & 1.39 & 1.40 & -9.33 \\
\hline IRASF14348-1447 & 0.77 & 2.39 & 2.40 & -8.73 \\
\hline VV340a & 0.75 & 1.77 & 1.81 & -9.60 \\
\hline CGCG049-057 & -0.83 & 1.36 & 1.36 & -8.85 \\
\hline VV705 & 0.80 & 1.92 & 1.95 & -8.75 \\
\hline IRASF15250+3608 & 0.12 & 2.09 & 2.09 & -8.54 \\
\hline UGC09913 & -0.01 & 2.26 & 2.27 & -8.64 \\
\hline NGC 6090 & 0.63 & 1.59 & 1.63 & -9.04 \\
\hline CGCG052-037 & 0.05 & 1.47 & 1.49 & -9.26 \\
\hline NGC 6286 & 0.35 & 1.38 & 1.42 & -9.15 \\
\hline IRASF17132+5313 & 0.11 & 1.97 & 1.98 & -9.16 \\
\hline ESO602-G025 & -0.09 & 1.35 & 1.36 & -9.50 \\
\hline IRASF22491-1808 & 0.22 & 2.21 & 2.22 & -8.47 \\
\hline NGC 7469 & 0.62 & 1.68 & 1.71 & -9.49 \\
\hline CGCG453-062 & -0.14 & 1.39 & 1.40 & -9.22 \\
\hline IC5298 & 0.05 & 1.62 & 1.63 & -9.20 \\
\hline NGC 7592 & 0.17 & 1.40 & 1.42 & -9.33 \\
\hline NGC 7674 & 0.57 & 1.59 & 1.63 & -9.60 \\
\hline NGC 7771 & 0.25 & 1.40 & 1.43 & -9.83 \\
\hline MRK0331 & 0.04 & 1.52 & 1.54 & -9.24 \\
\hline
\end{tabular}

\section{CONCLUSIONS}

We have used common aperture masks for the first time to assemble accurate radio through X-ray SEDs of a complete local sample of 53 LIRGs and 11 ULIRGs observable from the northern hemisphere $\left(\delta>-30^{\circ}\right.$ and $\left.|b|>30^{\circ}\right)$. We have utilized several new large data sets provided by GOALS's spaceand ground-based observations of (U)LIRGs along with additional archival data from the literature and our own previously unpublished ground-based optical-NIR data from Mauna Kea in our analysis of these SEDs. We summarize our findings as follows.

1. The SEDs for all objects are similar in that they show a broad, thermal stellar peak $(\sim 0.3-2 \mu \mathrm{m})$, and a dominant FIR $(\sim 40-200 \mu \mathrm{m})$ thermal dust peak, where $v f_{v}(60 \mu \mathrm{m}) / v f_{v}(V)$ varies from $\sim 2$ to 30 with increasing $L_{\mathrm{IR}}$. The logarithmic difference between the stellar and dust peaks is $1.2 \mathrm{dex}$ for the ULIRGs and $0.7 \mathrm{dex}$ for the LIRGs.

2. When normalized at IRAS $60 \mu \mathrm{m}$, the largest range in the luminosity ratio, $R(\lambda) \equiv \log \left[v L_{v}(\lambda) / v L_{v}(60 \mu \mathrm{m})\right]$, observed over the full sample is seen in the hard X-rays $(\mathrm{HX}=2-10 \mathrm{keV})$, where $\Delta R_{\mathrm{HX}}=3.73\left(\bar{R}_{\mathrm{HX}}=-3.10\right)$. A small range is found in the radio $(1.4 \mathrm{GHz}), \Delta R_{1.4 \mathrm{GHz}}=$ 1.75 , where the mean ratio is largest, $\left(\bar{R}_{1.4 \mathrm{GHz}}=-5.81\right)$.

3. Infrared luminosities, $\left.L_{\mathrm{IR}}(8-1000) \mu \mathrm{m}\right)$, have been recomputed using a modified blackbody fit (Casey et al. 2011) to the MIR-FIR-submillimeter SEDs. The new $L_{\text {IR }}$ values are overall $\sim 0.02$ dex lower than the original IRAS values (Sanders et al. 2003), primarily due to the disagreement with the color indices implied in the coefficients of the Perault (1987) equation. The simple, analytical blackbody fit results have thus been adopted in determining the FIR properties of the (U)LIRGs (e.g., $L_{\mathrm{IR}}, T_{\text {dust }}$, and $M_{\text {dust }}$ ).

4. The stellar masses computed using BC03 for LIRGs, $\log \left(M_{\text {LIRGs }} / M_{\odot}\right)=10.75 \pm 0.39$, and ULIRGs, $\log \left(M_{\text {ULIRGs }} / M_{\odot}\right)=11.00 \pm 0.40$, are consistent with mass estimates of higher redshift LIRGs from Melbourne et al. (2008) and with mass estimates of higher redshift ULIRGs from Takagi et al. (2003), respectively.

5. SFRs determined from infrared and monochromatic UV luminosities individually have been compared: $\mathrm{SFR}_{\mathrm{UV}}$ ranges from $<1$ to $\sim 10 M_{\odot} \mathrm{yr}^{-1}$, while $\mathrm{SFR}_{\mathrm{IR}}$ is up to $\sim 50$ times larger. The logarithmic difference for the ULIRGs is much more clustered $(1.90 \pm 0.24 \mathrm{dex})$ than for the LIRGs $(1.49 \pm 0.66 \mathrm{dex})$, plausibly due to large variations in dust geometry among the lower-luminosity objects.

6. Radio-infrared flux ratio $(q)$, along with other multiwavelength criteria, has been assessed as different AGN indicators. The results among the various selection techniques complement each other. About $60 \%$ of the ULIRGs (and 25\% of the LIRGs) would be classified as an AGN by at least one of the selection criteria.

V.U. thanks O. Ilbert and S. Arnouts for their help with using the Le PHARE code, C. J. Ma for his help with UH $2.2 \mathrm{~m}$ data acquisition and reduction, T.-T. Yuan for her help with various scientific and technical contributions, and C. W. K. Chiang for statistical consult and technical help with Figures 1 and 3. V.U. also extends appreciation toward the UH TAC for their generous support of this project in awarding telescope time on Mauna Kea, as well as Colin Aspin and the UH 2.2 m Telescope staff 
for their help and support in the acquisition of the groundbased optical photometry. This research has made use of the NASA/IPAC Extragalactic Database (NED) and IPAC Infrared Science Archive, which are operated by the Jet Propulsion Laboratory, California Institute of Technology, under contract with the National Aeronautics and Space Administration. This publication has also made use of data products from the Two Micron All Sky Survey, which is a joint project of the University of Massachusetts and the Infrared Processing and Analysis Center/California Institute of Technology, funded by the National Aeronautics and Space Administration and the National Science Foundation. V.U. wishes to acknowledge funding support from the NASA Harriet G. Jenkins Predoctoral Fellowship Project and Giovanni Fazio via the Smithsonian Astrophysical Observatory Predoctoral Fellowship and JPL Contract/IRAC GTO Grant 1256790. This paper is dedicated to the memory of Michele Dufault, who led the effort to obtain accurate large aperature photometric optical and near-infrared data for our complete sample of objects, and whose early work was critical in our decision to publish this paper.

\section{REFERENCES}

Alonso-Herrero, A., Pérez-González, P. G., Alexander, D. M., et al. 2006, ApJ, 640, 167

Armus, L., Mazzarella, J. M., Evans, A. S., et al. 2009, PASP, 121, 559

Bruzual, G., \& Charlot, S. 2003, MNRAS, 344, 1000

Calzetti, D., Kinney, A. L., \& Storchi-Bergmann, T. 1994, ApJ, 429, 582

Casey, C. M. 2012, MNRAS, 425, 3094

Casey, C. M., Chapman, S. C., Neri, R., et al. 2011, MNRAS, 415, 2723

Chabrier, G. 2003, PASP, 115, 763

Chary, R., \& Elbaz, D. 2001, ApJ, 556, 562

Condon, J. J., Helou, G., Sanders, D. B., \& Soifer, B. T. 1990, ApJS, 73,359

Condon, J. J., Helou, G., Sanders, D. B., \& Soifer, B. T. 1996, ApJS, 103, 81

Condon, J. J., Huang, Z., Yin, Q. F., \& Thuan, T. X. 1991, ApJ, 378, 65

Daddi, E., Dickinson, M., Morrison, G., et al. 2007, ApJ, 670, 156

Dale, D. A., \& Helou, G. 2002, ApJ, 576, 159

Donley, J. L., Koekemoer, A. M., Brusa, M., et al. 2012, ApJ, 748, 142
Donley, J. L., Rieke, G. H., Pérez-González, P. G., Rigby, J. R., \& AlonsoHerrero, A. 2007, ApJ, 660, 167

Dunne, L., Eales, S., Edmunds, M., et al. 2000, MNRAS, 315, 115

Dunne, L., Eales, S., Ivison, R., Morgan, H., \& Edmunds, M. 2003, Nature, 424, 285

Dunne, L., \& Eales, S. A. 2001, MNRAS, 327, 697

Hainline, L. J., Blain, A. W., Smail, I., et al. 2011, ApJ, 740, 96

Helou, G., Soifer, B. T., \& Rowan-Robinson, M. 1985, ApJ, 298, L7

Howell, J. H., Armus, L., Mazzarella, J. M., et al. 2010, ApJ, 715, 572

Ishida, C. M. 2004, PhD thesis, Univ. Hawaii, (Source DAI-B 65/04)

Iwasawa, K., Sanders, D. B., Teng, S. H., et al. 2011, A\&A, 529, A106

Jarrett, T. H., Chester, T., Cutri, R., Schneider, S. E., \& Huchra, J. P. 2003, AJ, 125,525

Kartaltepe, J. S., Sanders, D. B., Le Floc'h, E., et al. 2010, ApJ, 709, 572

Kennicutt, R. C., Jr., Armus, L., Bendo, G., et al. 2003, PASP, 115, 928

Komatsu, E., Dunkley, J., Nolta, M. R., et al. 2009, ApJS, 180, 330

Lacy, M., Storrie-Lombardi, L. J., Sajina, A., et al. 2004, ApJS, 154, 166

Landolt, A. U. 1983, AJ, 88, 439

Melbourne, J., Ammons, M., Wright, S. A., et al. 2008, AJ, 135, 1207

Perault, M. 1987, PhD thesis, Univ. Paris

Petric, A. O., Armus, L., Howell, J., et al. 2011, ApJ, 730, 28

Rodighiero, G., Daddi, E., Baronchelli, I., et al. 2011, ApJ, 739, L40

Salpeter, E. E. 1955, ApJ, 121, 161

Sanders, D. B., Mazzarella, J. M., Kim, D.-C., Surace, J. A., \& Soifer, B. T. 2003, AJ, 126, 1607

Sanders, D. B., \& Mirabel, I. F. 1996, ARA\&A, 34, 749

Sanders, D. B., Soifer, B. T., Elias, J. H., et al. 1988, ApJ, 325, 74

Siebenmorgen, R., \& Krügel, E. 2007, A\&A, 461, 445

Skrutskie, M. F., Cutri, R. M., Stiening, R., et al. 2006, AJ, 131, 1163

Soifer, B. T., Sanders, D. B., Madore, B. F., et al. 1987, ApJ, 320, 238

Stern, D., Eisenhardt, P., Goriian, V., et al. 2005, ApJ, 631, 163

Surace, J. A., \& Sanders, D. B. 1999, ApJ, 512, 162

Surace, J. A., \& Sanders, D. B. 2000, AJ, 120, 604

Surace, J. A., Sanders, D. B., \& Evans, A. S. 2000, ApJ, 529, 170

Surace, J. A., Sanders, D. B., Vacca, W. D., Veilleux, S., \& Mazzarella, J. M. 1998, ApJ, 492, 116

Takagi, T., Arimoto, N., \& Hanami, H. 2003, MNRAS, 340, 813

Vavilkin, T. 2011, PhD thesis, Stony Brook Univ. (Proquest Dissertations and Theses No. AAT 347781)

Walcher, J., Groves, B., Budavári, T., \& Dale, D. 2011, Ap\&SS, 331, 1

Weingartner, J. C., \& Draine, B. T. 2001, ApJ, 548, 296

Wuyts, S., Förster Schreiber, N. M., Lutz, D., et al. 2011, ApJ, 738, 106

Yuan, T.-T., Kewley, L. J., \& Sanders, D. B. 2010, ApJ, 709, 884

Yun, M. S., Reddy, N. A., \& Condon, J. J. 2001, ApJ, 554, 803 